Louisiana State University

LSU Digital Commons

LSU Master's Theses

Graduate School

$10-19-2018$

\title{
Liquid Jet Penetration and Breakup in a Free Supersonic Gas Jet
}

Hansen Jones

Louisiana State University and Agricultural and Mechanical College

Follow this and additional works at: https://digitalcommons.Isu.edu/gradschool_theses

Part of the Aerodynamics and Fluid Mechanics Commons, Other Mechanical Engineering Commons, and the Propulsion and Power Commons

\section{Recommended Citation}

Jones, Hansen, "Liquid Jet Penetration and Breakup in a Free Supersonic Gas Jet" (2018). LSU Master's Theses. 4817.

https://digitalcommons.Isu.edu/gradschool_theses/4817

This Thesis is brought to you for free and open access by the Graduate School at LSU Digital Commons. It has been accepted for inclusion in LSU Master's Theses by an authorized graduate school editor of LSU Digital Commons. For more information, please contact gradetd@lsu.edu. 
A Thesis

Submitted to the Graduate Faculty of the Louisiana State University and Agricultural and Mechanical College in partial fulfillment of the requirements for the degree of Master of Science

in

The Department of Mechanical and Industrial Engineering

by

Hansen Jones

B.S.M.E., Louisiana State University, 2016

December 2018 


\section{Acknowledgments}

First, I would like to thank my major professor Dr. Shyam Menon and the rest of my committee, Dr. Ingmar Schoegl and Dr. Keith Gonthier. Without their constant support and guidance this project could not have been possible. I would also like to thank Dr. Daniel Allgood from NASA Stennis Space Center for his collaborative efforts, he was a constant source of knowledge and his feedback and hard work getting simulation results was vital to the success of this work. When I arrived at the Energy and Propulsion Lab in late 2016 we had no physical lab space and only the vision of what could be done with the large amount of equipment now at our disposal. I'm very proud to have progressed from sweeping the floor and setting up desks in the lab to get it in operational shape to having a working experiment that has allowed for a lot of collaboration and exciting work to be completed and started. The constant hard work and handson attitude of Dr. Menon was indispensable in the success of this project and the lab as a whole.

I would also like to thank my lab mates, and more importantly my friends, who have been with me throughout the past two years in the lab: Chris Jeansonne, Wanjun Dang, Wei Zhao, Havi Rajora, and Samuel. A lab would be a very dull place indeed if not for a few friends to joke around with while you're there (or to hold a 30lb work piece while you attach it to a test stand) and I have been grateful to call them both co-workers and friends during my time here. I would also like to thank Girguis Sedky for his assistance in operating and setting up the Focusing Color Schlieren system.

I would lastly like to thank my family and friends. I wouldn't have returned to school had it not been for my grandparents suggesting (very "strongly suggesting") I return to school after moving back from Austin, TX and it's a decision I'm glad I made. I also wouldn't be here without the support of friends that got me through the few months of unemployment before returning to 
school, and supporting me more along the way to become the best engineer and champion Schlitz Pizza trivia player I could be. Most importantly though I have my parents to thank for supporting me the longest, and for instilling a good work ethic and sense of direction. I remember looking at the stars as a kid with them and have those moments to thank for me pursing my dream job. 


\section{Table of Contents}

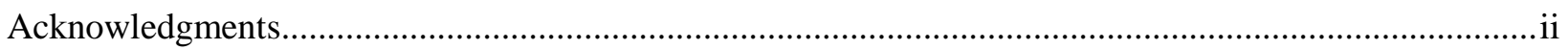

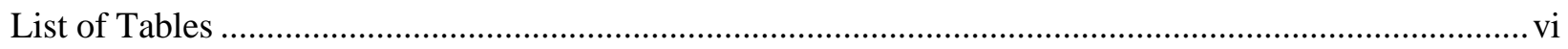

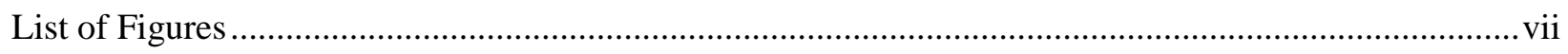

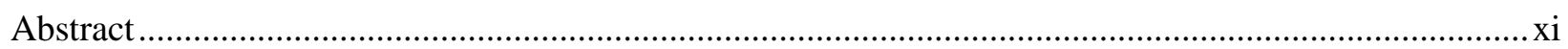

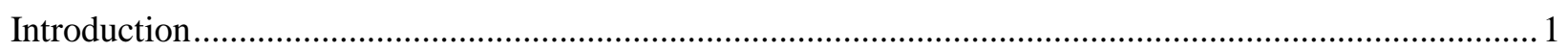

1.1. Motivation and problem statement …........................................................................... 1

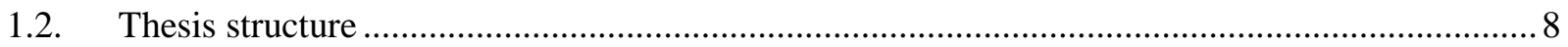

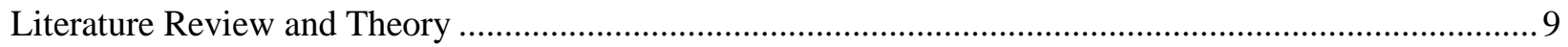

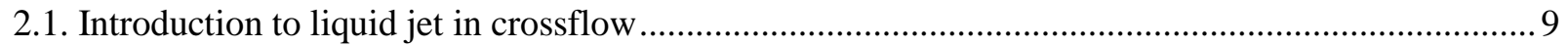

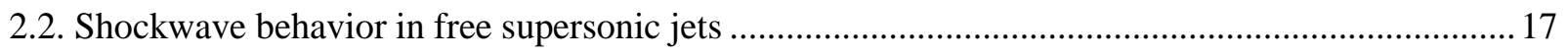

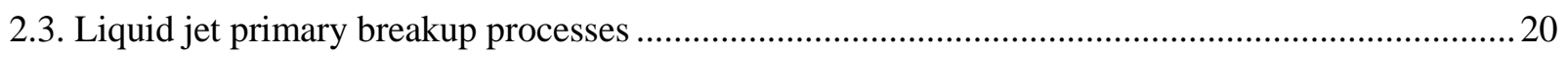

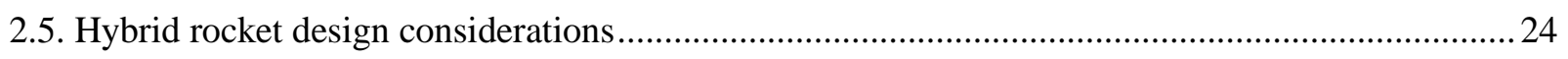

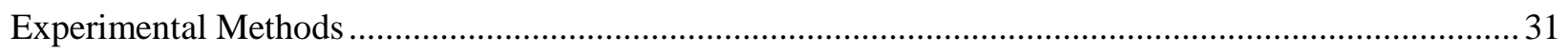

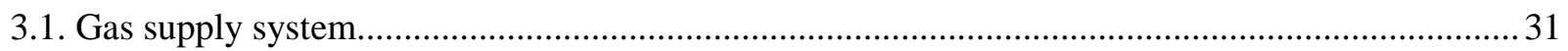

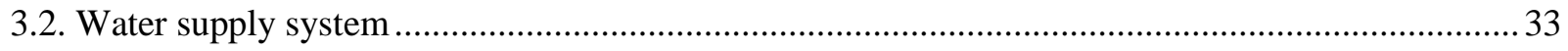

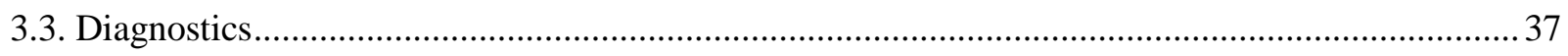

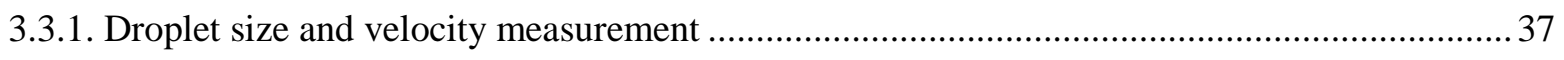

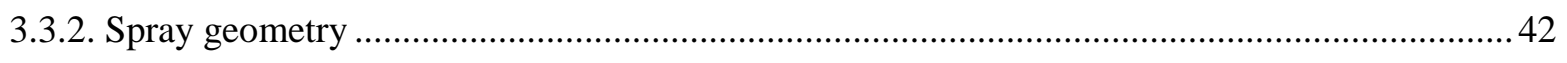

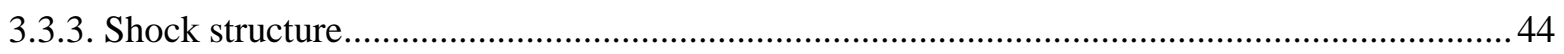

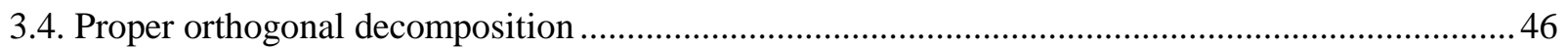

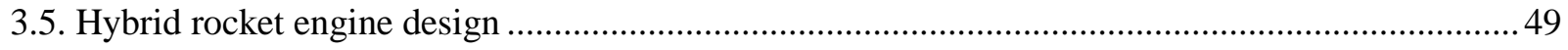

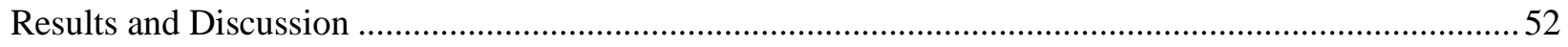

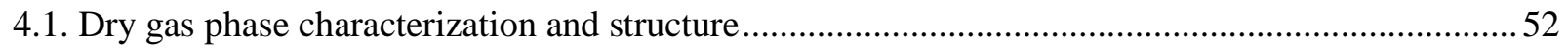

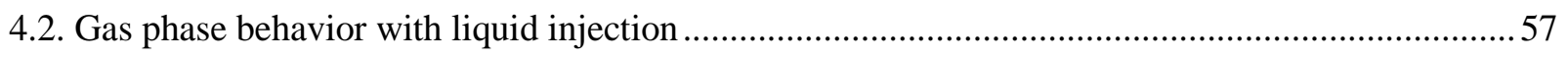

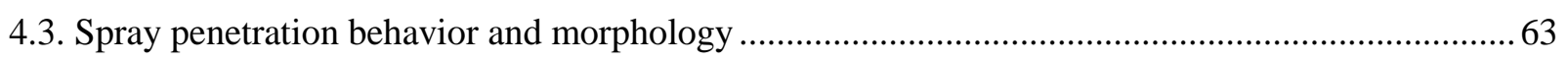

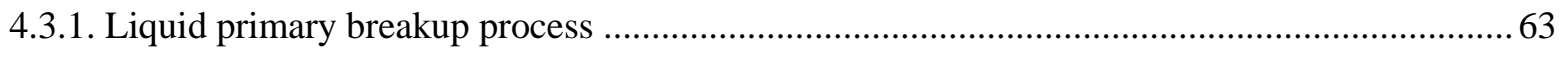

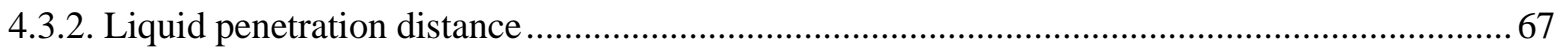

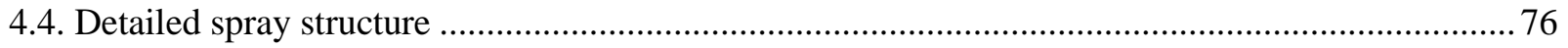

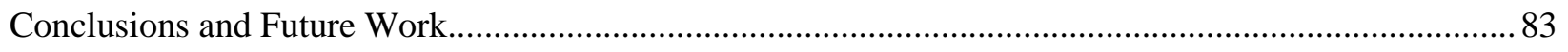

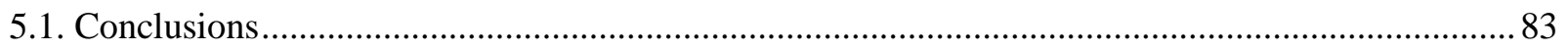

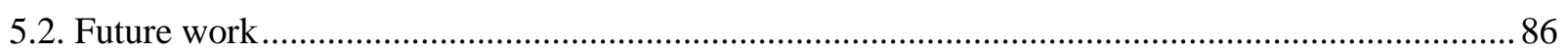




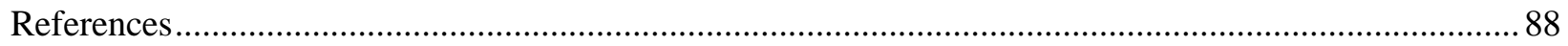

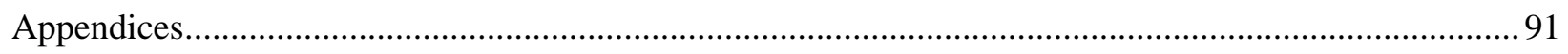

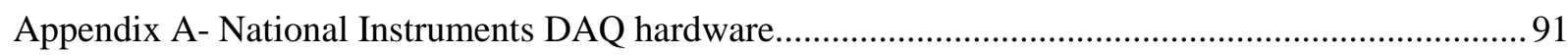

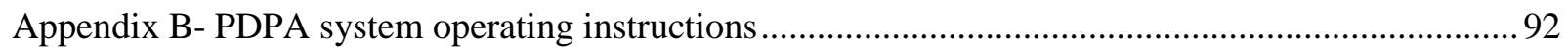

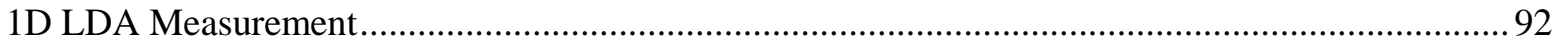

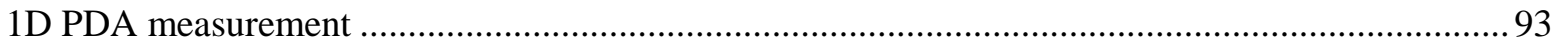

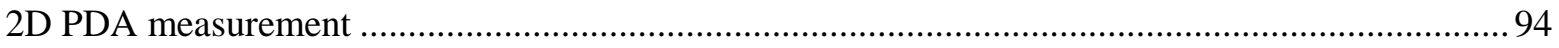

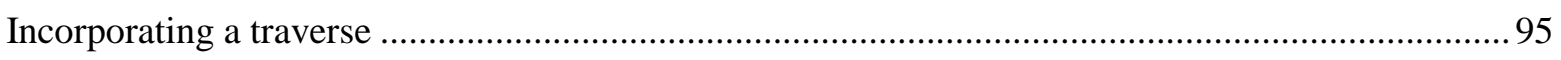

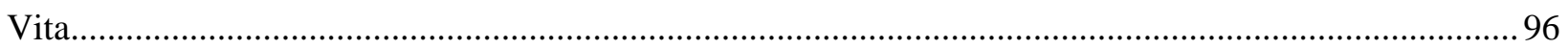




\section{List of Tables}

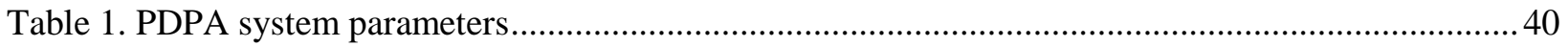

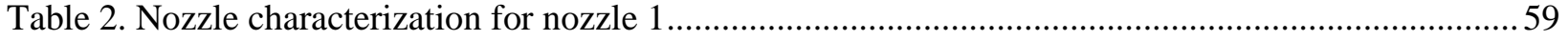

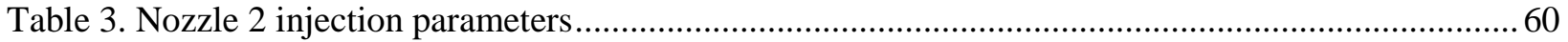

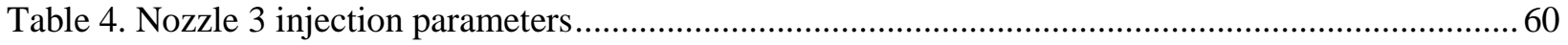

Table 5. Significant locations during PDPA measurements .................................................................. 77 


\section{List of Figures}

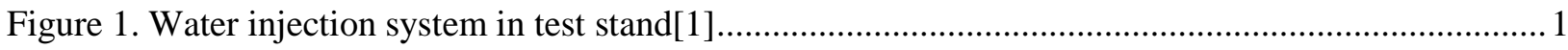

Figure 2: Left: Small scale test stand with spray ring[2], Right: Schematic showing deflector plate with water injection holes[3]....

Figure 3. Left: CFD results for gas phase, liquid phase, combined phases, Right: Results for cooled plate[3] 3

Figure 4. CFD results for Pressure (left), Temperature (middle), and Mach number (right)[4] 4

Figure 5. Left: Jet in crossflow[22], Right: Liquid penetration in a free jet[8] 6

Figure 6. General distinguishing features of the liquid jet breakup in crossflow [17] 11

Figure 7. Left: Upstream particle detection using holography, Right: Downstream particle detection using holography[21]. 13

Figure 8. Left: Velocity contour plot showing shockwave locations, Right: Numerical results compared with prior experimental results for various momentum ratios[22] .14

Figure 9. Left: Morphology for q=3, Right: Morphology for q=6[23] 15

Figure 10. Microjet injection into jet engine exhaust[25] 16

Figure 11. Top: Overexpanded gas jet shock structure, Bottom: Underexpanded jet shock structure [18] 19

Figure 12. Breakup process of the jet illustrating column and shear breakup[28]....

Figure 13. Initial breakup regime map for two fluid interfaces[28].

Figure 14. Left: Column breakup, Right: Surface breakup[28]

Figure 15. Hybrid rocket engine schematic [29] .25

Figure 16. Sample specific impulse as a function of o/f for HTPB fuel [31] .27 
Figure 17. Regression rate data for $\mathrm{O} 2$ hybrid engines for various solid fuels as a function of oxidizer mass flux [32]

Figure 18. Laboratory compressed air supply system. 31

Figure 19. Left: Dimensioned air nozzle drawing, Right: Air nozzle isometric view .32

Figure 20. Water pressurized storage cylinder. .33

Figure 21. Water nozzle flow rate as a function of back pressure .34

Figure 22. Left: fluid flow path diagram, Right: Experimental setup with compressed air and water nozzles

Figure 23. Screenshot of LabVIEW interface...... .36

Figure 24. Left: Compressed air inlet and chamber, Right: Vertically actuated water nozzle .37

Figure 25. Schematic of PDPA operation [35] .39

Figure 26. PDPA system and experimental setup .41

Figure 27. PDPA system on 2D traverse

Figure 28. Experimental setup for the laser sheet imaging .43

Figure 29. Light source orientation for high speed photography 44

Figure 30. Traditional z-type Schlieren setup [37] .45

Figure 31. Schematic diagram of focusing color schlieren system[35]..... .46

Figure 32. POD decomposition of a laminar jet at low Weber number. (a) Jet snapshot (b-d) First four orthogonal modes [38]

Figure 33. Hybrid rocket engine schematic diagram .50

Figure 34. Hybrid rocket combustion chamber cross-section. .51 
Figure 35. Long run-time compressed air supply characterization curves for static pressure (left) and temperature (right)

Figure 36. Left: Gas phase imaged using FCS, Right: Gas phase simulation results plotting density gradient magnitude (Source: Danny Allgood, NASA SSC)

Figure 37. Gas phase simulation results plotting Mach number iso-contours (Source: Danny Allgood, NASA SSC)

Figure 38. Left: Liquid back pressure for $\mathrm{q}=0.937$, Right: Air inlet static pressure for $\mathrm{q}=0.397$ .57

Figure 39. Left: Liquid static temperature for $\mathrm{q}=0.937$, Right: Air inlet static temperature for $\mathrm{q}=0.937 \ldots 57$

Figure 40. Shock structure behavior at varied injection locations

Figure 41. FCS of low momentum ratio liquid injection

Figure 42. Modified regime diagram showing cases of interest

Figure 43. High-speed video still images showing shear breakup regime, nozzle $1 \mathrm{q}=0.624 \ldots$

Figure 44. High-speed video still images showing surface stripping breakup regime nozzle $1 \mathrm{q}=2.497 \ldots 65$

Figure 45. Snapshot, and 0th, 2nd, 3rd, 4th, and 5th orthogonal modes for $\mathrm{q}=0.624$ .66

Figure 46. Snapshot, and 0th, 2nd, 3rd, 4th, and 5th orthogonal modes for $\mathrm{q}=0.624$ .66

Figure 47. Spray edge detection process .68

Figure 48. Left: Edge location tracking and average for Nozzle $1 \mathrm{q}=0.62437$, Right: Edge location tracking and average for Nozzle $3 \mathrm{q}=2.497$.

Figure 49. Global penetration data for all nozzles at selected momentum ratio .70

Figure 50. Nozzle 1 global penetration .71

Figure 51. Nozzle 1 air jet penetration .72 
Figure 52. Nozzle 2 global penetration

Figure 53. Nozzle 2 air jet penetration. .73

Figure 54. Nozzle 3 global penetration. .73

Figure 55. Nozzle 3 air jet penetration. .74

Figure 56. Liquid penetration in projected gas jet location for $\mathrm{q}=0.624$ compared with correlation from[19]

Figure 57. Centerline PDPA measurement locations(Source: Danny Allgood, NASA SSC) .77

Figure 58. Histograms showing droplet velocity distributions at various injection pressures and locations downstream of the injection location.... .78

Figure 59. Histograms showing droplet diameter distributions at various injection pressures and locations downstream of the injection location.

Figure 60. Left: Simulation results for gas phase velocity magnitude, Right: Simulation results for gas phase axial velocity (Source: Danny Allgood, NASA SSC)

Figure 61. National Instruments DAQ hardware. 


\section{Abstract}

In the testing of today's rocket engines, both on large scale vertical test stands and smaller subscale horizontal component testing stands, it is extremely important to be able to accurately quantify and mitigate the thermal and acoustic loads the engines will generate on test stand infrastructure. Due to the large number of parameters that must be considered for many cases, development of a multi-phase computational code is under way to properly analyze and design water spray cooling systems used at NASA's Stennis Space Center (SSC) and across other NASA centers. As such, a small-scale experiment has been conducted at Louisiana State University to provide experimental results which can be used to inform the development and verify the validity of such a code, and allow for several important physical characteristics of liquid breakup phenomena to be examined. The interactions of free jet of compressed air and varied coherent liquid jet injection parameters and nozzle sizes are examined and compared to the traditional problem of jet in cross flow (JICF). Non-intrusive diagnostic tools are used to examine the behavior of the internal shockwave structure in the overexpanded gas jet with and without liquid injection and significant changes are seen for varied injection location not seen for traditional JICF. An extension of the regime map for primary liquid breakup is made and high-speed imaging shows that for varied injection pressure the primary breakup regime of the liquid jet is similar to what is expected from literature. As the liquid jet is able to influence the momentum of the gas jet, an examination of the average spray boundary location and droplet size measurements in the secondary breakup region show significant flow turning of the gas phase and a strong dependence on the relative size of each fluid jet not accounted for in traditional JICF. Progress towards implementation of a hybrid rocket engine is also presented as next steps for better matching test conditions at SSC. 


\section{Introduction}

\subsection{Motivation and problem statement}

In the development of today's rocket engines, as with any piece of mission critical hardware, it is extremely important to rigorously test at all stages of development to ensure the hardware is operating safely and within the design conditions. As such, the North American Space Administration (NASA) makes use of its John C. Stennis Space Center (SSC) for the testing of both hardware for its commercial partners including SpaceX and Blue Origin as well as its own hardware. Originally built in the 1960s for testing of Saturn V's massive F1 engines, the facility has since grown to accommodate testing for the RS-25 engines that will power NASA's upcoming Space Launch System (SLS) vehicle as well as subscale component testing for its commercial partners. In either case, the ground support equipment (GSE) necessary to conduct the tests in a safe manner is routinely subjected to high temperatures exceeding $3000 \mathrm{~K}$ and acoustic loading of over $150 \mathrm{~dB}$. In order to mitigate the risk of damage to test structure and personnel, water suppression systems like the one shown in Figure 1 below are used.

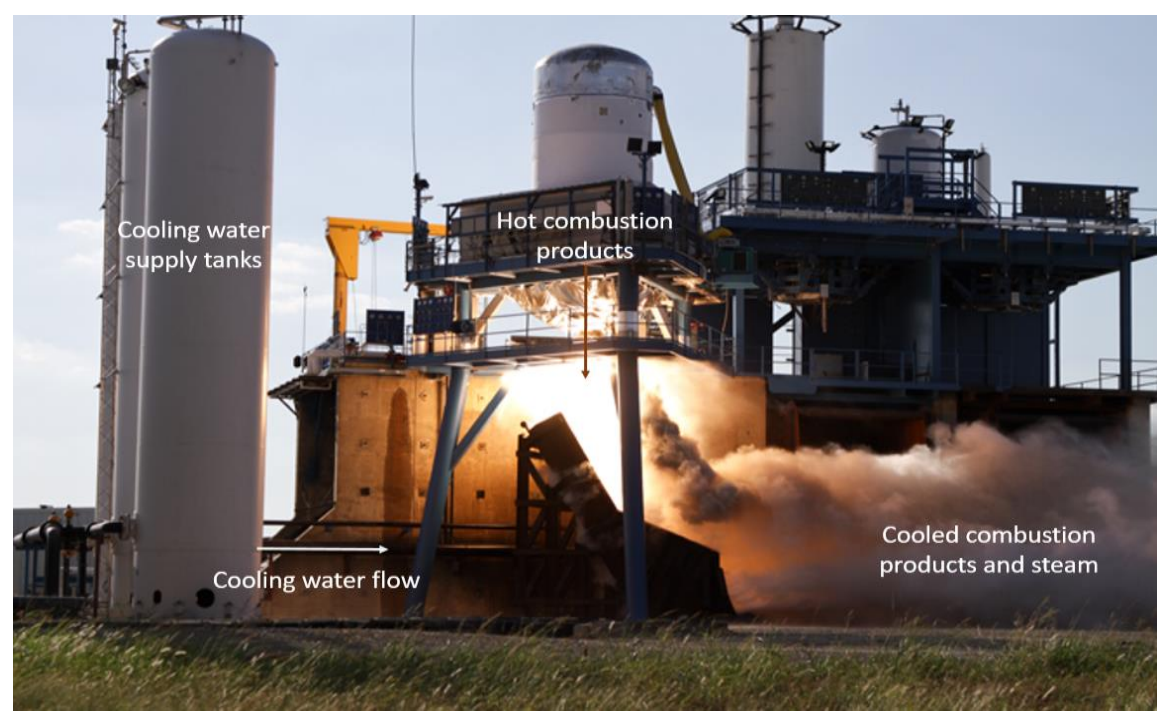

Figure 1. Water injection system in test stand[1] 
As many pump-fed cryogenic rocket engines are required to be fired vertically downward, this necessitates the use of a flame trench to manage the combustion products. As such there are a variety of solutions for injecting the cooling water including injection directly into an actively cooled flame trench as shown previously in Figure 1, by using spray ring upstream of an uncooled trench as shown in Figure 2, or by using a deflector plate with water spray holes as schematically shown in Figure 2. In either case, a variety of spray nozzles that can be varied in number, injection location, as well as spray distribution (coherent jet or pre-specified spray distribution) can be used. This introduction of liquid cooling has the effect of both cooling the combustion products by acting as an energy sink for liquid phase change and acting to disrupt the internal shockwave structure and strong turbulent eddies that are generated by the fast-moving supersonic gas flow, mitigating acoustic loading. In addition, there are water suppression systems which act to preserve test stand structure by providing a shielding water sheet to protect from radiative cooling, but they are not the subject of this work.
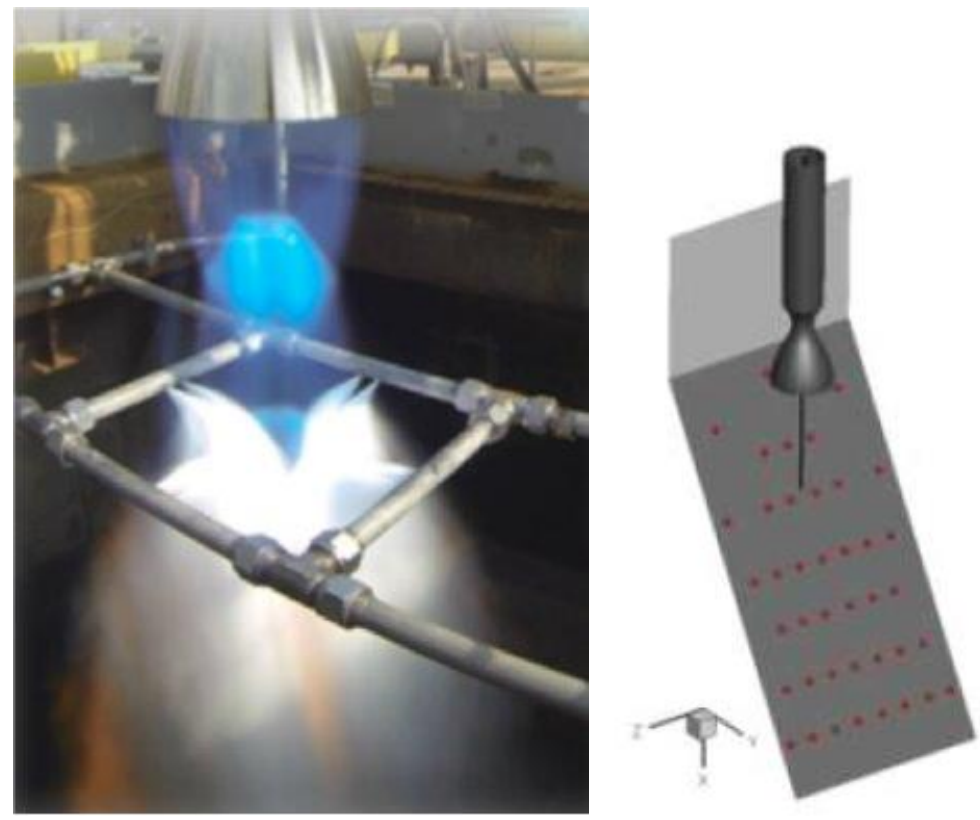

Figure 2: Left: Small scale test stand with spray ring[2], Right: Schematic showing deflector plate with water injection holes [3] 
As the proper design of such a system necessitates the analysis of a highly chaotic multiphase flow, computational fluid dynamics (CFD) methods have been leveraged by NASA engineers to be able to understand the complex behavior of these systems to design systems which are inherently safe and reliable. The CFD codes also permit optimization studies to evaluate cooling spray configurations that can provide optimal performance by utilizing the least amount of water for a rocket test. Typical water flow rates range from 30 to 50 times the rocket weight flow [5] and large-scale rocket tests require water flow rates in excess of 300,000 GPM. Thus, considerable saving in operations cost can be accomplished by an optimal cooling water spray design. Figure 3 below shows results from a study performed at NASA which explored the ability of a CFD code to properly predict the cooling behavior for a flat plate with perpendicular liquid injection. The study examined the temperatures experienced by the plate as well as the visual locations of maximum heating, and results showed good agreement with the simulated behavior.
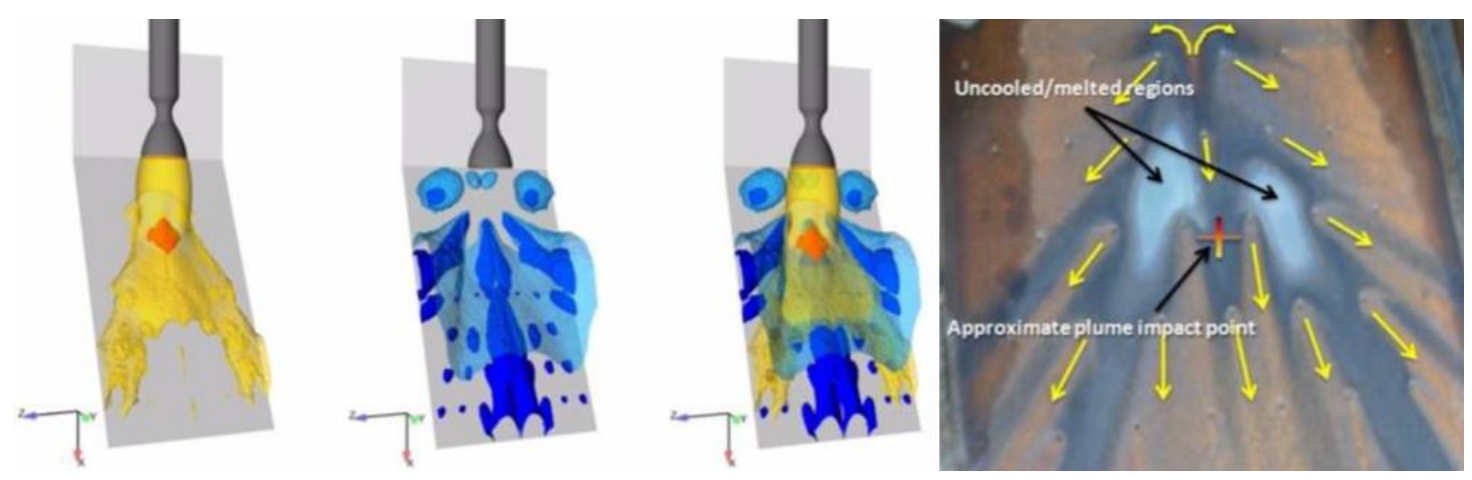

Figure 3. Left: CFD results for gas phase, liquid phase, combined phases, Right: Results for cooled plate[3]

Though promising results have been achieved for the global results of a computationally designed cooling system's ability to predict the cooling effects and locations, verifying the ability of the code to predict the breakup behavior of the liquid phase and understanding its interaction with the shock structure at the nozzle exit are rendered nearly impossible in the actual rocket tests at SSC. This is due to the highly chaotic, extremely harsh testing environment which makes it very 
difficult to conduct detailed qualitative and quantitative measurements of local flow properties that are needed to reliably verify the CFD code. Such flow properties include local temperature, flow velocity, and spray characteristics including droplet size and velocity which are required to be measured quantitatively. Qualitative measurements include overall spray structure, spray penetration, and changes to the shock structure induced by water injection. Figure 4 below demonstrates the harshness of the test environment as captured by CFD results where temperatures as high as $2600 \mathrm{~K}$ and flow velocities approaching Mach 3 and higher are developed at the rocket nozzle exit[4]. A further complication arising from the supersonic nature of the flow is that it necessitates the use of non-intrusive measurement techniques as any probe inserted in the flow will result in the disruption of the flow field by introducing shock structures upstream of the probe location.
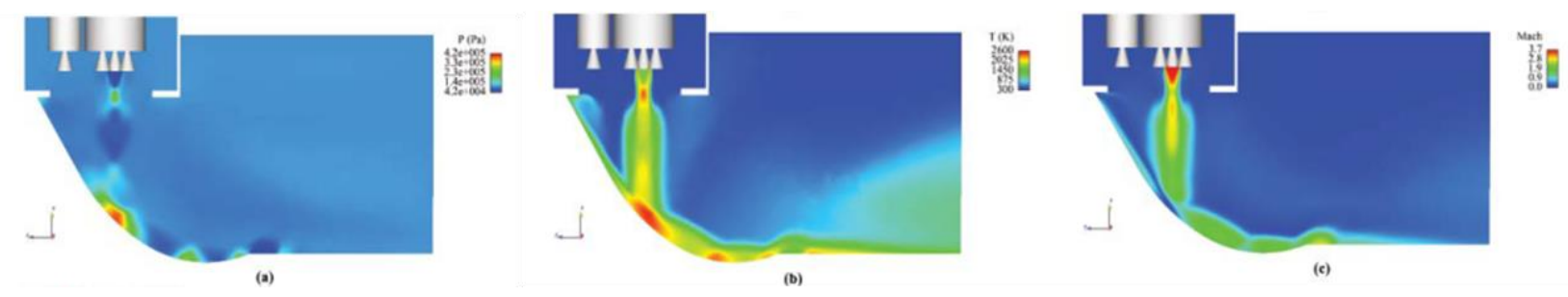

Figure 4. CFD results for Pressure (left), Temperature (middle), and Mach number (right)[4]

The need to validate the CFD code at SSC by obtaining qualitative and quantitative measurements using non-intrusive diagnostics in a laboratory test environment where all the relevant physical processes occurring in the rocket test can be reproduced in a laboratory test environment is the first key motivation for this work. The breakup of the liquid phase and its effects on the supersonic plume and associated shock structures are of key interest since they influence the ability of the CFD codes to predict spray droplet sizes and velocities which ultimately influence the heat transfer and acoustic suppression abilities of the water injection. 
While a number of simulation studies have been performed to study water injection into rocket exhaust including work in [3],[4],[12],[22], and [23], experimental studies are scarce and testament to the difficult test conditions. One study to note is by Liwu et al. [6] and later by Zhou et al [7] who investigated water injection into the exhaust plume of a solid rocket motor. A spray ring arrangement with 4 nozzles was utilized and measurements of spray structure and plume temperature were obtained using a high-speed camera and an infrared camera. While the temperature measurements were used to validate a CFD code, the results lacked any specific attempts to characterize the spray structure or the effect of the cooling water injection on the shock structure which are key motivations for the present work. Another set of relevant experimental studies is that performed on injection of cooling water jets into gas turbine exhaust for noise suppression. This includes studies by Norum[25], Krothapalli[24], Kandula, and others whose focus has primarily been the reduction of the overall sound pressure level for supersonic jet noise suppression. More detailed information of the spray breakup processes and its effects on acoustic suppression are available in these works including flow information obtained using Particle Image Velocimetry (PIV) and spray droplet and velocity information obtained using Phase Doppler Particle Anemometry (PDPA) [24].

Besides the two categories of relevant work identified in the discussion above (cooling water injection into rocket exhaust and jet engine exhaust), a third body of work that is related to the problem at hand is that of liquid jet injection into a supersonic crossflow. This is a very well researched subject area due to its numerous applications including fuel injection for supersonic combustion [19],[28]. However, it becomes interesting to examine the differences in behavior of liquid jet injection into a free jet, as is the case in the current work, and traditional jet in crossflow 
work. Figure 5 below shows schematically the differences in the two configurations, with jet in crossflow shown on the left and liquid jet injection into a free supersonic jet on the right.
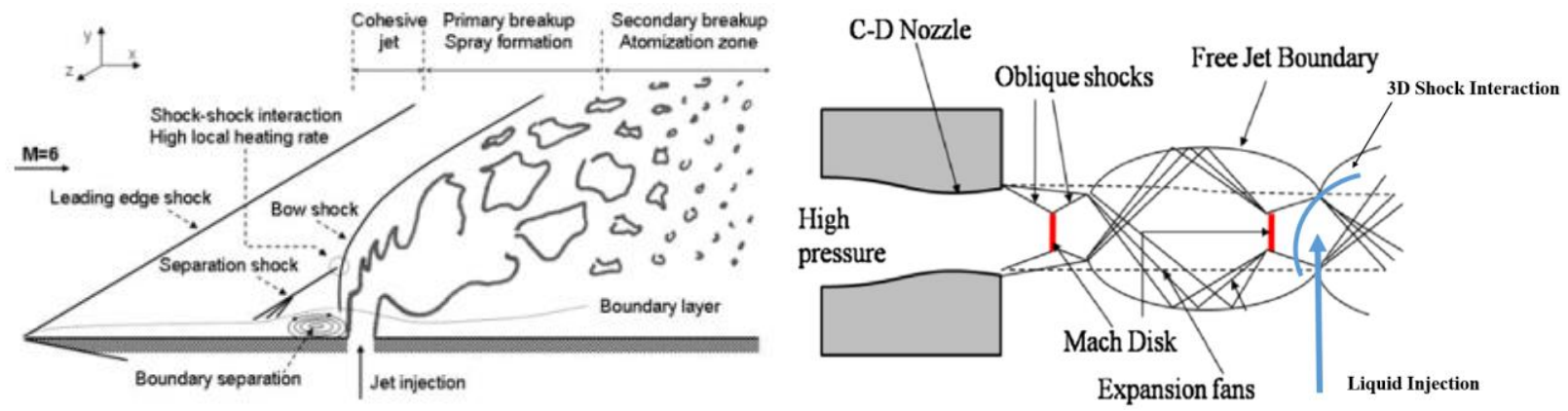

Figure 5. Left: Jet in crossflow [22], Right: Liquid penetration in a free jet [8]

Since the jet in crossflow is traditionally studied as it relates to fuel injection into supersonic combustion chambers, the upstream gas flow is generally uniform and contains no internal shockwave structure. For the injection configuration of interest in this work, the gas phase is expanded to a pressure less than ambient prior to injection into the ambient atmosphere, resulting in an overexpanded flow with an inherent internal shockwave structure. Additionally, the bounded nature of the traditional jet in crossflow allows the liquid flow to be influenced by the gas phase flow continuously while the free jet configuration is inherently 3 dimensional in nature, allowing the liquid phase to be displaced outside of the gas flow or even penetrate it completely. This lack of constraint in the case of the free jet as imposed by a solid boundary potentially introduces several changes in the flow structure from that in the traditional jet in crossflow and investigating these changes is the second key motivation for this work. An associated motivation is that of understanding the effect of water injection location in the shockwave structure of the overexpanded jet. Since the diameter of the water injection jet is small compared to the shock spacing, the interaction can change depending on the nature of the shock structure first seen by the injected jet. 
The third key motivation for this work arises from the liquid jet breakup process that takes place in the problem of interest. As will be discussed in detail in the following chapter, liquid jet breakup can be characterized into different regimes (capillary breakup, bag breakup, shear breakup, etc.) based on the jet to flow momentum ratio and the gas Weber number. As will be described in the following chapter, the breakup of water jets injected into rocket exhaust plumes lies in a very high Weber number regime (provide approximate value) which is outside of the regime diagram used to describe such flow configurations[28]. Obtaining a phenomenological model of the breakup process at this very high Weber number is the final motivation for this work.

The goals for this work are summarized below, with the main focus being to characterize the general penetration and breakup behavior of the liquid phase in a supersonic free jet crossflow using several non-intrusive diagnostic techniques.

1. Construct and characterize a system capable of delivering an overexpanded gas jet with variable water injection locations and conditions at a relatively steady-state condition to allow for proper measurements.

2. Examine qualitative differences in internal gas phase shock structure after liquid introduction compared with traditional jet in crossflow.

3. Obtain quantitative measurements of the spray structure by measuring water drop sizes and velocities.

4. Examine the regimes of initial liquid breakup and dominant physical forces creating the behavior at the very high Weber number (approx. value) range. The regimes of breakup should be consistent with what is present in some larger scale test environments. 
5. Examine the penetration distances for varied liquid injection conditions and compare with what is traditionally seen for jet in crossflow. The effect of varied liquid injection diameter to gas diameter ratio is to be explored as this is a key factor separating the two flow configurations.

\subsection{Thesis structure}

The following chapter provides a literature review examining the behavior of liquid breakup in traditional supersonic jet in cross flow and the available methods for examining shockwave behavior and liquid jet penetration as well as an introduction into the relevant theory. Here, an introduction to the behavior of liquid jet breakup and penetration as well as shockwave interaction with solid boundaries in 3D is presented.

Chapter 3 introduces the experimental methods used including Focusing Color Schlieren (FCS) for shockwave capturing, Phased Doppler Particle Anemometry (PDPA) for far field liquid breakup examination, and high-speed volume illuminated photography for capturing liquid penetration distance and initial qualitative breakup behavior. The operation of these methods is important as they allow for a nonintrusive approach to examining the behavior of breakup processes in supersonic flows.

Chapter 4 presents the results obtained for shockwave behavior, initial and far field break up and liquid penetration. These results are examined and compared to traditional liquid breakup in ducted crossflow.

Conclusions and recommendations as well as future work are presented last, including an introduction to the hybrid rocket that has been designed and is in production to be used as a next step in approaching the test conditions present in larger scale combusting flow environments. 


\section{Literature Review and Theory}

\subsection{Introduction to liquid jet in crossflow}

A jet injected at right angles into a crossflow is a configuration found in a wide range of engineering applications. This includes fuel injection applications in gas turbine and high-speed air-breathing engines, film cooling of gas turbine blades and other high stress applications, and thrust vector control in missiles [9]. A range of variations can accompany the traditional jet in crossflow configuration by having the injected jet or the crossflow being liquid, or gaseous, and reactive or non-reactive. With respect to fuel injection for propulsion applications, at high speeds the performance of the air breathing propulsion system often becomes limited by the ability of its combustor to fully utilize all fuel provided to generate meaningful thrust. The ability of a nonpremixed flame to sustain itself in a moving flow becomes more difficult as the flow speed approaches the flame speed of the combustion zone and mixing is limited by molecular and turbulent diffusion time scales. As such, in the design of such propulsion systems one must be very careful in the design of fuel injection systems, often in supersonic combusting environments. There are several strategies for keeping a flame steady in a supersonic combusting environment including flame holders which create a localized recirculation zone for lowering local flow velocity and allowing for significant combustion resonance time relative to the high mean flow velocity. There are several geometric designs for flame holders which balance drag with combustion stability but in each case one must be careful in examining jet penetration and breakup processes to ensure proper mixing downstream. The preceding discussion considers the injection of a reactive liquid fuel into a supersonic air crossflow. Despite the differences introduced by the phase of the fluid being injected and the presence or absence of chemical reaction, the physical structure of the interaction of a jet in a supersonic crossflow is generally the same and is analyzed next. This 
discussion provides context to the problem of interest in this research which is to analyze the flow phenomena for a free jet injected into a supersonic crossflow.

Figure 5 above shows schematically the generalized structure of both the gas and liquid phases in a typical jet in supersonic crossflow of constant Mach number. We can examine the gas and liquid phases separately for several important physical characteristics. As the liquid and gas phases are present at a very large density difference, the incoming supersonic flow sees the liquid phase as a solid boundary. This boundary effect generates a 3-dimensional bow shock present upstream of the liquid phase. While in general this shock is not stationary due to unsteady liquid breakup processes, this shock's location remains relatively constant in space depending on the upstream gas Mach number. Additionally, for this jet in supersonic flow there exists a secondary separation shockwave at the bounded liquid entry point due to supersonic boundary layer separation because of the adverse pressure gradient near the injection location. For the free jet in supersonic crossflow to be considered in the present work, this secondary separation shock is not expected due to the absence of a solid boundary.

The effectiveness of a fuel injection spray is a function of the penetration and mixing it creates as well as the ability of the fuel to be atomized. Determination of the spray penetration by tracking the liquid jet is an approach that has been extensively utilized to characterize the resulting jet penetration and mixing with the crossflow. Figure 6 shows the important features of a liquid jet injected into a uniform crossflow highlighting the details of the liquid jet breakup process. Two regions of the interaction can be distinguished: the trajectory of the jet up to the column breakup location and the subsequent generation and penetration of spray into the gas stream. Tracking the trajectories of both these regions and developing correlations for the jet penetration is the 
overarching problem which has been addressed in many previous publications using experimental and computational techniques.

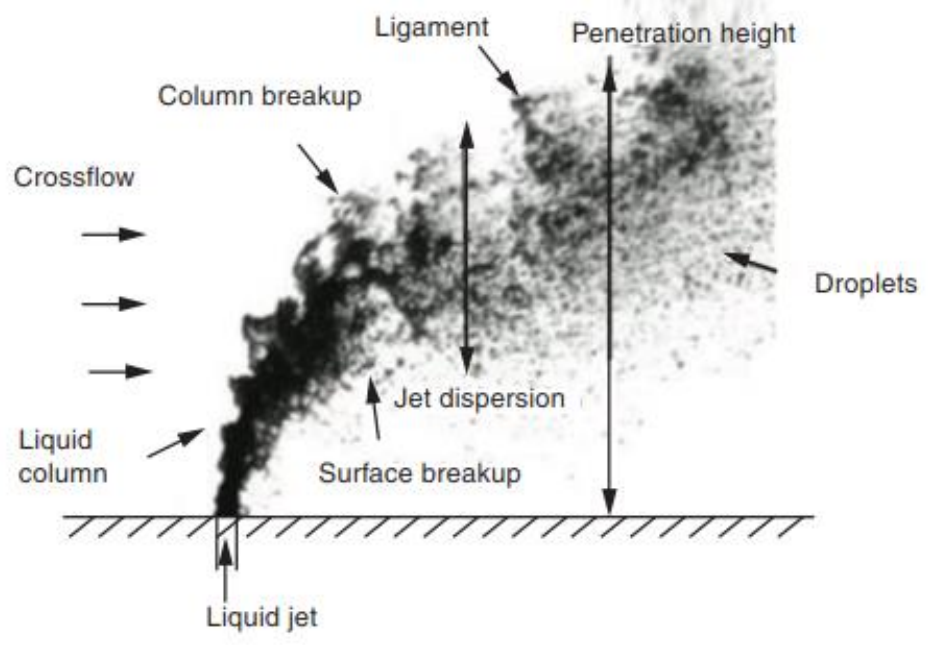

Figure 6. General distinguishing features of the liquid jet breakup in crossflow [17]

With regard to experiments, detailed surveys of correlations have been conducted by Ashgriz [10], Ragucci [11], Lin [12], Stenzler [13], Iyogun [14], McDonell [15], Marshall [16], and No [17]. The large number of empirical correlations can be classified based on functional form in the following ways:

1. Power-law: $\frac{y}{d}=A q^{\alpha}\left(\frac{x}{d}\right)^{b}$

2. Logarithmic: $\frac{y}{d}=A q^{\alpha}\left(1+\beta \frac{x}{d}\right)$

3. Exponential: $\frac{y}{d}=A q^{\alpha}\left[1-\exp \left(\beta \frac{x}{d}\right)\right]\left[1+B \exp \left(\gamma \frac{x}{d}\right)\right]\left[1+C \exp \left(\delta \frac{x}{d}\right)\right]$

Where $\mathrm{A}, \mathrm{B}, \mathrm{C}, \alpha, \beta, \gamma$, and $\delta$ are constants, $\mathrm{x}$ and $\mathrm{y}$ are horizontal and vertical distances, $\mathrm{d}$ is the liquid jet diameter, and $\mathrm{q}$ is the liquid to air momentum flux ratio. Other parameters including the Weber number (We), fluid viscosity ratio, pressure ratio, Reynolds number, and temperature ratio 
have also been incorporated into the correlations by various researchers. The general consensus appears to be that the jet penetration is chiefly a function of liquid to air momentum flux ratio, nozzle diameter and downstream distance along the gas flow from the injection point. The finite diameter of the air jet into which the liquid jet is injected as shown in Figure 5 (right) adds an additional dimensional parameter whose effect should be incorporated into the jet penetration correlation.

Among the large number of correlations, a correlation in the logarithmic form developed by Yates [19] was suggested in the initial stages of this work as being pertinent to the design of water spray injection systems at NASA SSC. Equation 2.1 below shows the correlation developed by Yates relating the penetration height $\bar{y}$ to the downstream distance $\bar{x}$, both of which are normalized by the effective orifice diameter $d_{e}=C_{d}^{0.5} d_{j}$ where $d_{j}$ is the geometric diameter is and $C_{d}$ is a discharge coefficient, and a liquid-gas dynamic pressure ratio $q=\frac{\rho_{l} v_{l}^{2}}{\rho_{g} v_{g}^{2}}$. There is a more complicated relation for the mean liquid penetration, which tracks the penetration of the center or densest portion of the spray, but as the edge penetration will be evaluated in this work we will only introduce the edge relation.

$$
\frac{\bar{y}_{B}}{d_{e}}=1.1 q^{0.5} \ln \left(1+10 \frac{\bar{x}}{d_{e}}\right)
$$

This relation appears to hold well for flows in traditional jet in crossflow as shown in Figure 5 where the liquid flow is constrained within the gas flow throughout its travel, as such equation 2.1 displays asymptotic behavior approaching a steady state penetration for large $\frac{\bar{x}}{d_{e}}$. Whether or not, a similar behavior applies for a free jet injected into a supersonic crossflow is one of the motivations for the present work. 
A variety of experimental techniques have been used by previous researchers to generate jet penetration correlations including photography, cinematography, shadowgraphy, holography, Mie-scattering, and Phase Doppler Particle Anemometry (PDPA). Digital holography is a more advanced technique which allows for the reconstruction of a 3D field of objects and examined in distinct 2D sections using slicing techniques. This approach can be used to obtain high fidelity experimental data not previously available. Figure 7 below shows results obtained via this technique which allow for particle detection and sizing in 3D for a given jet in crossflow configuration[21]. Where the previous techniques examine more qualitatively the characteristics of liquid phase penetration and primary breakup mode, this technique allows a more quantitative picture of droplet distribution to be built.
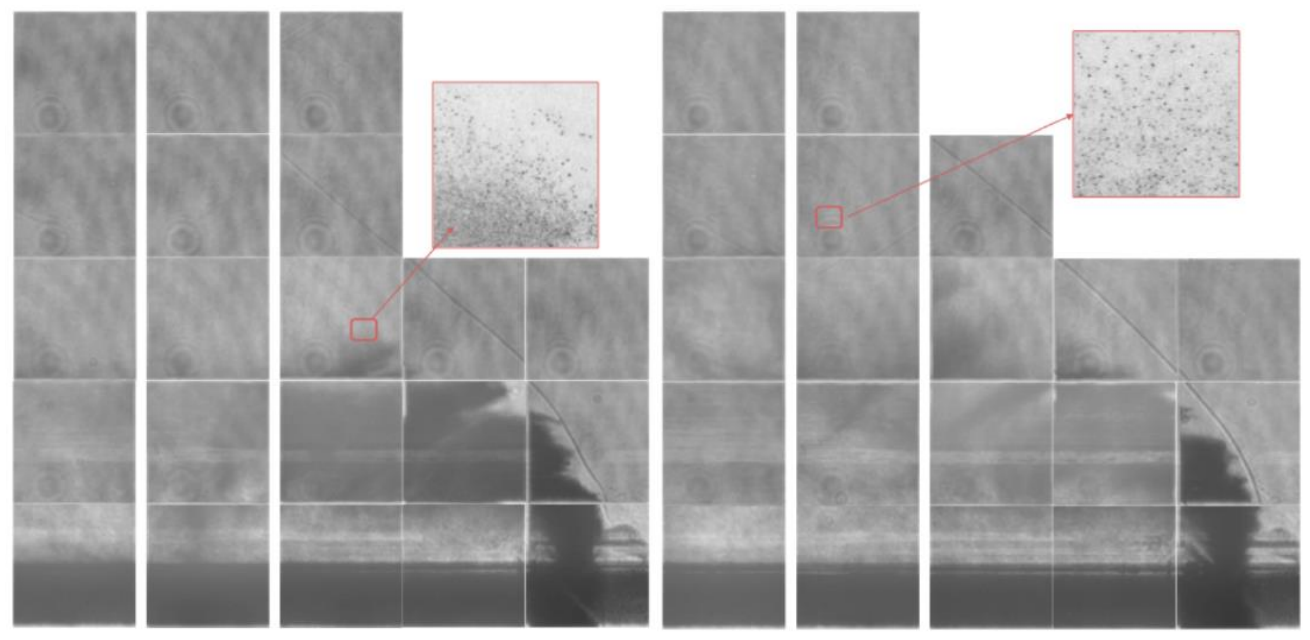

Figure 7. Left: Upstream particle detection using holography, Right: Downstream particle detection using holography[21]

More recently, detailed numerical simulations have employed computational fluid dynamics (CFD) techniques to gain insight into jet penetration and breakup [3],[4],[12],[22],[23]. Figure 8 below shows numerical results from one such work obtained by Liu et. al. for a two-fluid case with water injected into a supersonic air crossflow. Good agreement is shown between the model predictions for jet penetration and several experimental correlations for a momentum ratio 
of $\mathrm{q}=6$. In addition, the simulation results capture the major flow structures including the bow shock and separation shock and the separated recirculation region near the surface boundary, all of which are observed in experimental results.
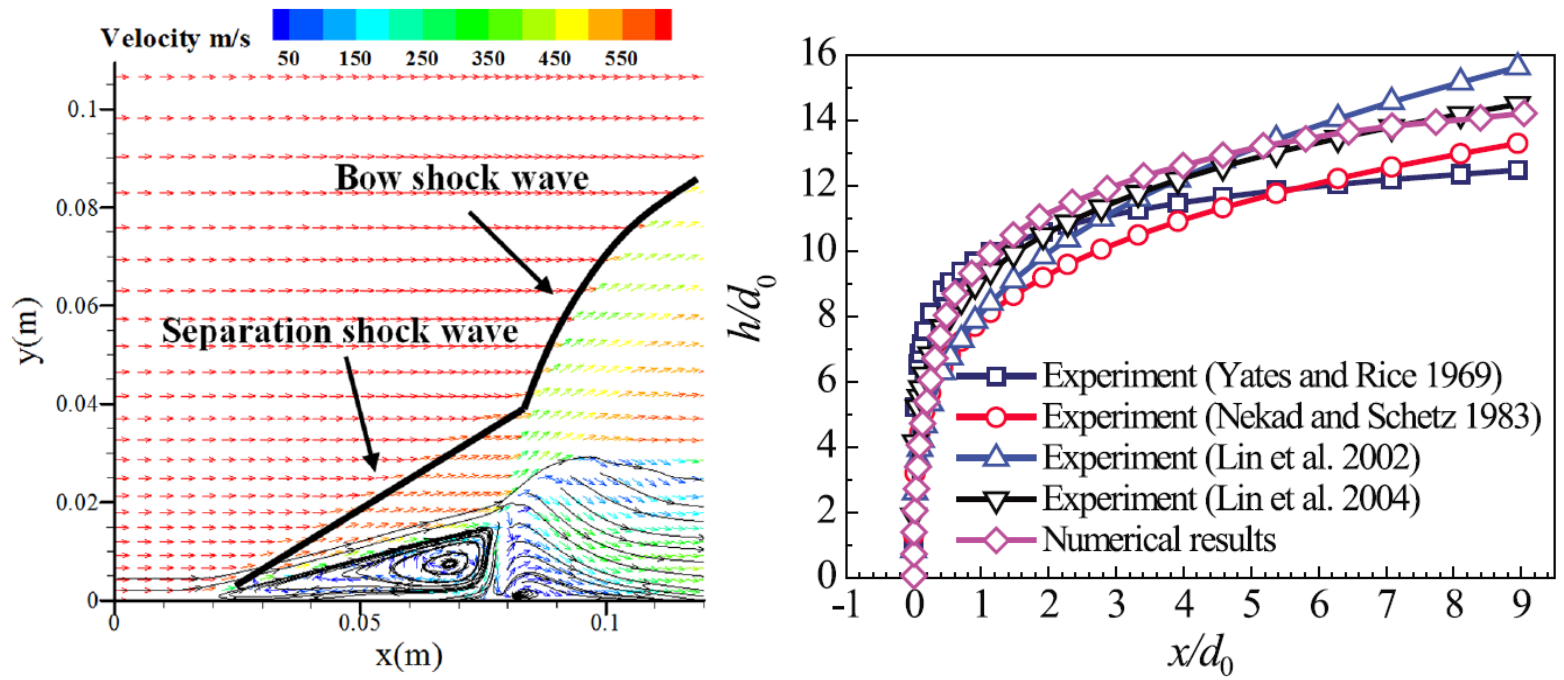

Figure 8. Left: Velocity contour plot showing shockwave locations, Right: Numerical results compared with prior experimental results for various momentum ratios[22]

CFD simulations have the additional advantage of being able to examine in great detail, the behavior of the primary breakup processes for varied gas phase Mach number and momentum ratio as shown by [23]. The objective of this work was to examine the regime of breakup and the global effects of the solid boundary created by the introduction of the liquid phase. Figure 9 below shows results obtained by this work for varied momentum ratios of $q=3$ and $q=6$ with the morphology of the liquid phase and pressure iso-contours plotted. It is important here to note the changes in the liquid morphology for increased liquid dynamic pressure, the physics of these changes will be discussed further in the following sections. 


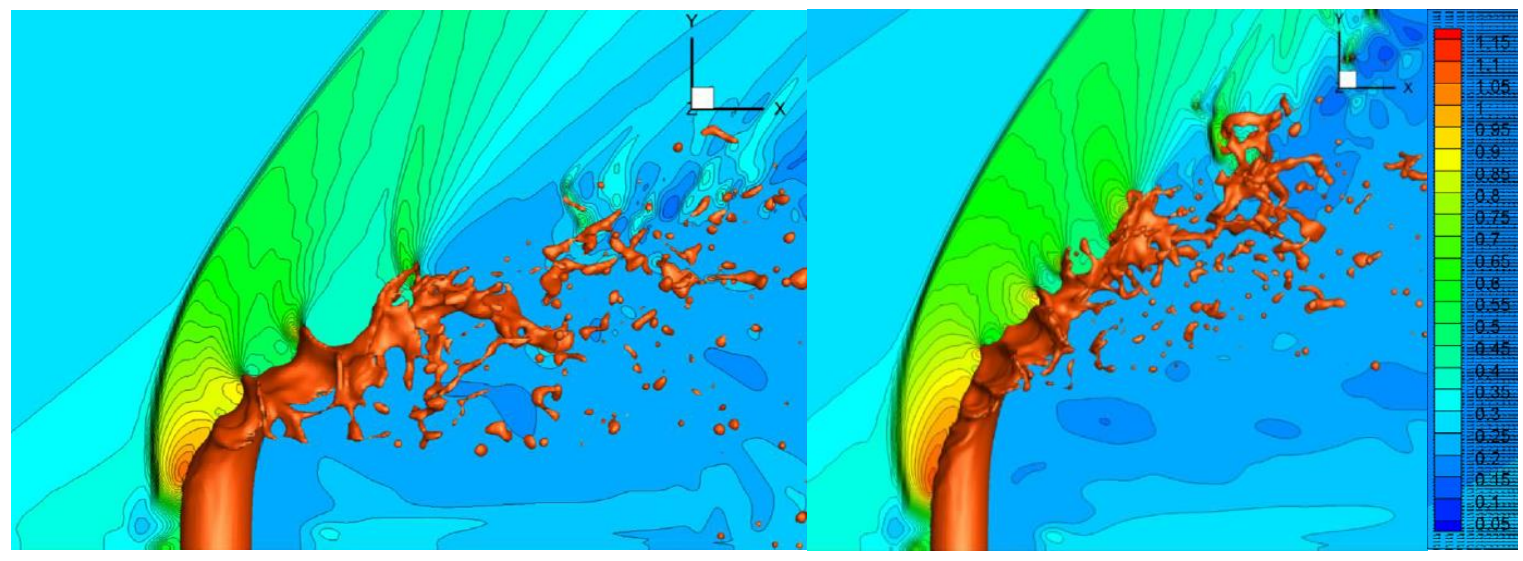

Figure 9. Left: Morphology for q=3, Right: Morphology for $q=6$ [23]

All previous work discussed has been applied to traditional jet in crossflow, a configuration where the liquid phase is injected into a gas flow where the liquid's residence time is long compared to free jet injection, allowing for predictable behavior of the liquid phase following the gas phase. However, the current work examines behavior for injection to a free supersonic gas jet where the presence of an internal shockwave structure can alter the energy content of the gas phase.

There is a considerable amount of past work relating to the injection of liquid water into high speed gas flows, both hot and cold, for the purpose of suppressing noise. The variation in noise reduction has been heavily explored for the best location and orientation relative to the gas flow exit location to minimize the overall sound pressure level (OASPL) generated. While there is a large amount of previous work related to the noise reduction problem, much of it is focused on the effect of jet injection on sound suppression. The liquid phase breakup and jet penetration effects have not been explored to the same degree in these investigations. Figure 10 below shows an experimental setup employed at NASA Langley Research Center to examine the effects of liquid injection into supersonic exhaust using microjets and the resulting OASPL[25]. 


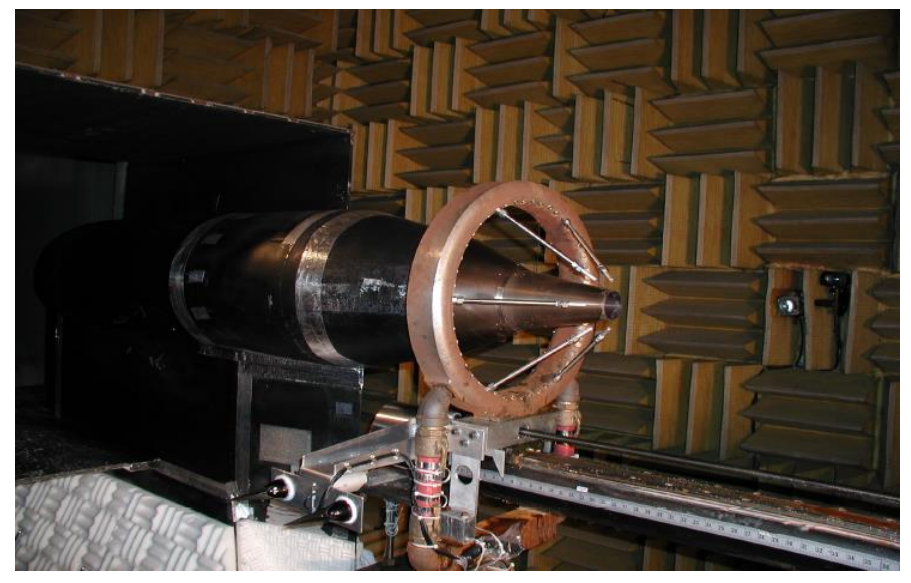

Figure 10. Microjet injection into jet engine exhaust [25]

The work related to the setup shown in Figure 10 as well as other work involving microjets explore noise reduction as a function of the momentum ratio [26][27]. However, the studies on traditional jet in crossflow and those that focus on noise reduction incorporate differing ways of defining the momentum ratio. For the studies relating to noise reduction as well as the case of interest in this work, the effect of the relative size of the two jets is important and needs to be considered. With regard to work involving traditional jet injection into crossflow, equation 2.2 below shows a common definition, which is utilized in the above equation 2.1 developed by Yates. An important note here is that for the jet in crossflow configuration, the gas phase momentum is easily calculated as the flow is generally uniform in cross-section and the crossflow is sufficiently larger than the injected jet that it acts as an ambient environment into which the jet is injected.

$$
q=\frac{\rho_{\text {liquid }} v_{\text {liquid }}^{2}}{\rho_{\text {gas }} v_{\text {gas }}^{2}}
$$

When examining a free supersonic gas jet with interior shock structure one must consider the changes in total pressure of the flow due to the presence of shockwave irreversibilities in addition to the finite sizes of the two jets. As such, in lieu of gas momentum, several studies have used the gas dynamic pressure once the jet is isentropically expanded to ambient pressure with the 
motivation of using this as a measure of overall energy. Still other studies have simply used gas phase total pressure.

With the current work seeking to examine the behavior of liquid injection into a free jet, it becomes apparent that one must consider injection diameter as a variable in the interaction behavior. Previous studies also focused on examining reduced OASPL for free supersonic jets of various temperature have seen good agreement with the definition of momentum ratio as shown in equation 2.3 below[27]. Here, the pressure $\mathrm{P}$ represents total pressure while $\mathrm{d}$ is the geometric jet diameter.

$$
q=\frac{P_{\text {liquid }} d_{\text {liquid }}}{P_{\text {gas }} d_{\text {gas }}}
$$

Referring to equation 2.3 gas phase energy is represented by total pressure, and the fully expanded Mach number $M_{j}$ (expanded to ambient) can easily be recovered for a given ambient pressure $P_{a}$ for a known total pressure and ratio of specific heats $k=\frac{c_{p}}{c_{v}}$ as is shown in equation 2.4 below. As the effect of nozzle diameter change is of interest, the definition presented in (2.3) will be employed in this work, with $\mathrm{k}=1.4$ being used as the gas of interest will be air.

$$
M_{j}=\left(\left(\left(\frac{P_{o}}{P_{a}}\right)^{\frac{k-1}{k}}-1\right) \frac{2}{k-1}\right)^{\frac{1}{2}}
$$

\subsection{Shockwave behavior in free supersonic jets}

A key difference between traditional jet in crossflow and the free supersonic jet configuration is the presence of an internal shock structure. As the gas jet is accelerated through a converging-diverging (C-D) nozzle, enthalpy in the flow is traded for kinetic energy, and the 
pressure of the resulting downstream flow decreases. This decrease in pressure with increasing Mach number can result in differing internal shockwave structure in the free jet depending on the ambient pressure $P_{a}$ compared with the static pressure at the nozzle exit $P_{e}$. In a free jet where $\frac{P_{e}}{P_{a}}>$ 1 the jet is said to be underexpanded, meaning that the gas flow has not expanded enough to reach ambient pressure. On the other hand, when $\frac{P_{e}}{P_{a}}<1$ the jet is overexpanded, meaning it has been expanded enough to reach a pressure less than ambient. Figure 11 below shows schematically the general shockwave structure present in an overexpanded and underexpanded free jet. The largest difference is the presence of an initial oblique shock in the overexpanded case as it interacts with the higher pressure ambient gas and an initial expansion region following the nozzle exit in the underexpanded case as it expands isentropically to ambient pressure. In each case, an oblique shock train follows downstream with each set of intersecting waves commonly referred to as "Mach diamonds". 

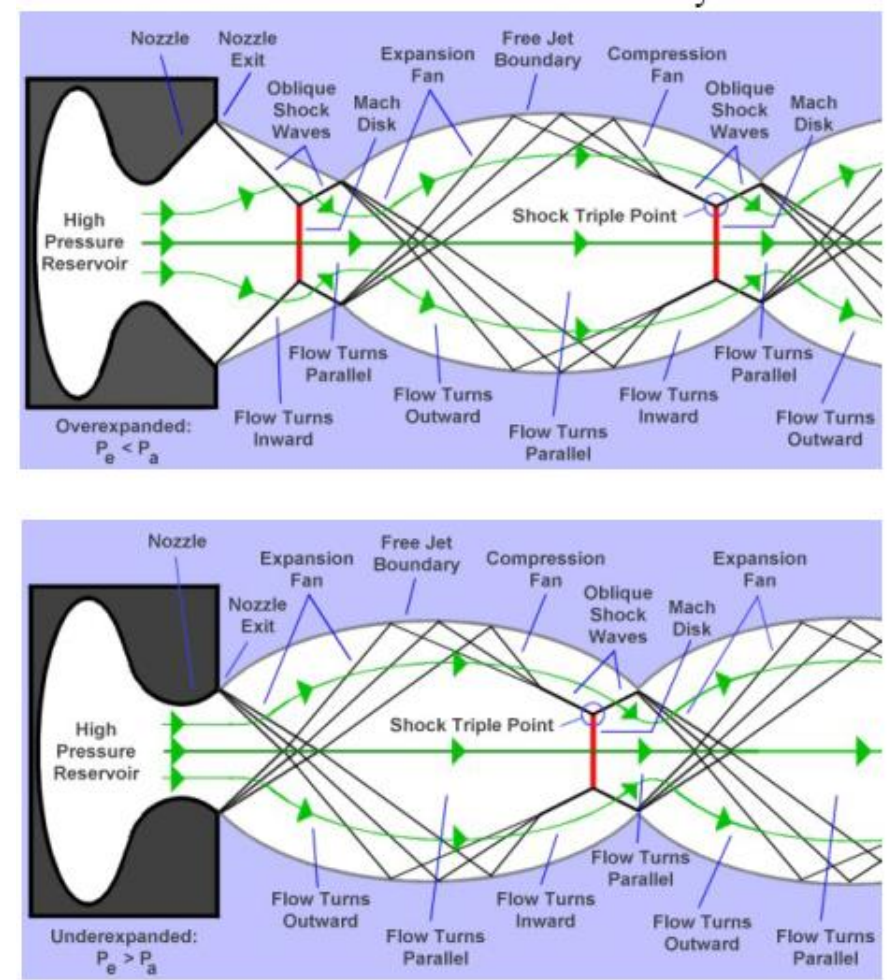

Figure 11. Top: Overexpanded gas jet shock structure, Bottom: Underexpanded jet shock structure [18]

For both the cases shown above, the shockwaves will propagate in a largely inviscid potential core flow until the viscous dissipation present in the surrounding mixing region decelerates the flow to subsonic velocity. Equation 2.5 shown below describes oblique shock reflection angle $\beta$ as a function of upstream Mach number $M_{1}$, solid surface angle relative to flow direction $\theta$, and gas specific heat ratio $k=\frac{c_{p}}{c_{v}}$. This equation is appropriate up to certain boundary angle $\theta$ such that there is no solution for $\beta$. For such cases, physically this indicates that the shock wave has separated from the boundary to form a bow shock. For a liquid jet injected into a supersonic crossflow, the bow shock is commonly observed as seen in Figure 5.

$$
\tan \theta=2 \cot (\beta) \frac{\left(M_{1}^{2} \sin ^{2} \beta\right)-1}{M_{1}^{2}(k+\cos 2 \beta)+2}
$$


For low shockwave angles, the changes in the flow's total pressure can be minimal as the change is proportional to the flow Mach number normal to the oblique shockwave $M=M_{1} \sin \beta$, which can be small as $\frac{P_{t 1}}{P_{t 0}} \propto M^{2}$ with $P_{t 1}$ and $P_{t 0}$ representing the total pressure downstream and upstream respectively. This relationship is shown in equation 2.6 below.

$$
\frac{P_{t 1}}{P_{t 0}}=\left[\frac{(k+1) M^{2}}{(k-1) M^{2}+2}\right]^{\frac{k}{k-1}}\left[\frac{(k+1)}{2 k M^{2}-(k-1)}\right]
$$

As a result, large angular changes in flow direction can have a large impact on the gas flow momentum. This change can be manifested as a decrease in OASPL as further downstream shockwaves and mixing is inhibited or as an increased penetration distance in free gas jets.

\subsection{Liquid jet primary breakup processes}

There are many practical applications such as fuel injection and fire suppression where the introduction of a spray, with known particle size and distribution, plays a key role in the ensuing process. To this end, the parameters governing particle size and distribution are optimized to achieve the desired spray structure. However, for the configuration studied in this work, the liquid phase is injected first as a coherent jet and is subsequently broken up following its interaction with the gas phase. Hence it is important to discuss the physics of the jet breakup process.

First, we introduce several non-dimensional numbers which will allow us to discuss the primary breakup regimes for crossflows. In addition, the secondary breakup process will be introduced but not expanded upon as it is not heavily explored in this work. Primary breakup refers to the breakup of the initial jet structure through column breakup induced by instability waves or shear breakup induced by surface stripping. Secondary breakup refers to the subsequent 
fragmentation of the drops formed by primary breakup [28]. Figure 12 shows the key features of the primary breakup process to be analyzed in this work.

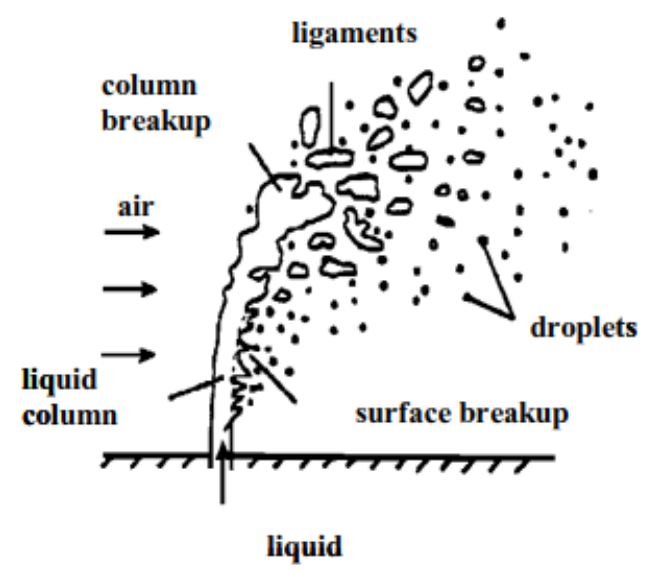

Figure 12. Breakup process of the jet illustrating column and shear breakup [28]

Equation 2.7 below shows the expression for the gas Weber number, which represents the ratio of inertial forces to surface tension forces at a two-phase fluid interface. Here, the density $\rho$ and velocity $v$ are taken for the gas phase and the surface tension $\sigma$ for the liquid phase, $l$ represents a characteristic length for the flow taken as the diameter of the coherent liquid jet prior to injection for the cases to be considered.

$$
W e_{G}=\frac{\rho v^{2} l}{\sigma}
$$

Along with the gas Weber number to quantify the relative importance of gas momentum to liquid internal forces the regime of initial liquid breakup can be characterized based on the momentum ratio $q$ discussed previously. Figure 13 below shows a regime map obtained by plotting the momentum ratio as a function of the gas Weber number and delineating the regions exhibiting a specific breakup mode[28]. For supersonic flows where the gas velocity far exceeds the liquid injection velocity, we tend to see very low liquid-gas momentum flux ratios $q$ and very high gas Weber numbers $W e_{G}$. This would imply that for the configuration investigated in this work, the 
initial liquid breakup is largely shear dominated as the energy content in each phase is vastly different. This puts the location of the jet interaction investigated in this work towards the lower right corner of the regime diagram.

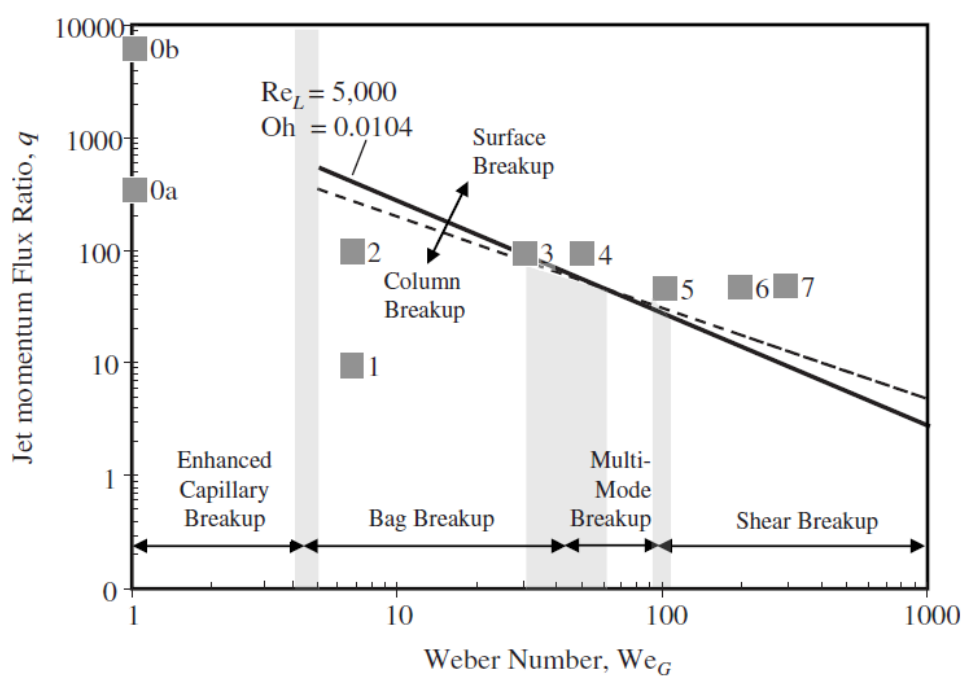

Figure 13. Initial breakup regime map for two fluid interfaces[28]

Physically, a high gas Weber number low momentum ratio shear breakup process would be characterized by a gas phase dominated flow where "packets" of liquid are sheared from the incident coherent jet and individually atomized in the gas flow. This process is fundamentally different from column breakup for low momentum ratio flows with a lower gas Weber number as the characteristic "columns" are unable to form downstream of the injection point. This column or ligament breakup process is common in lower speed or subsonic liquid breakup processes but not in the current work as the gas Weber number is so large. At increased momentum ratio surface stripping effects are present, as the larger liquid momentum increases the ability of the liquid jet to propagate in the gas flow and liquid is first sheared from the coherent surface prior to atomization downstream rather than being broken into longitudinal segments. Figure 14 below shows the liquid morphology for the column and surface breakup regimes $q=3$ and $q=18$ in the left 
and right image respectively for a Weber number of $\sim 800$, resulting in dramatically different penetration and breakup behavior. Shear breakup behavior will be explored and shown later in this work as it is present in the regime of the current work.

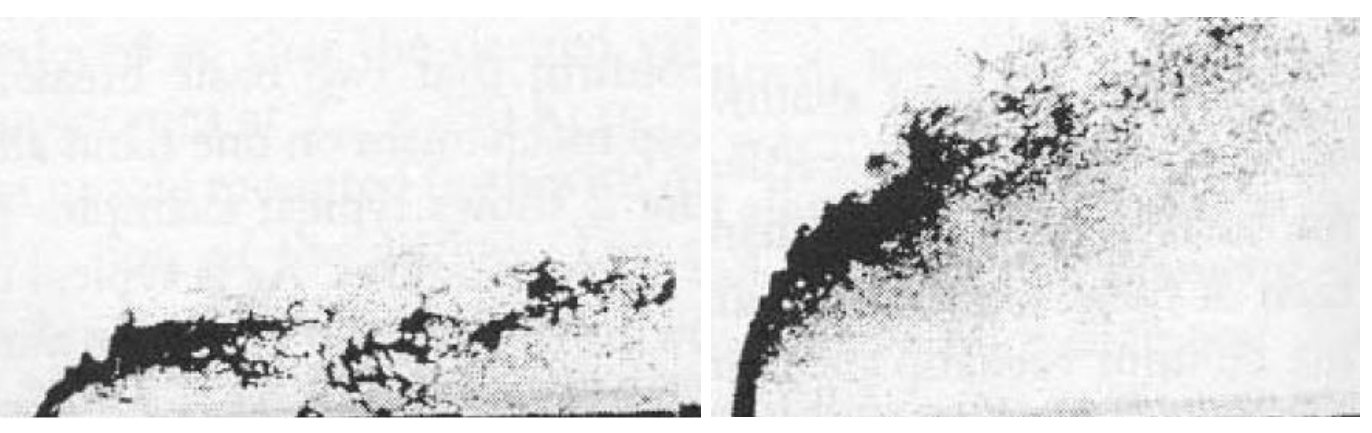

Figure 14. Left: Column breakup, Right: Surface breakup[28]

Following the primary breakup phase as seen in Figure 14 and shown schematically in Figure 5, a secondary breakup zone forms where the initial breakup formed by disturbances to the coherent jet structure are further atomized. The effectiveness of this atomization process in generating a well dispersed spray plays a key role in applications such as fuel injection as well as in water spray cooling for rocket exhaust plumes which is of interest in this work. Characterizing the spray structure by measuring particle size and velocity in this zone is of great importance to the success of validation cases provided for the NASA code.

Another important parameter to examine is the Stokes number, St. This dimensionless parameter is used to quantify how well particles suspended in a fluid flow follow the global motion. Equation 2.7 below displays the definition for the stokes number where $u_{o}$ represents the mean flow velocity, $t_{o}$ is the characteristic relaxation time for the particle, a measure of its time to change velocity due to drag, and $l_{o}$ is a particle characteristic length, typically taken to be the particle diameter.

$$
S t k=\frac{t_{o} u_{o}}{l_{o}}
$$


A particle with a low Stokes number will follow the global fluid flow well while for a particle with a large stokes number, its motion is dominated by inertia and it will not follow the mean flow as well. This information can allow us to determine spatial locations in our separated flow area of interest where the flow may or may not be influenced by the gas jet, as the free jet will experience flow turning at larger momentum ratios. Additionally, checking this value serves as a sanity check for experimental results where droplet velocities are measured since the gas flow velocity is approximately known and a rough estimate of droplet velocity can be obtained by knowing the Stokes number.

\subsection{Hybrid rocket design considerations}

While the major focus of this thesis is on the interaction of a supersonic air jet with a free jet of water motivated by the cooling water injection process used in rocket testing, it primarily considers a cold supersonic air jet. Ongoing work is also considering the use of a scaled hybrid rocket system to generate a combusting free jet to better replicate conditions present on the test stands at NASA SSC. As such it is important to briefly introduce some concepts relating to the operation of the hybrid rocket engine. Much like with the non-combusting cold flow system used in the current work, the hybrid rocket engine is designed to generate a high speed overexpanded gas jet. In contrast to traditional liquid rocket engines like the RS-25 used to power the space shuttle and future space launch system (SLS) and solid motors like those used as boosters to power the space shuttle, a hybrid rocket engine stores oxidizer and fuel, the components necessary to sustain combustion, in two different phases. While larger scale hybrid engines which are focused on delivering high levels of thrust will generally use a liquid phase oxidizer, a gas phase oxidizer is being used in the work to be conducted at LSU as it is safer to handle and is easier to control on

a small scale to produce steady combustion. Figure 15 below shows schematically the general 
layout of a hybrid rocket engine system. The oxidizer is stored separately with flow into the combustion chamber, where a solid fuel is held, controlled via a single valve; the combustion process is started using an igniter or similar system. The high pressure, high temperature gases generated in the combustion chamber are then expanded through a nozzle section to accelerate the flow, providing the kinetic energy to the gas flow necessary to generate useful thrust.

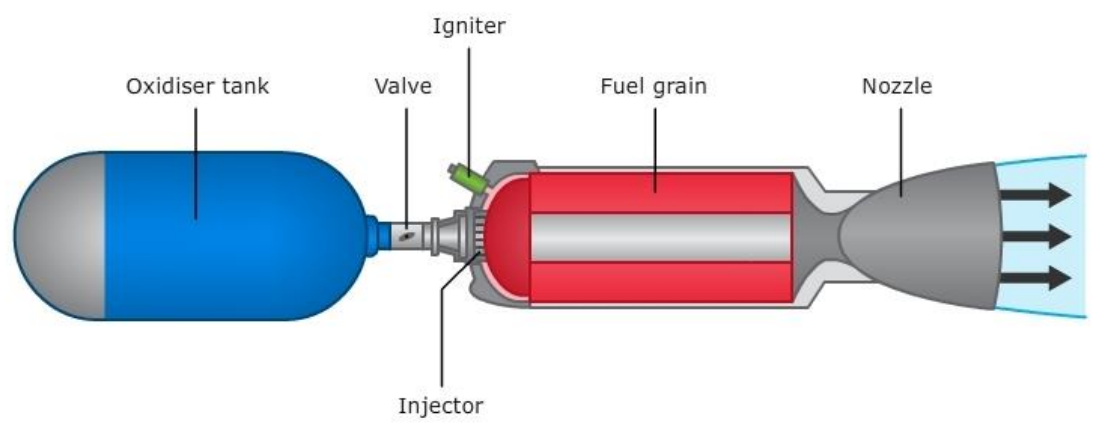

Figure 15. Hybrid rocket engine schematic [29]

In a similar manner to the operation of the cold flow nozzle used in the current work, the job of the systems upstream of the nozzle is to generate useful total energy in the form of total temperature and pressure which can be accelerated through the nozzle. Equation 2.8 below shows how total pressure in the combustion chamber $P_{o}$ depends on mass flow rate through the choked nozzle throat (location where Mach number is 1 ) $\dot{m}$, the throat area $A^{*}$, ratio of specific heats $\mathrm{k}$, specific gas constant $\mathrm{R}$, and total chamber temperature $T_{o}$.

$$
P_{o}=\frac{\dot{m}}{A^{*}}\left[\sqrt{\frac{k}{R T_{o}}}\left(\frac{2}{k+1}\right)^{\frac{k+1}{2(k-1)}}\right]^{-1}
$$

It can be seen here that for maximizing chamber pressure one should seek to maximize mass flow rate, specific gas constant and total temperature, while minimizing $\mathrm{k}$, and the nozzle throat area. However, maximizing the chamber pressure is only important when seeking maximum 
thrust. The relevant focus of this work is to generate specified values of static pressure and temperature, as well as Mach number at the nozzle exit consistent with conditions observed at the test stands at NASA SSC. For the current work, it is important that the gases exiting the nozzle are expanded to a static pressure less than ambient to provide an overexpanded jet. Equation 2.9 below shows the isentropic relation which can be used to calculate an exit Mach number $M_{e}$ given a known value of $P_{o}$ and chosen exit pressure $P_{e}$. This relation applies to a gas flow which is assumed to be calorically perfect, isentropic, and one-dimensional (computed values are an average at a given location in the nozzle).

$$
\frac{P_{o}}{P_{e}}=\left(1+\frac{k-1}{2} M_{e}^{2}\right)^{\frac{k}{k-1}}
$$

Similarly, one can determine the necessary expansion ratio in the supersonic section of the nozzle required to generate a flow for a given Mach number, allowing for proper design of a nozzle to achieve design requirements. Equation 2.10 below shows this expansion ratio as a function of exit Mach number and gas ratio of specific heats.

$$
\frac{A_{e}}{A^{*}}=\frac{1}{M_{e}}\left(\frac{2}{k+1}\left(1+\frac{k-1}{2} M_{e}^{2}\right)\right)^{\frac{k+1}{2(k-1)}}
$$

Examining equation 2.8 again shows that we must next consider the gas total temperature, that being the gas temperature in the combustion chamber where flow velocity is relatively low. This property is purely dependent on the properties of combustion for the reactants chosen to be used in the engine. As residence times in most engines are long compared with the time scale of combustion an equilibrium solver is generally used to compute the combustion temperature. Solving for the equilibrium composition and temperature is advantageous most laboratory scale 
hybrid rockets burn fuels consisting of long chain hydrocarbons, which can generate very complex combustion chemistry. The equilibrium solver used in this work is the Chemical Equilibrium with Applications (CEA) software freely available through NASA's Glenn research center [30]. The results for this chemical equilibrium computation are dependent on the o/f ratio, or the ratio of mass of oxidizer to mass of fuel used in combustion. Choosing this property correctly is important as it affects many aspects of the combustion process including overall efficiency and combustion temperature. Figure 16 below shows a sample plot generated for one such property, specific impulse, which is a measure of engine efficiency. Results are presented for the same fuel planned for use in future work, hydroxyl-terminated polybutadiene (HTPB).

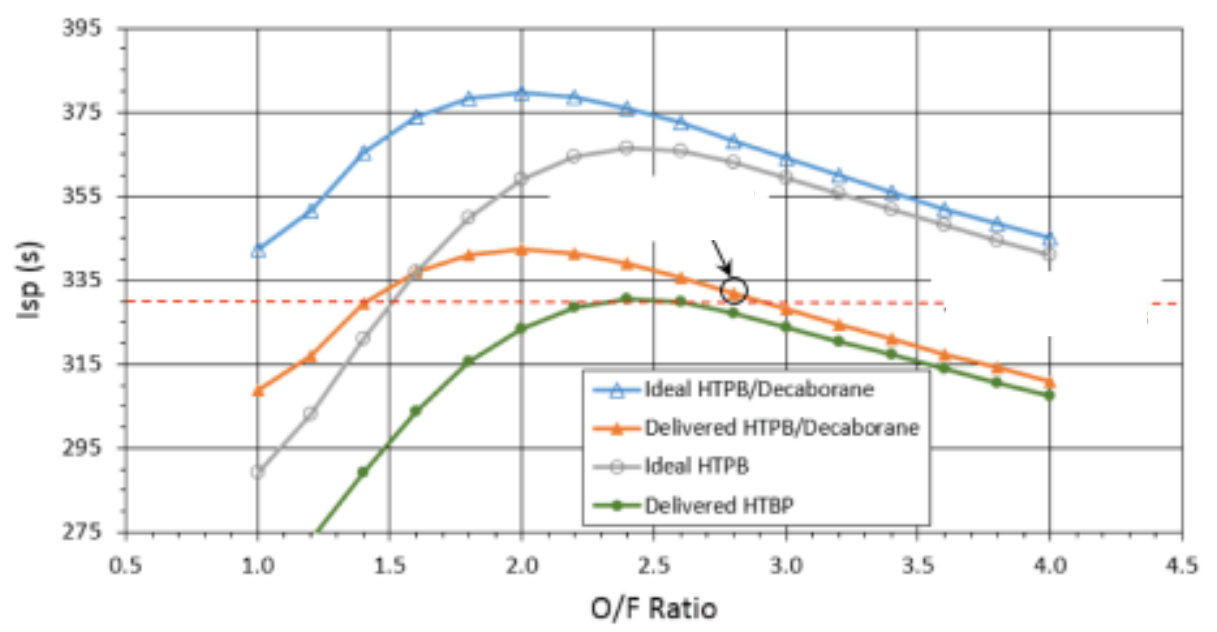

Figure 16. Sample specific impulse as a function of olf for HTPB fuel [31]

The location of maximum specific impulse in the below plot corresponds to the location of stoichiometric combustion, or the o/f where the products of combustion are only carbon dioxide and water. Though this o/f will produce the most chamber pressure possible it also will produce the largest combustion temperature. Generally, operation at the stoichiometric o/f ratio is avoided. it is also difficult to achieve a large enough fuel flowrate to operate at this point, the means of 
determining fuel flowrate is to be determined next, with low fuel burn rates for HTPB shown in Figure 17 being used to choose this as a fuel for a non-thrust-limited stable combustion system.

With the remaining parameters governed by gas properties, the effectiveness of a propellant's ability to convert chemical energy to heat, the mass flow rate through the system must now be determined. This total mass flow is comprised simply of the combined flow rate of the gaseous oxidizer and solid fuel. While the flow rate of the oxidizer is determined easily for a choked upstream condition (which is generally desired for combustion stability and safety) the mass flow rate of the solid phase fuel is more difficult to determine. Due to the complex nonpremixed combustion process occurring in the combustion chamber the flow rate of fuel for a given oxidizer-fuel combination and oxidizer flow rate is generally determined experimentally. Figure 17 below shows such an experimentally determined relationship for an $\mathrm{O}_{2}$ hybrid system for various solid fuels including HTPB and paraffin wax. As can be seen, there is an increase in fuel regression rate for all fuels as the mass flux of oxidizer $\frac{m_{o x}}{A_{\text {chamber }}}$ through the combustion chamber increases, this is the important data of interest when setting design points for oxidizer flowrate, fuel type, and combustion chamber size. At oxidizer mass flux values above and below the range showed here the combustion process will generally become unstable and should be avoided. 


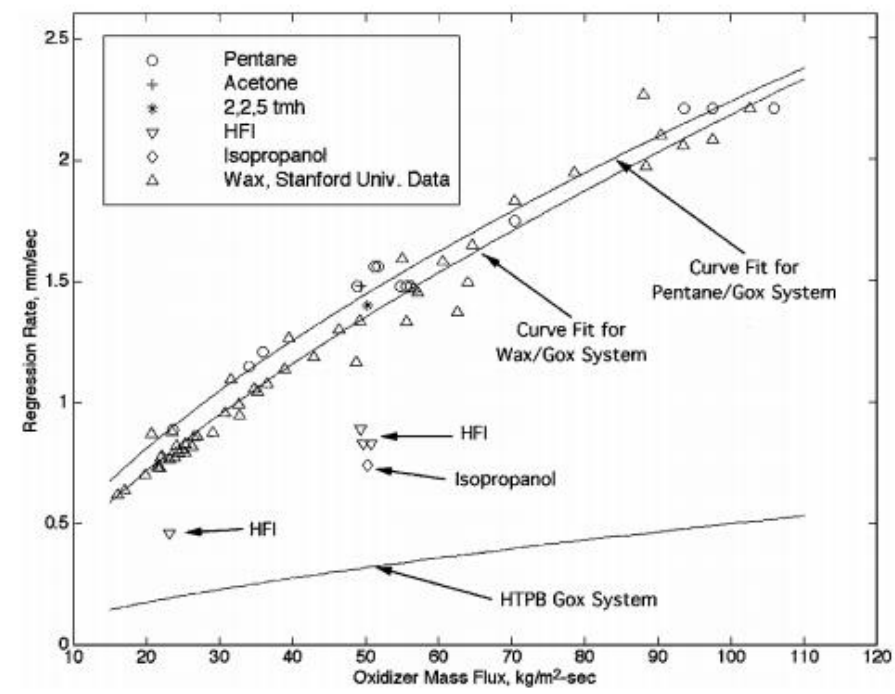

Figure 17. Regression rate data for O2 hybrid engines for various solid fuels as a function of oxidizer mass flux [32]

The above chart allows for determination of a fuel regression rate, the speed at which the solid fuel regressions towards the chamber walls, as well as oxidizer mass flux for a chosen design o/f and chamber geometry. This burn velocity $\dot{r}$ for a cylindrical combustion chamber port can be linked to fuel flow rate using equation 2.11 below for a given chamber diameter $\mathrm{D}$, length $\mathrm{L}$, and fuel density $\rho_{\text {fuel }}$.

$$
\dot{m}_{f u e l}=\dot{r} \pi D L \rho_{\text {fuel }}
$$

The total mass flow rate through the engine is now simply the sum of the oxidizer and fuel mass flow rates. This parameter along with a chosen nozzle throat area to allow for a reasonable oxidizer supply and chamber size allow for proper prediction of the chamber pressure. With a chamber pressure now accounted for, the operation of the hybrid system now becomes analogous to that of the cold flow system used in this work, allowing for establishment of a hot, combusting free jet. The obvious difference with this combusting case is a much high static temperature at the nozzle exit as hot combustion gases are expanded to supersonic speed rather than room temperature 
air. Given a known pressure ratio and combustion temperature, the expected isentropic exit temperature of the combustion gases can be computed as presented in equation 2.12.

$$
T_{e}=T_{o}\left(\frac{P_{e}}{P_{o}}\right)^{\frac{k-1}{k}}
$$




\section{Experimental Methods}

\subsection{Gas supply system}

The experimental work in this study were completed in LSU's Engineering Research and Development (ERAD) building, a shared lab building which shares both multidisciplinary laboratory and office space. The laboratory is located on the first floor with convenient access to ventilation, water supply, as well as compressed air supply. Figure 18 below shows the compressed air system used to supply all laboratories in ERAD, and which was used over the course of the test program. The system consists of an Atlas Copco GA315 compressor feeding into a 2560 gallon accumulation tank through a Zander KN32-EC industrial dryer. The accumulation tank has a maximum allowable working pressure (MAWP) of 200psig at 400, and nominally operates at 150psig and ambient temperature.

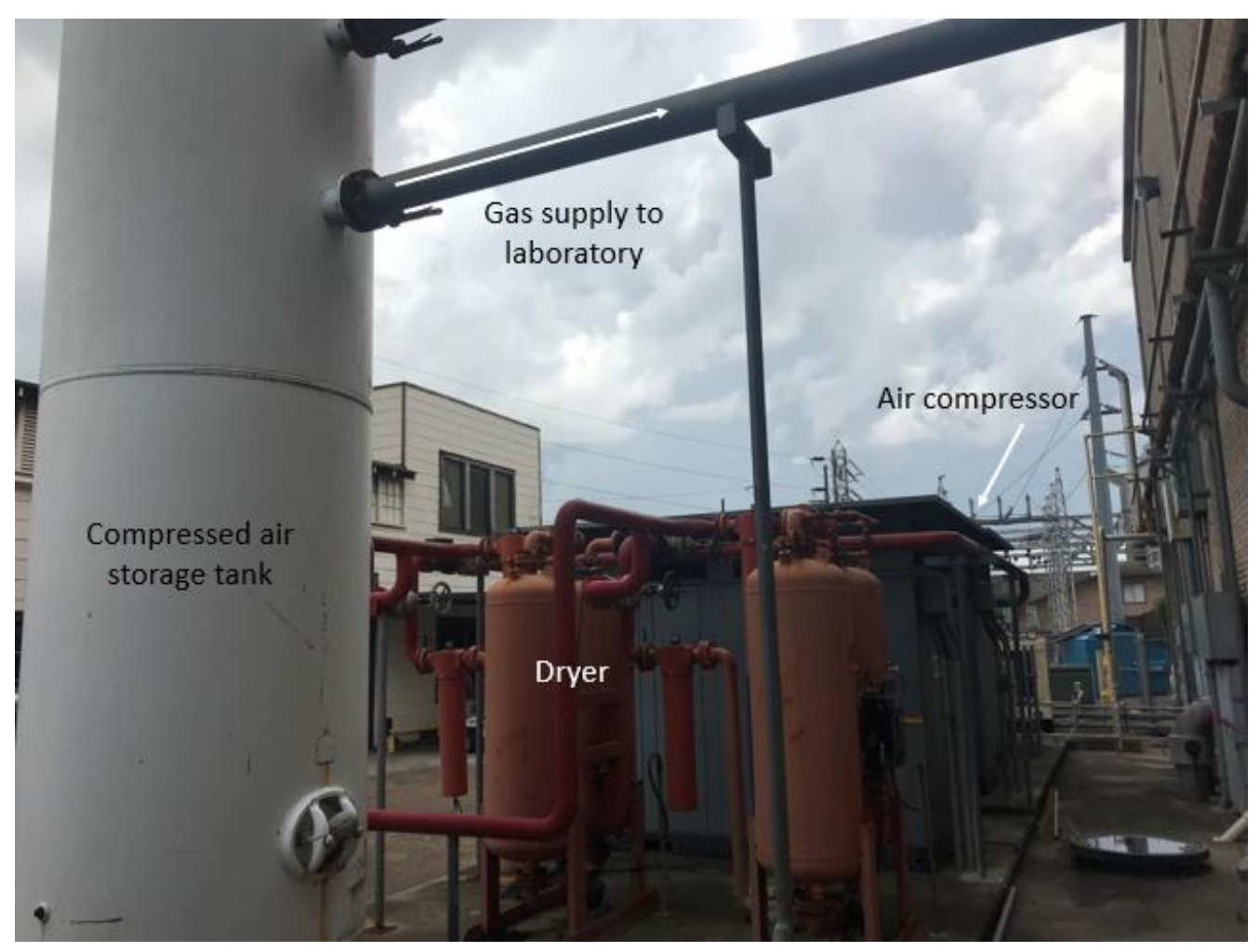

Figure 18. Laboratory compressed air supply system 
This gas supply system feeds into the lab space and can be accessed via a 1" schedule 40 pipe. At maximum nominal operating pressure, the compressed air system can deliver $~ 70$ SCFM of air to the laboratory. Given this value of maximum mass flow rate of air to the laboratory, a nozzle geometry was designed to deliver an overexpanded free jet of air to the experimental setup at a design Mach number of 3. Figure 19 below shows this nozzle geometry with a throat diameter of $0.2 \%$ and an exit area ratio of $\frac{A_{e}}{A^{*}}=3.61$. The nozzle was chosen to have a conical contour for simplicity of manufacture, though this increases the risk of flow separation for an overexpanded flow[33].
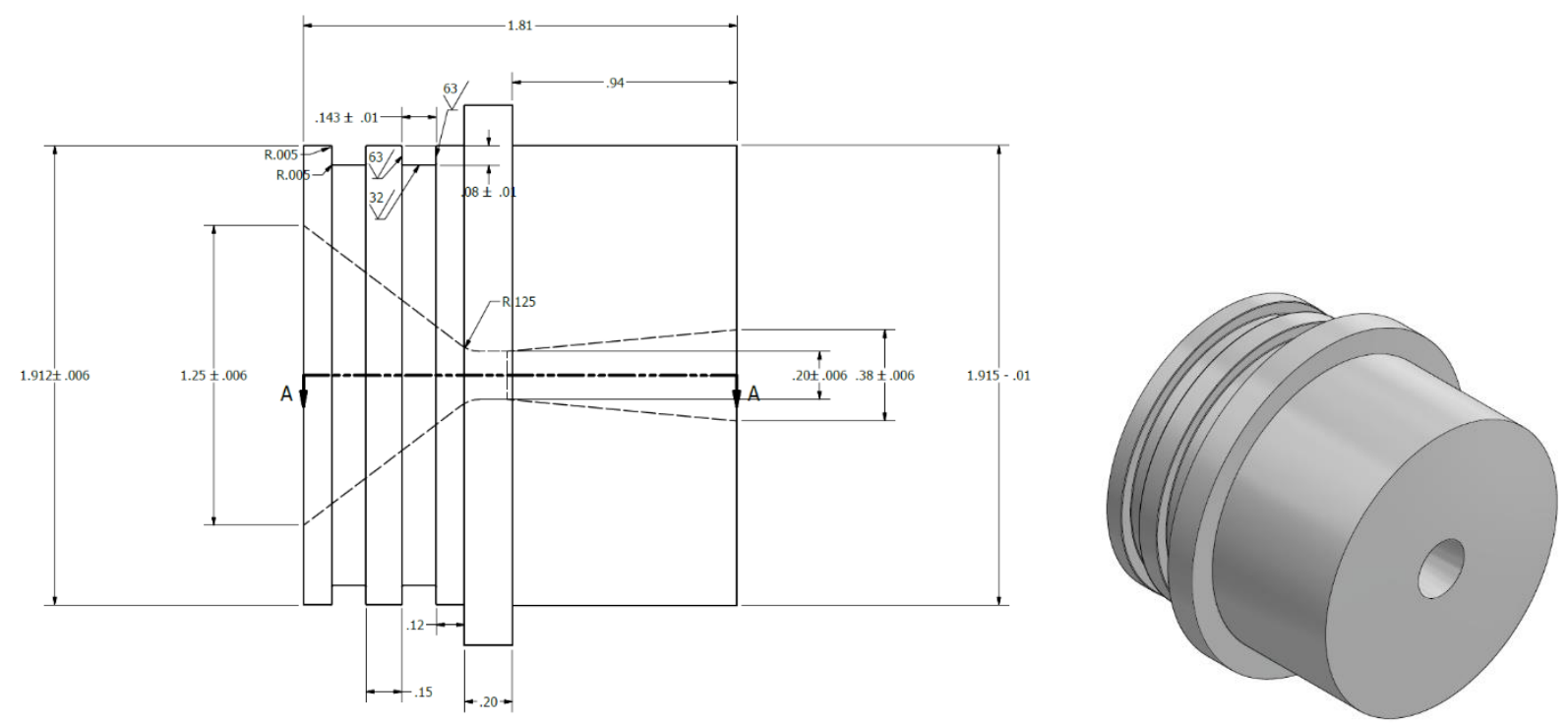

Figure 19. Left: Dimensioned air nozzle drawing, Right: Air nozzle isometric view

The flowrate of air into the experimental setup is measured using a Dwyer VFC-123 rotameter with the pressure correction in the reading being accounted for using an analog static pressure gauge placed downstream of the rotameter. The air supply is transported from the 1" supply line to the experiment stand using $3 / 8$ " nylon tubing and enters the air testing chamber through a $3 / 8$ " schedule 40 pipe cross allowing for introduction of temperature and pressure measurement hardware upstream of the test chamber. A type-k thermocouple and an AST 4000 
series $4-20 \mathrm{~mA} 0-250 \mathrm{psig}$ pressure transmitter are used to measure the static temperature and pressure of the air flow prior to entering the chamber, respectively, and are indicated in Figure 24.

\subsection{Water supply system}

To precisely control water flow into the experimental setup, a pressurized cylinder rated for 190psig MAWP with a dip tube is used to supply the water. Pressurized air from the compressed air accumulation tank is used to provide pressurant for the cylinder, allowing for this pressure to be finely controlled. Figure 20 below shows this storage cylinder and the location of the water fill and outlet ports and the gas pressurization inlet.

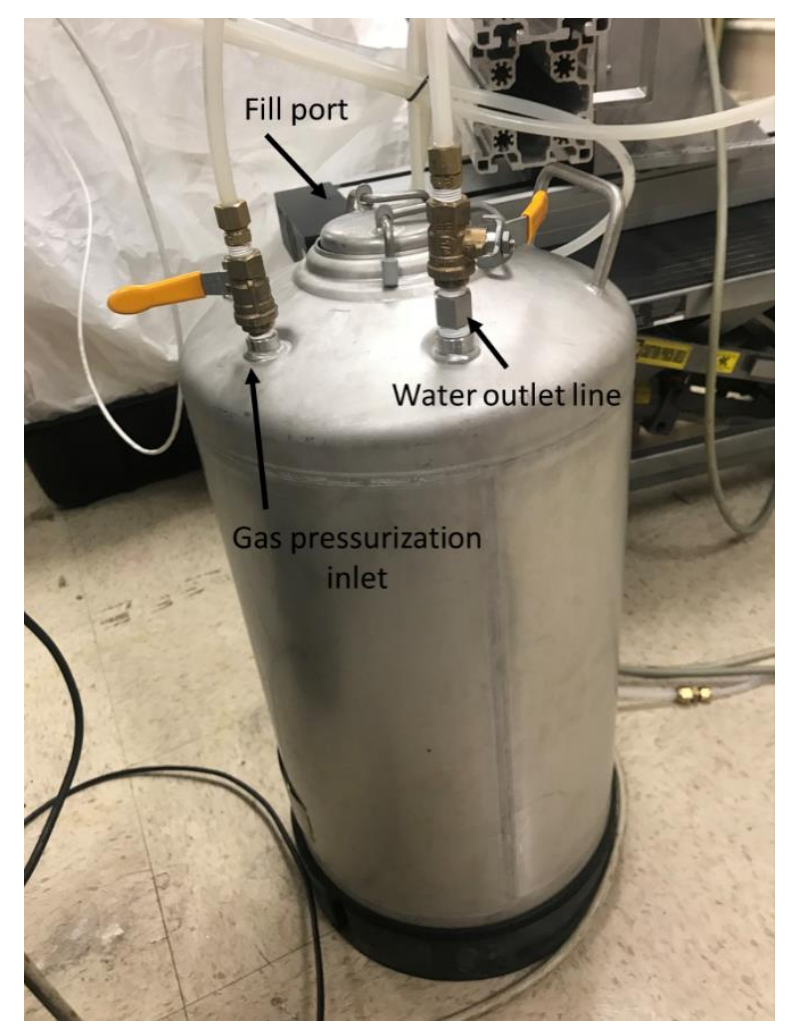

Figure 20. Water pressurized storage cylinder

The pressurization air is dried using a Wilkerson X03-04-U00 dryer and regulated using an Alemite 7604-1 regulator rated to deliver up to 300psig regulated gas pressure. The water is transported from the pressurized cylinder to the test stand through $3 / 8$ " nylon tubing with flow 
being controlled by a LabVIEW controlled 3/8" NPT Granzow H3U29-00Y electrical solenoid valve. Flowrate through the tubing is measured using an Omega FTB-2006 hall effect flowmeter powered by the $5 \mathrm{~V}$ output on the data acquisition unit (DAQ). The water is then fed into a $3 / 8$ " schedule 40 pipe cross where water static temperature and pressure are measured using a type-K thermocouple and 0-5V output Omega PX309-300G5V pressure transducer, respectively, before exiting through the water nozzle. The water nozzles are a sharp-edge orifice type nozzle with $0^{\circ}$ spray angle and a small internal baffle to aid in generation of a uniform flow through the nozzle exit. Three different water nozzle sizes were investigated in this work with exit diameters of 0.03 " $(0.76 \mathrm{~mm}), 0.04 "(1.02 \mathrm{~mm})$, and $0.05 "(1.27 \mathrm{~mm})$. Figure 21 below shows the flow rate provided by each nozzle used as a function of nozzle back pressure.

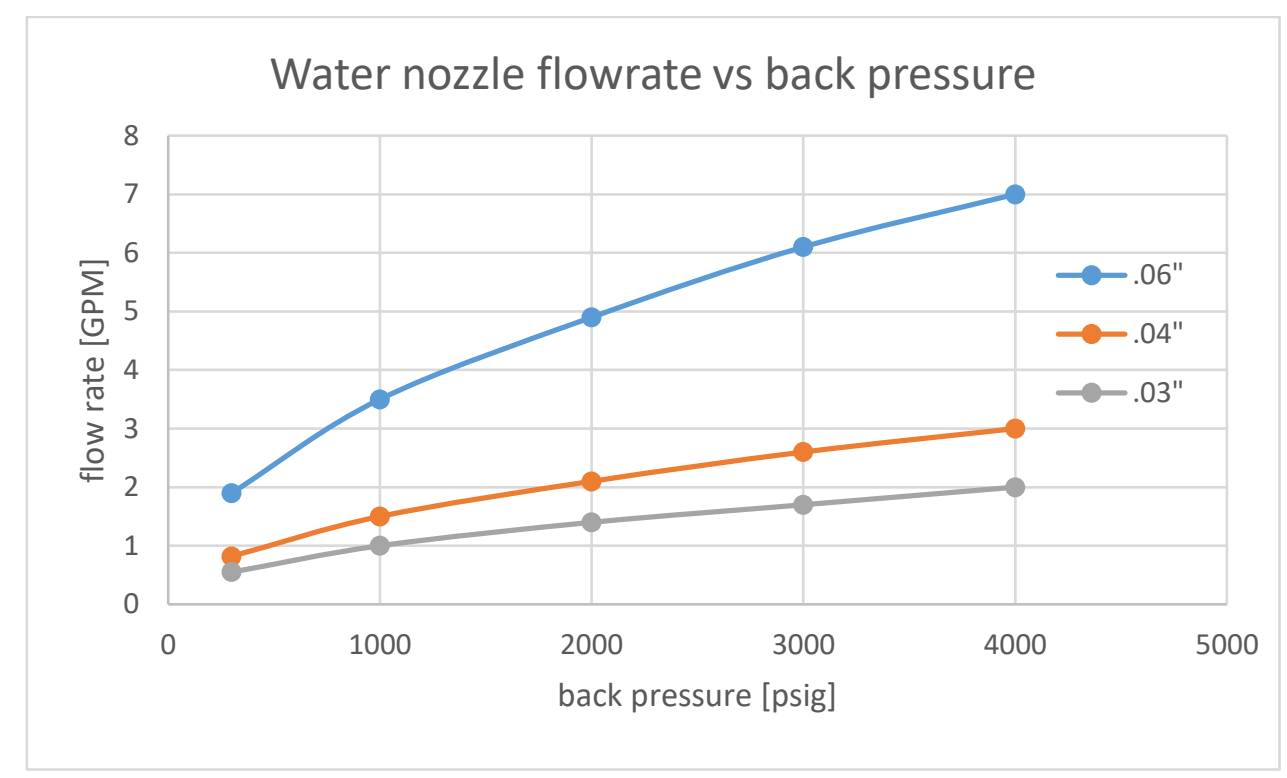

Figure 21. Water nozzle flow rate as a function of back pressure

Figure 22 below shows schematically the flow paths for both gas and liquid phase flows as well as the relative location of both compressed air and water nozzles in the experimental setup. 24V DC power is provided to the flowmeter and solenoid valve via a Traco Power TBLC 75-124 
75W power supply, with the power to the solenoid being controlled using a Crydom DC60S3 solid state relay, triggered by a $5 \mathrm{VDC}$ digital signal from the DAQ.

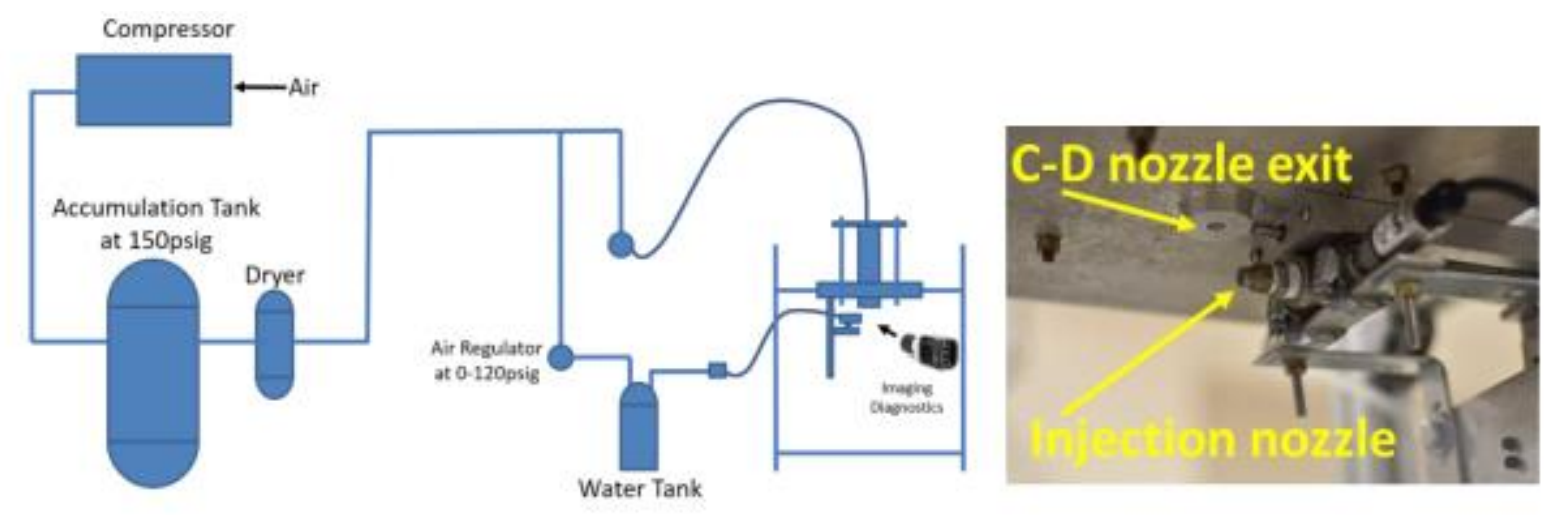

Figure 22. Left: fluid flow path diagram, Right: Experimental setup with compressed air and water nozzles

Output from all thermocouples are read using a National Instruments NI-9211 thermocouple input device and all other control digital control signals and data are recorded using a National Instruments USB X Series Multifunction DAQ. Appendix A shows images of each of these pieces of hardware.

To interface with the stand and record data from the measurement devices properly, a LabVIEW interface was created as shown in Figure 23. Following a user input of the ambient conditions at the time of testing, including ambient temperature, pressure, and air flow rate, the user is prompted to begin running the control loop for the stand. Once the user commands the flow of water to begin, the LabVIEW program begins recording data for static temperature and pressure for both gas and water inlets. The frequency reading generated by the hall effect flow meter is converted to an equivalent flow rate in gallons per minute (GPM). The program also allows for the termination of testing in event of an emergency or hardware failure on the stand. All the data are written to spreadsheet files for post-processing. 


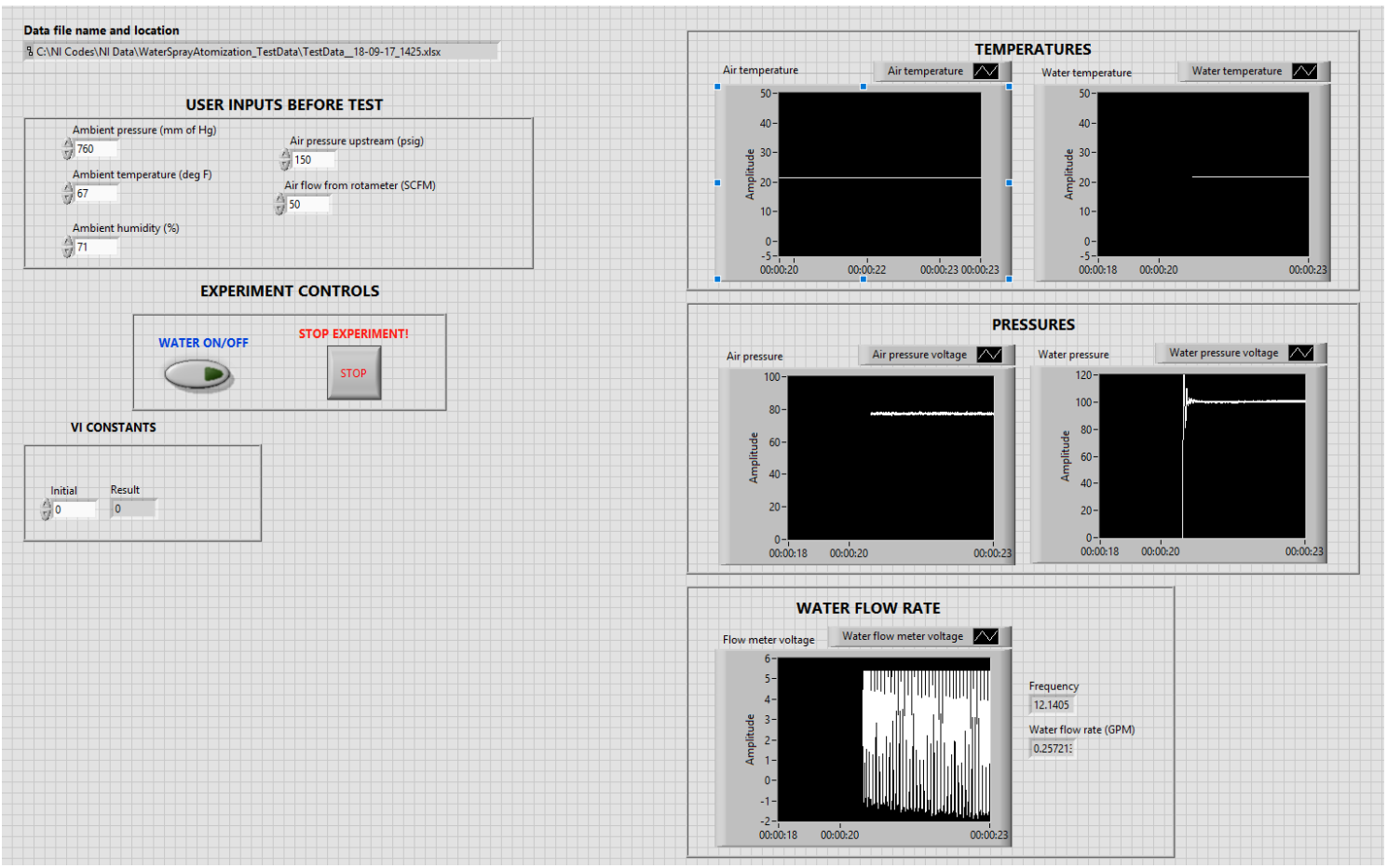

Figure 23. Screenshot of LabVIEW interface

Figure 24 below shows views of the compressed air inlet and instrumentation ports (left) and water injection and instrumentation ports (right). 


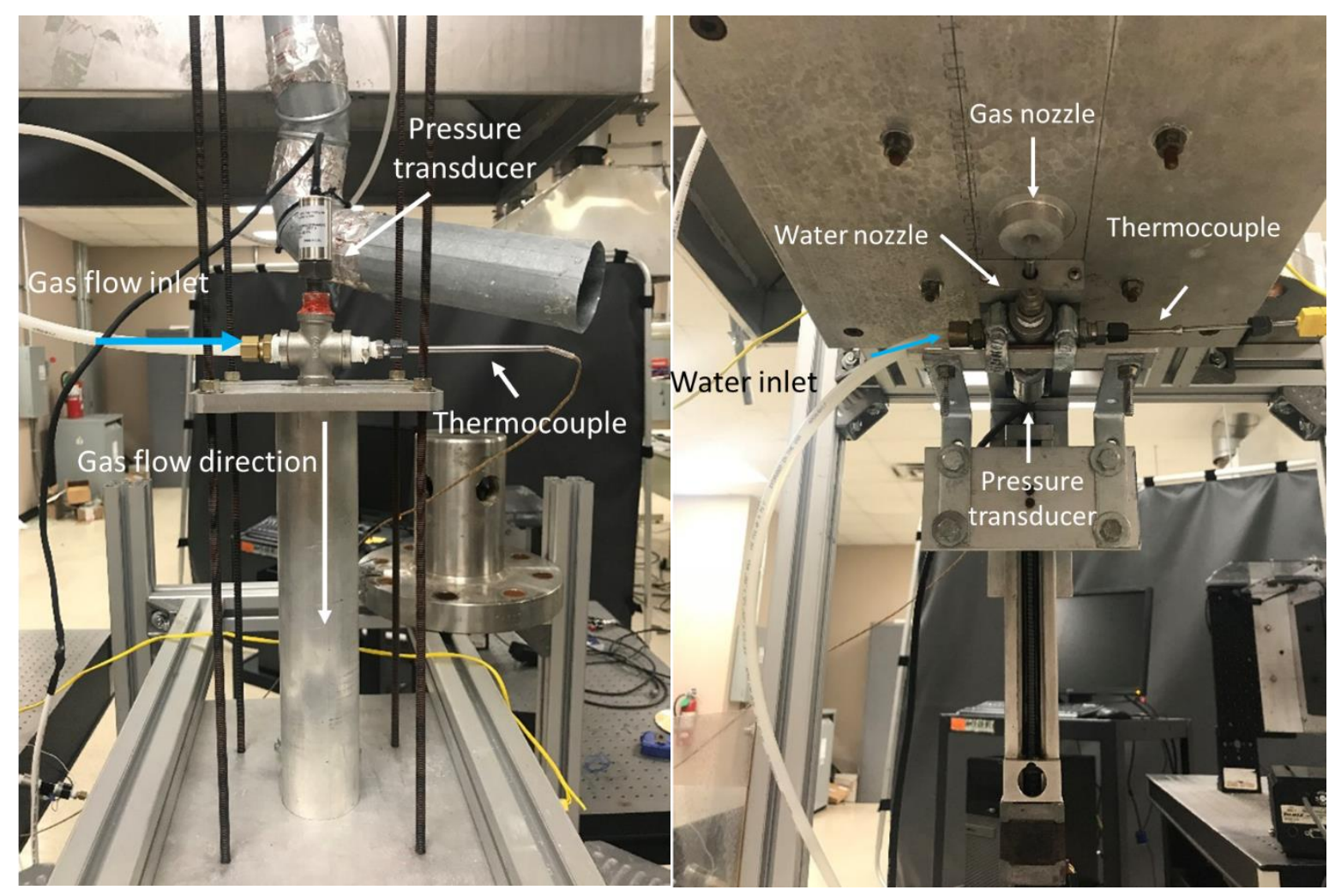

Figure 24. Left: Compressed air inlet and chamber, Right: Vertically actuated water nozzle

\subsection{Diagnostics}

As the experimental setup involves a compressible gas phase flow it is important that any diagnostic tools used to examine flow behavior be non-intrusive as not to alter the natural shockwave structure in the gas phase. As such, several laser-based and optical instruments were used to examine the flow behavior without introducing any disturbances. These diagnostic tools will be introduced next.

\subsubsection{Droplet size and velocity measurement}

Droplet velocity and size in the liquid spray region is of key interest in this work. Quantitative information regarding these parameters would serve directly as validation data for the CFD results. There are several laser diagnostic techniques able to measure spray droplet size and velocity simultaneously in the near field (dense spray) and far-field (more dilute spray). Popular techniques include Phase Doppler Particle Anemometry (PDPA), Interferometric Particle Imaging 
(IPI/ILIDS), holography, and high-resolution imaging []. Droplet velocity by itself can be measured using Particle Image Velocimetry (PIV). PDPA was chosen as the diagnostic method of choice for obtaining these measurements as it has several advantages over other techniques for this applications including:

- PDPA is capable of operating in regions of dense spray and has been extensively used in previous studies involving spray characterization.

- It is based on light-scattering interferometry and is calibration free.

- Although a point measurement, the components are easily set up on an automated traverse that can obtain measurements on a 2-D or 3-D grid.

- The compact detector is capable of providing 2-component velocities as required for this application.

Figure 25 below shows schematically the operation of the classic PDPA system in use in LSU's EPL. The light sources from the probe which intersect at a fixed measurement volume location is captured by a detector carefully chosen angle relative to the probe which focus the light at the intersection to be received by the system's photomultipliers. The signal received by the photomultipliers converts the light intensity to an electrical signal which can be ready by the burst spectrum analyzer. 


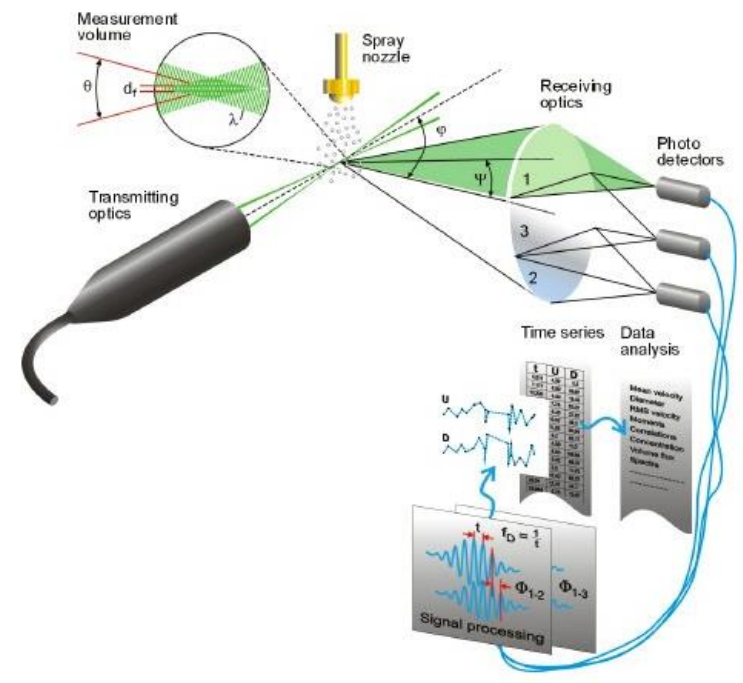

Figure 25. Schematic of PDPA operation [35]

The velocity of a particle passing through the measurement volume is linearly related to the doppler burst detected. Equation 3.1 below shows the calculation of this velocity as a function of the physical parameters of the laser system.

$$
U=\frac{\lambda}{\sin \left(\frac{\theta}{2}\right)} f_{D}
$$

Similarly, equation 3.2 below shows computation of the particle diameter D of a particle passing through the measurement volume. The particle size is measured directly from a phase shift in the doppler signals.

$$
\Phi=\frac{-2 \pi D}{\lambda} \frac{n_{r e l} \sin \theta \sin \psi}{\sqrt{2(1+\cos \theta \cos \phi \cos \psi)\left(1+n_{r e l}^{2}-n_{r e l} \sqrt{2(1+\cos \theta \cos \psi \cos \phi)}\right)}}
$$

A two-component classic PDPA setup is used to measure water droplet sizes and velocities in the spray [35]. A continuous Argon ion laser (Spectra-Physics Stabilite 2017) is used which can emit $1.5 \mathrm{~W}$ at $476.5 \mathrm{~nm}$ and $2 \mathrm{~W}$ at $514.5 \mathrm{~nm}$. The green light at $514.5 \mathrm{~nm}$ is intended to measure the $\mathrm{U}$ component of velocity (vertical component) while the violet light $(476.5 \mathrm{~nm})$ is intended for 
$\mathrm{V}$ component of velocity (one of the components in the horizontal plane). In this work, only the $\mathrm{U}$ component of velocity has been measured due to difficulties in obtaining reliable data using the less powerful violet wavelength. Table 1 below presents the parameters used when operating the PDPA system. A classic PDPA receiver and photomultipliers was used along with the newest version of Dantec's PDPA analysis software and burst spectrum analyzer (BSA).

Table 1. PDPA system parameters

\begin{tabular}{|c|c|c|c|}
\hline \multicolumn{2}{|c|}{ Parameter } & Droplet Data & Units \\
\hline \multicolumn{2}{|c|}{ Scattering angle } & 60 & Degrees \\
\hline \multirow{3}{*}{$\begin{array}{l}\text { Probe } \\
\text { volume }\end{array}$} & $\mathrm{x}$-dimension & 0.1943 & $\mathrm{~mm}$ \\
\hline & y-dimension & 0.1941 & $\mathrm{~mm}$ \\
\hline & z-dimension & 4.091 & $\mathrm{~mm}$ \\
\hline \multicolumn{2}{|c|}{ Number of fringes } & 35 & \\
\hline \multicolumn{2}{|c|}{ Fringe spacing } & 5.422 & $\mu \mathrm{m}$ \\
\hline \multicolumn{2}{|c|}{ Beam diameter } & 1.35 & $\mathrm{~mm}$ \\
\hline \multicolumn{2}{|c|}{ Beam separation } & 38 & $\mathrm{~mm}$ \\
\hline \multicolumn{2}{|c|}{ Transmitter focal length } & 400 & $\mathrm{~mm}$ \\
\hline \multicolumn{2}{|c|}{ Receiver focal length } & 600 & $\mathrm{~mm}$ \\
\hline \multicolumn{2}{|c|}{ Maximum particle diameter } & 180 & $\mu \mathrm{m}$ \\
\hline
\end{tabular}

To precisely place the measurement volume of the PDPA probe in the spray, a 2-axis ISEL traverse system was used, allowing for computerized control of the positioning via Dantec's PDPA software. Figure 26 below shows the PDPA system integrated with the experimental setup with the probe and receiver setup in the refraction-receiving mode on the ISEL traverse. 


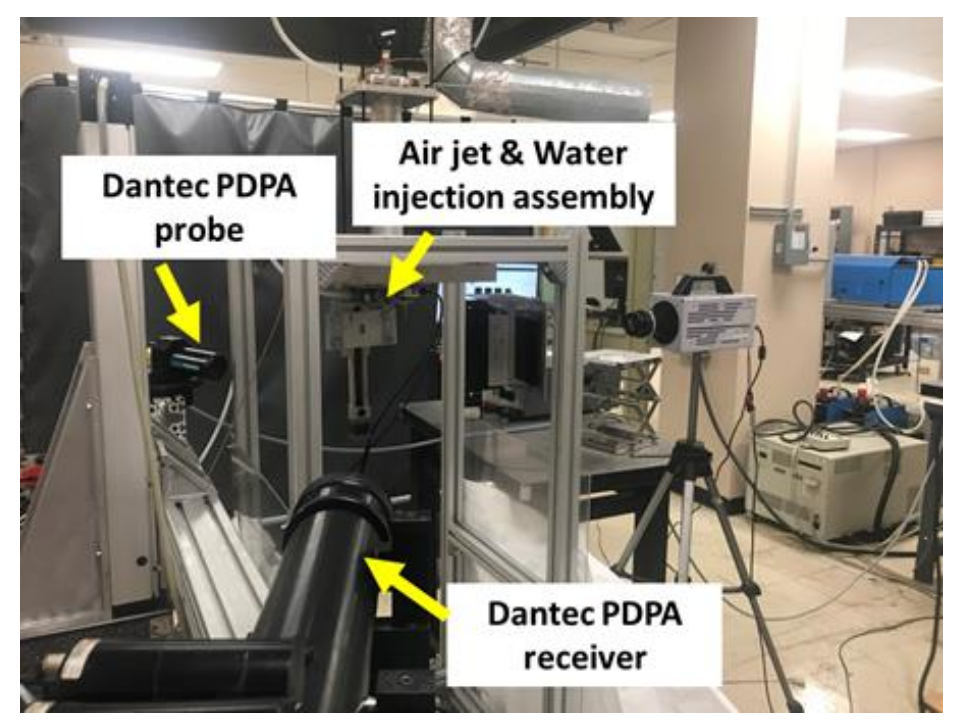

Figure 26. PDPA system and experimental setup

While Figure 26 shows the PDPA system mounted only on a 1-D traverse the system has recently been upgraded to move in 2-D to facilitate future work. Figure 27 below shows the $2 \mathrm{D}$ traverse in use in the laboratory, allowing for future automated control over 2D positioning of the measurement volume in the liquid spray.

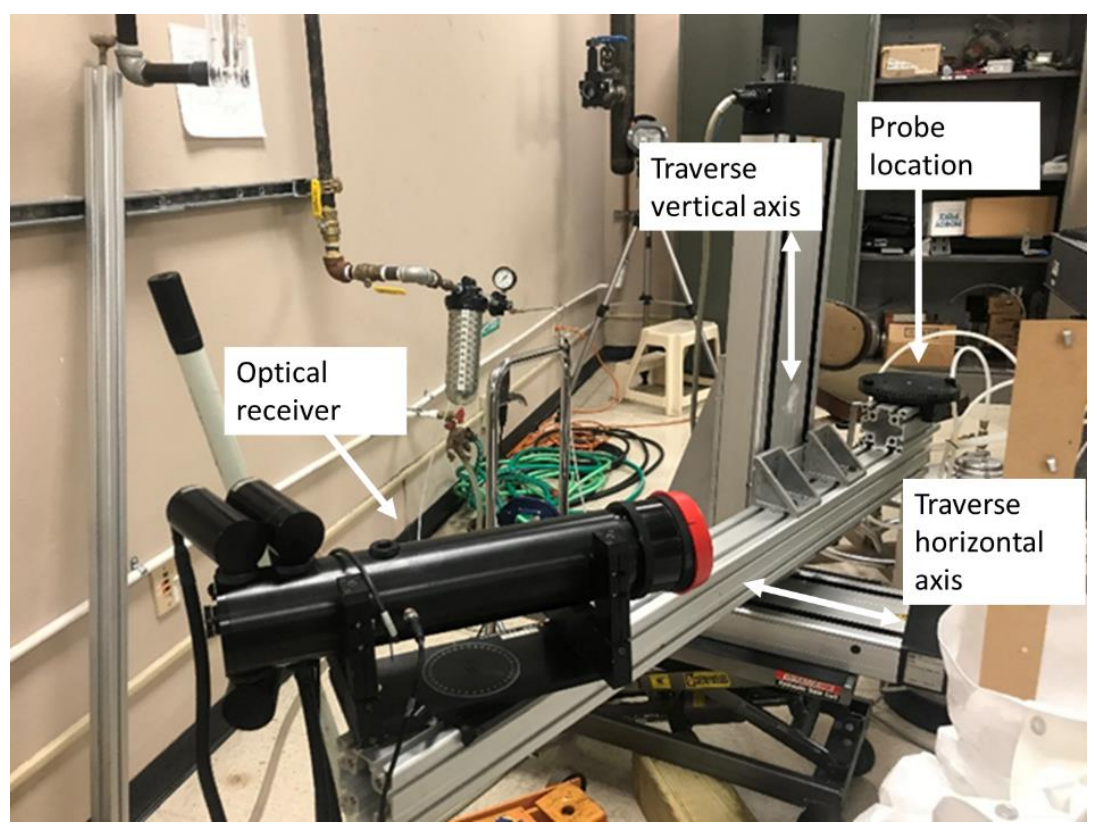

Figure 27. PDPA system on $2 D$ traverse 
The output from the software is typically in the form of histograms showing droplet size and velocity distribution over the measurement duration. To obtain reliable and fast input data rate using the PDPA system it is very important to properly threshold the sensitivity of the photomultipliers. During alignment of the system and initial setup prior to full test runs, a representative spray is generated using a household humidifier, a source that has a generally known and steady approximate particle size and velocity. As this fast data rate becomes very important to obtain reliable data, another test run is done with the spray of interest before acquiring data and the photomultiplier sensitivities and gains are again corrected.

\subsubsection{Spray geometry}

The overall spray structure provides a means to compute quantities such as jet penetration and provides dynamic information regarding the breakup process, which can be used to analyze the breakup regimes as indicated in Figure 13. In this work, spray morphology was examined using a high-speed camera and two different types of light sources. These approaches are discussed next.

\subsubsection{Laser sheet illumination}

In this approach, a high speed camera (Photron SA-3) was used with a laser sheet formed at the mid-plane of the air and water nozzles to gain insight into the flow behavior. The experimental setup is shown in Figure 28. A laser sheet is generated using the output from a pulsed Nd:YaG laser (New Wave Solo PIV) combined with a cylindrical lens. The laser provides 120 $\mathrm{mJ} /$ pulse at $532 \mathrm{~nm}$ wavelength with a $15 \mathrm{~Hz}$ pulse rate. The sheet is imaged using a Photron SA3 high speed camera arranged at right angles to the laser sheet. The camera is triggered using the synchronization pulse generated by the laser. The laser pulse width is 3-5 ns while the images are

acquired at 60 frames per second giving a time scale of $17 \mathrm{~ms}$. The continuous $514.5 \mathrm{~nm}$ 
wavelength PDPA probe is also used as a laser source to obtain higher speed video to examine the penetration distance and dynamic behavior of the liquid breakup process.

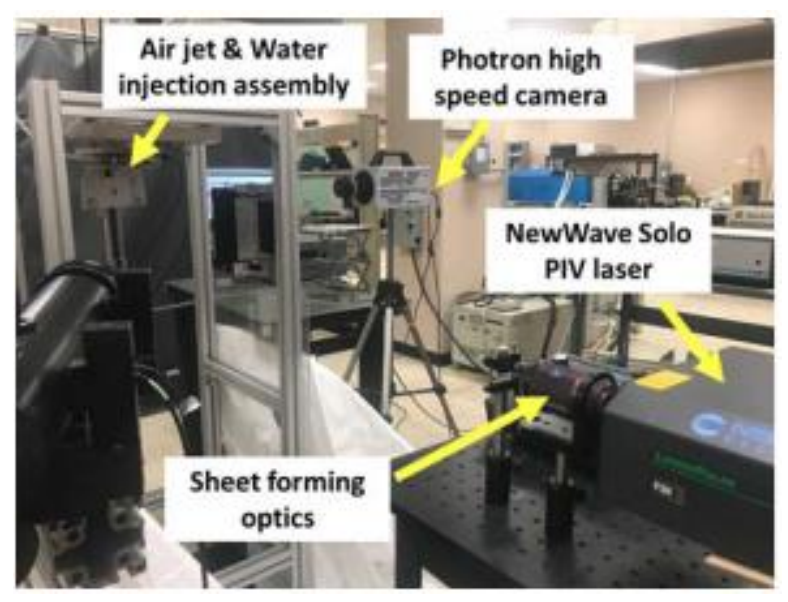

Figure 28. Experimental setup for the laser sheet imaging

\subsubsection{Volume illumination}

Along with the laser sources used to take a sliced view at the inner spray morphology, volume illumination is used along with the high-speed camera to examine global spray penetration and behavior. This technique further allows for well-resolved views of the initial phase of coherent liquid jet breakup. Figure 29 below shows the laboratory setup with a 250W halogen lamp at a distance of 3 meters and $\sim 15^{\circ}$ off-angle from the high-speed camera-viewing plane is used as a diffuse light source of refracted light. This setup has been primarily used to obtain the penetration height of the water jet into the supersonic crossflow. 


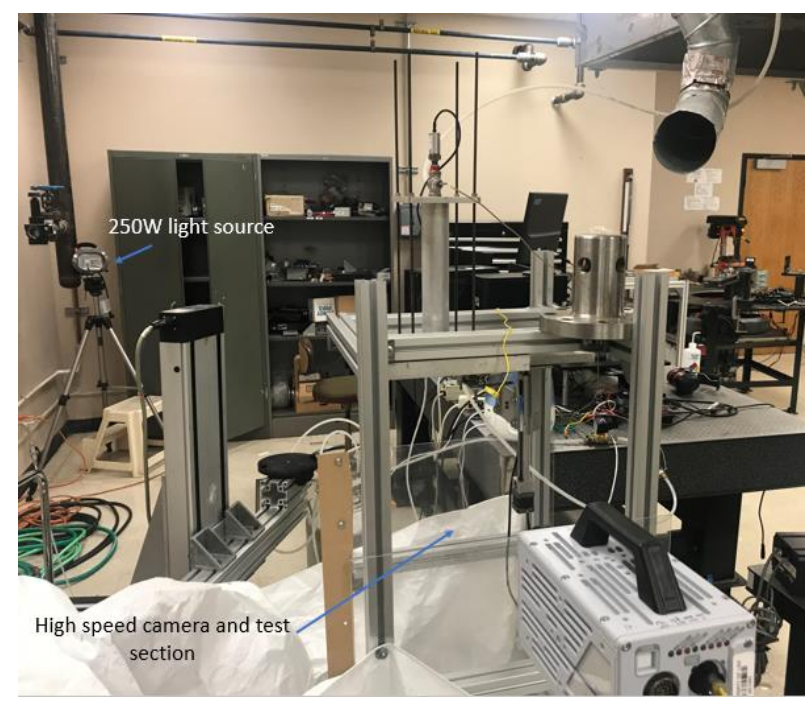

Figure 29. Light source orientation for high speed photography

The Photron SA-3 camera is operated at 2,000 frames per second with a resolution of $1024 \times 1024$ pixels for all jet penetration data taken in this work. Images were acquired for a total elapsed time of $0.5 \mathrm{~s}$. The camera can also be operated at a maximum of 120,000 frames per second albeit with a reduced resolution. To examine the dynamics of the initial liquid breakup region, an increased frame rate of 40,000 frames per second was used with a lower resolution.

\subsubsection{Shock structure}

The shock structure at the exit of the overexpanded air nozzle is as shown in Figure 11. Examining the changes induced in this shock structure as a result of liquid jet injection and correspondingly the effect of jet injection location in the shock structure on the resulting spray morphology are important goals of this work. Achieving these goals requires the ability to visualize the shock structure, which is essentially composed of a series of interfaces caused by density variations across the flow structures. Various techniques have been developed to image shock structures with the most popular ones involving shadowgraphy and Schlieren imaging. In this work, a form of Schlieren imaging called focusing color Schlieren (FCS) photography was used to examine the shockwave structure in the gas phase and its changes due to the introduction of the 
liquid stream and spray. Similar to traditional Schlieren photography, FCS allows for the capture of areas of density change in a test area. The density differences are illuminated or darkened depending on a change in their refractive index. Figure 30 below shows a traditional Z-Type Schlieren photography system. Here, a collimated light source which has passed through a test section of interest is focused to a point using a parabolic mirror, allowing for changes in refractive index to be measured as the light from the test section interferes with the razor edge.

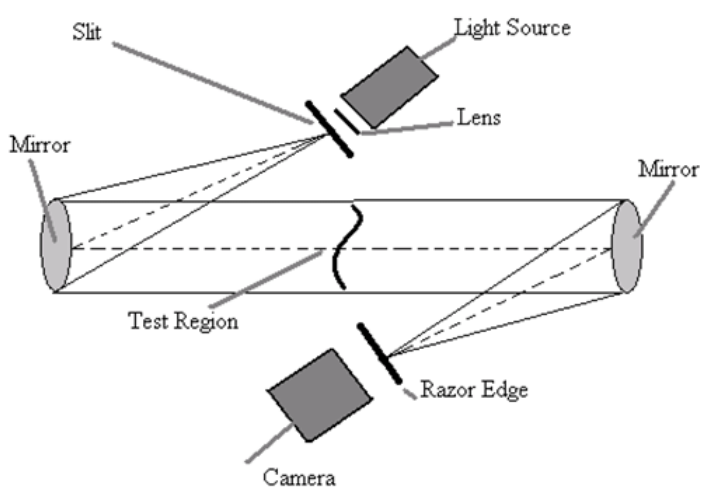

Figure 30. Traditional z-type Schlieren setup [37]

Focusing color schlieren allows for the assignment of color to a directional change in refractive index by using a carefully arranged multi-color light source grid. This densely populated source grid is matched by a cutoff grid in the optical section of the instrument where distinct cutoff squares act as knife edges would in traditional Schlieren photography to capture the refracted source light. Figure 31 below shows schematically the FCS instrument with the TFT source panel and imaging lens containing the previously discussed source grid and cutoff grid. 


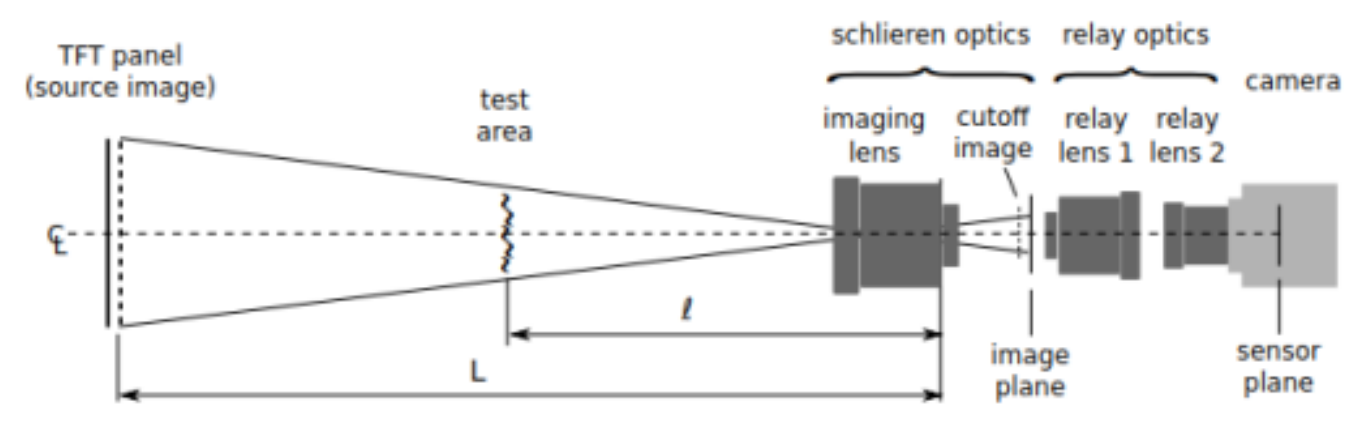

Figure 31. Schematic diagram of focusing color schlieren system[35]

The ability of this system to focus on a specific thin focal plane in the test section allows for elimination of some of the inaccuracies that would come up with attempting to examine a crosssection of a conical free supersonic gas jet. The traditional Schlieren image is a line-of-sight integrated image. The FCS implementation allows for better resolved slicing of the largely axisymmetric gas jet of interest. The system utilized in this work was developed by Dr. Ingmar Schoegl's group at LSU and allowed for a simple integration into the experimental setup. The details of the FCS imaging technique and setup are provided elsewhere [35]. A Nikon D5600 DSLR camera with a $50 \mathrm{~mm}$ focal length lens set at f/1.4 with a 60 frame per second video resolution of 1920x1080 was used for all video taken using the FCS system. The lens and aperture chosen were used to integrate well with the existing optics on the system and to ensure the thinnest imaging plane was used to minimize integrated optical errors.

\subsection{Proper orthogonal decomposition}

To aid in examining the dynamic behavior of the liquid primary breakup mode, images recorded using the high-speed camera were post-processed using a technique called proper orthogonal decomposition (POD). The aim of this technique is to decompose the complex dynamic breakup process of the liquid into a set of simpler orthogonal modes which can be superimposed to recover the more complex flow. The idea being that each of these orthogonal modes helps reveal 
a dominant overall mode of motion. The method used in the current work is that of the method of snapshots, developed in [38]. In order to properly image the dynamic behavior of the flow including evidence of surface wave motion, it is very important to be able to "freeze" the flow or record at a frame rate fast enough to properly resolve the motion.

A brief discussion of the method of calculation for the orthogonal modes is presented next. For a detailed description of the technique, the reader is referred to the work by Lumley [39] or Arienti [38]. Equation 3.1 below shows the general form of the equation used to determine a minimum frame rate $f$ necessary to capture the flow's dynamic motion. This frequency should be the inverse of the aerodynamic characteristic time, or the amount of time needed for a fluid element to travel across a characteristic length of the flow $d_{o}$. Generally, $d_{o}$ is taken to be the nominal size of the liquid jet. The frequency is also related to the fluid densities and gas flow speed $u_{\infty}$. To properly avoid aliasing in the imaging process the sampling rate should be $f_{s}>2 f$.

$$
f=\frac{1}{t^{*}}=\frac{u_{\infty}}{d_{o}} \sqrt{\frac{\rho_{\text {gas }}}{\rho_{\text {liquid }}}}
$$

For the method of snapshots, the goal is to generate a set of orthogonal basis functions which span a collected ensemble of snapshots. In the case of our photographic data, these functions will seek to display modes that capture the maximum amount of energy in the flow as pixel intensity values with respect to the average flow behavior. For a set of $\mathrm{N}$ images, each of size $n \times m$ pixels, the distance between any two images $x_{p}$ and $x_{q}$ is defined as shown in equation 3.2 below where $x^{j, k}$ represents the $\mathrm{j}^{\text {th }}$ row and $\mathrm{k}^{\text {th }}$ column element of the matrix $x$.

$$
<x_{p}, x_{q}>=\sum_{j}^{m} \sum_{k}^{n} x_{p}^{j, k} x_{q}^{j, k}
$$


Given a set of $\mathrm{N}$ images, one can compute up to $\mathrm{M}=\mathrm{N}-1$ orthogonal modes which span the set conveniently as long as $n \cdot m \gg N$. First, one must compute the mean of the image set $\left(\phi_{o}=\right.$ $\frac{1}{N} \sum_{i}^{N} x_{i}$ ), which also serves as the $0^{\text {th }}$ orthogonal mode, simply representing an average pixel intensity for the image set. Equation 3.3 below demonstrates computation of the correlation matrix $K$ representing the difference between two snapshots after subtracting the background. This step is important as the subtraction allows for isolation of dynamic behaviors in the image set from stationary background objects. This allows for simple image capturing methods to be used as care need not be taken to eliminate stationary background objects.

$$
K^{i, j}=\frac{1}{N}<\tilde{x}_{i}, \tilde{x}_{j}>
$$

Using this definition of the correlation matrix $K$, one can now compute proper orthogonal modes $\phi_{r}$ for $r \leq N$ by solution of the linear eigenvalue problem $K v_{r}=\lambda_{r} v_{r}$ where $v_{r}$ and $\lambda_{r}$ are the orthogonal eigenvectors and real and non-negative eigenvalues of the correlation matrix $K$. Equation 3.4 below shows the computation for the mode $\phi_{r}$ for a set of $\mathrm{N}$ images.

$$
\phi_{r}=\frac{1}{\sqrt{N \lambda_{r}}} \sum_{i=1}^{N} v_{r i} \tilde{x}_{i}
$$

The eigenvalues $\lambda_{r}$, corresponding to each mode $\phi_{r}$, measures the contribution of that mode to the overall system dynamics. Once computed, the modes $\phi_{r}$, are 2-D matrices of size $m$ $x n$ which can be plotted. Different orthogonal modes link to different physical features of the jet and can provide a deeper understanding of the jet structure. While a large number or orthogonal modes can be computed ( $\mathrm{N}-1$ for $\mathrm{N}$ images), the overall system dynamics can be computed by just the first few modes which contribute to much of the energy (or pixel illumination) and whose eigenvalues are sufficiently separated from those of the remaining modes. A good illustration of 
the ability of the POD analysis to identify jet structures by separating the orthogonal modes is illustrated by Arienti as shown in Figure 32. Figure 32(a) shows a snapshot of a laminar liquid jet in time. Figure 32(b-d) show the first four calculated modes which capture $82 \%$ of the energy. Figure 32 (b-c) show the presence of a column flapping mode (jet moving back and forth longitudinally) while Figure 32(d-e) show the presence of traveling waves which are spaced uniformly. These structures would otherwise be difficult to detect in the composite jet structure of Figure 32(a). The preceding POD analysis will be utilized in this work to analyze the liquid jet structure as captured by high speed images.

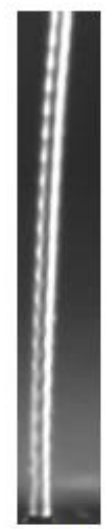

(a)

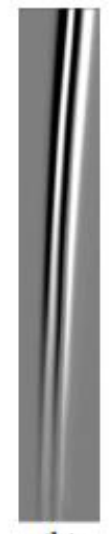

(b)

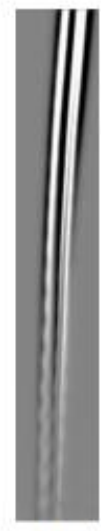

(c)

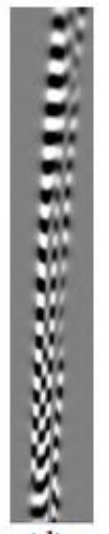

(d)

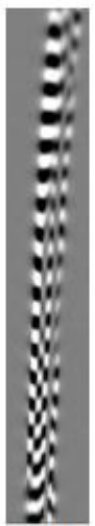

(e)

Figure 32. POD decomposition of a laminar jet at low Weber number. (a) Jet snapshot (b-d) First four orthogonal modes [38]

\subsection{Hybrid rocket engine design}

Ongoing work is in progress to develop a hybrid rocket system to deliver a combusting supersonic free jet to better approach testing conditions present at SSC. While the theory of the rocket design was presented in a previous section, the design and construction progress on this system will now be presented. An oxidizer rich gaseous oxygen (GOx) and HTPB core burning hybrid rocket design was chosen to operate as a bench-scale $(<15 \mathrm{lbf}$ thrust) engine used to transition from cold flow testing to future hot flow testing. This engine is also intended to be 
developed for use in future laboratory projects. The engine is intended to be ignited using a traditional pyrotechnic ignition source. Gaseous oxygen flow is controlled using a single ball valve and check valve. Figure 33 below shows schematically the layout of the hybrid rocket system to be constructed. A pneumatically actuated ball valve is used to control the flow of GOx to the combustion chamber with a check valve acting as a flow constriction helping maintain safe and steady operation during runs. Following the end of engine operation, a nitrogen purge system is activated via a solenoid valve to stop combustion and preserve the fuel grain structure for later examination. To eliminate the presence of trapped pressure in the oxygen supply system, a solenoid vent valve is used to vent GOx remaining in the supply lines following operation. To ensure steady exit condition from the chamber, static pressure is only measured in the chamber using a pressure port located in the forward bulkhead of the combustion chamber.

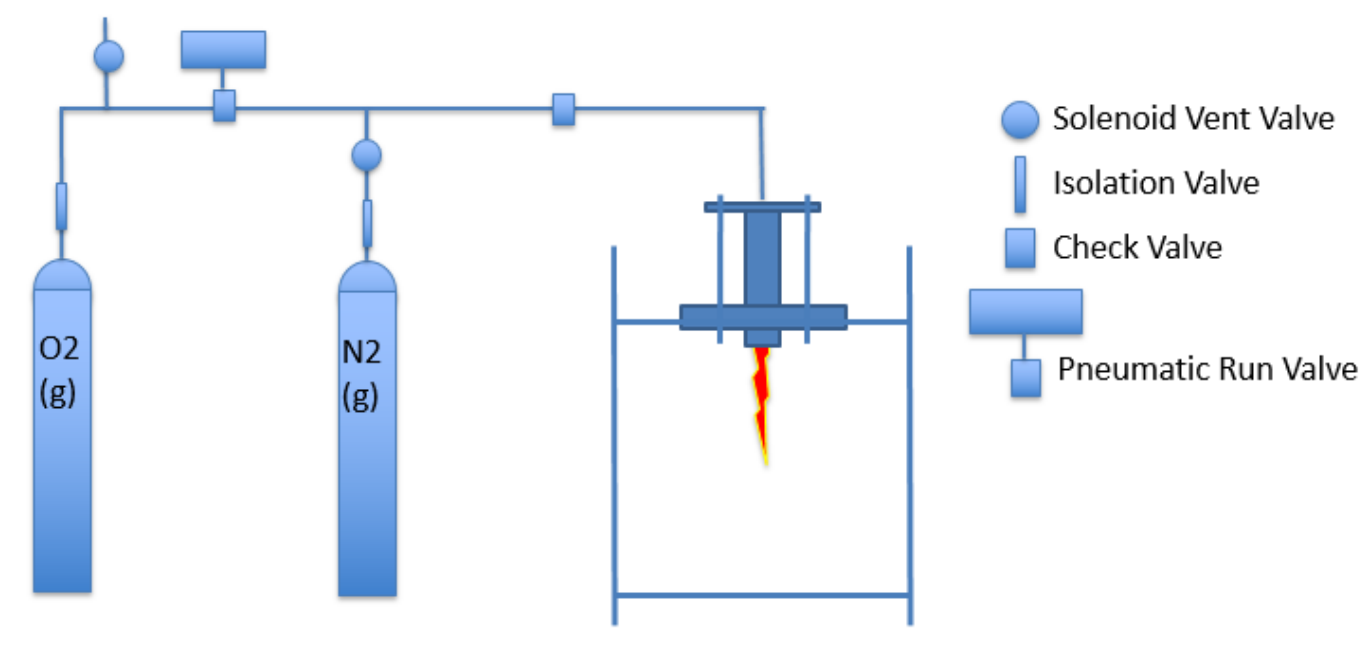

Figure 33. Hybrid rocket engine schematic diagram

The system utilizes $\mathrm{k}$ size gas bottle for all gas storage and 1/4" gas supply tubing throughout to safely supply gas flow to the combustion chamber. The oxygen supply system is set to deliver at a choked upstream pressure of 400psig, delivering 32SCFM of GOx to the combustion chamber. The chamber contains a core burning section of HTPB cast in a liner/inhibitor made of phenolic 
plastic impregnated paper, serving to contain combustion products and prevent heat transfer to the chamber walls during burn. To facilitate proper mixing in the combustion chamber, a postcombustion chamber is present at the end of the fuel section prior to the converging nozzle section. The nozzle is an off-the-shelf composite nozzle also constructed from phenolic resin impregnated paper. This ablative nozzle was chosen to allow for consistencies between runs by simple replacement of a nozzle to ensure minimal run-to-run differences due to nozzle erosion or combustion product buildup. Figure 34 below shows a cross-section view of combustion chamber under construction including the aft mixing chamber for post-combustion mixing and the off-theshelf nozzle.

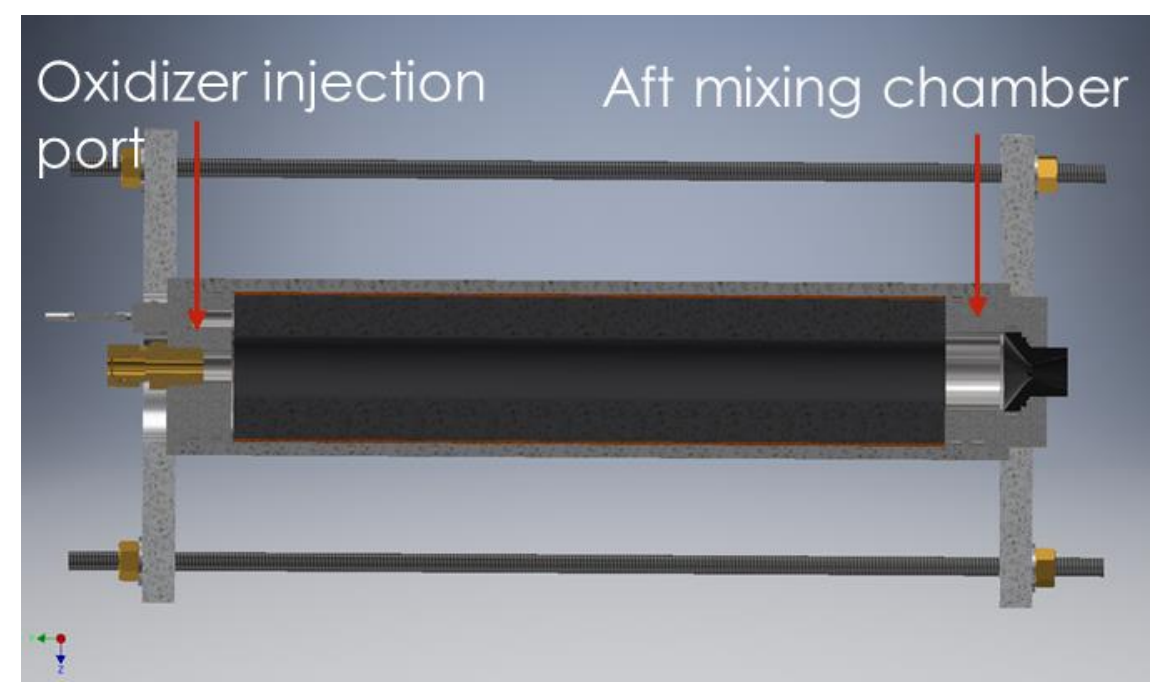

Figure 34. Hybrid rocket combustion chamber cross-section

The chamber is constructed of 6061-T6 aluminum with a nominal inner diameter of 54mm. Each end of the chamber is sealed by two internal O-rings. A single-fault-tolerant sealing surface with positive pressure is maintained to provide sealing by using tensioned threaded rods which mount to a mounting plate on either side of the chamber. 


\section{Results and Discussion}

\subsection{Dry gas phase characterization and structure}

The generation of a supersonic exhaust jet is a key component of this work. Characterization of the jet structure is important, as the overexpanded jet consists of a shock train similar to that illustrated in Figure 11 and the specific location in the shock train into which the liquid jet is injected can play a key role in the resulting spray structure. Further, the gas dynamic properties of the exhaust jet are required to evaluate parameters such as the momentum ratio and gas Weber number which allow for establishing the breakup regime of the injected liquid jet.

The air flow conditions are maintained to be identical for all the cases investigated in this work. The air flow path in the experiment is shown in Figure 22. For each test case a constant flow rate of $54 \mathrm{SCFM}$ is measured upstream of the air nozzle. An inlet static pressure of 73 psig and temperature of $67^{\circ} \mathrm{F}$ are measured by the pressure transducer and thermocouple mounted at the air inlet to the chamber as illustrated in Figure 24. These inlet conditions accelerate the air flow through the C-D nozzle section and generate an overexpanded, supersonic air jet. The Mach number at the exit plane of the nozzle is calculated to be Mach 2.5. Acceleration from the inlet conditions to this supersonic speed generates a velocity of $566 \mathrm{~m} / \mathrm{s}$, static density of $0.892 \mathrm{~kg} / \mathrm{m}^{3}$ and static temperature of $130 \mathrm{~K}$. These values are calculated using compressible flow relationships and the area ratio of the C-D nozzle. For specifying momentum ratio $q$, the conditions as expanded to ambient pressure will be used. The total pressure at the inlet to the chamber is calculated to be, $P_{o} \sim 80$ psig. This value was calculated using the measured inlet static pressure, gas mass flowrate, and the geometry of the inlet manifold to estimate the inlet total pressure. This gives an overall

pressure ratio of $\frac{P_{o}}{P_{a m b}}=6.44$. Expansion to the ambient conditions yields a flow velocity of 472.85 $\mathrm{m} / \mathrm{s}$, static density of $1.98 \mathrm{~kg} / \mathrm{m}^{\wedge} 3$, static temperature of $178.7 \mathrm{~K}$, and a Mach number of 1.76 . 
The run-to-run consistency in the supplied air pressure to the air chamber is of importance since a decrease in the total pressure upstream of the nozzle results in changes in jet structure and gas dynamic conditions. Accordingly, a threshold for total run time was set before the tank needed to be recharged. Figure 35 (left) and Figure 35 (right) below show the static pressure and temperature at the air injection manifold upstream of the air chamber over a 10 minute run time. The results here show a $\sim 5 \%$ change in the air supply static pressure after 5 minutes. This decrease in static pressure was determined to be enough of a shift from the nominal static pressure at the chamber inlet of 75 psig to necessitate recharging the outdoor air accumulation tank. The $5 \%$ decrease corresponds a decrease to $\sim 140$ psig in the accumulation tank which is nominally charged to $150 \mathrm{psig}$. The static pressure of the air entering the cylinder is relatively steady over the calibration run time.

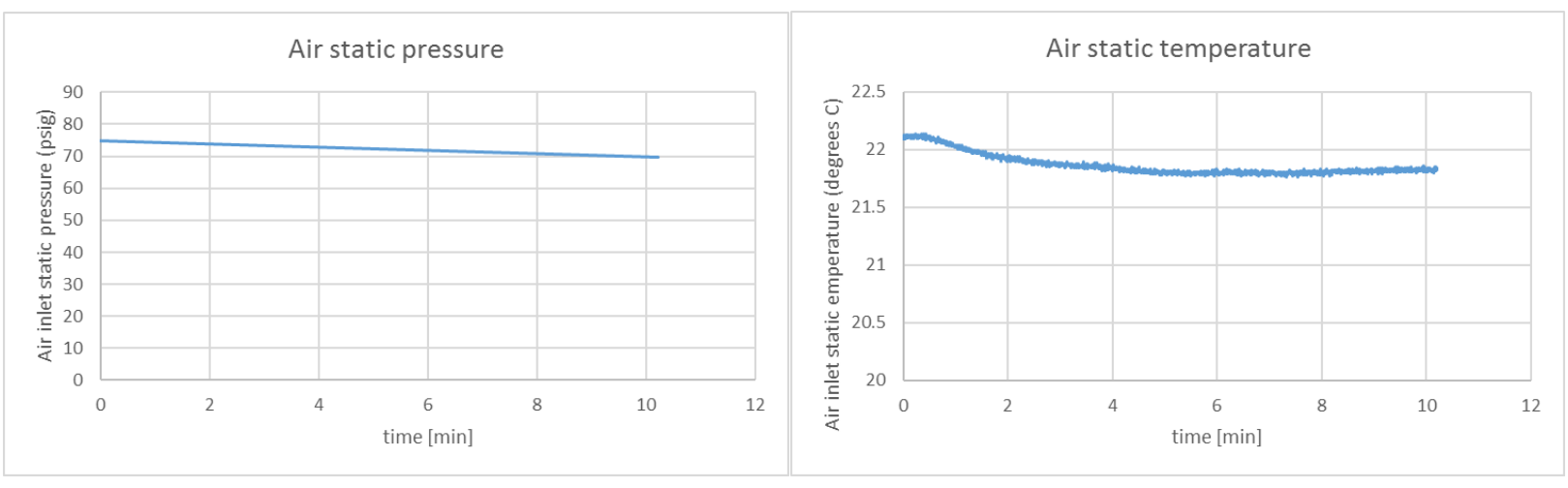

Figure 35. Long run-time compressed air supply characterization curves for static pressure (left) and temperature (right)

A supersonic plume is formed at the exit of the C-D nozzle as seen in Figure 36 (left) which shows the result as obtained from the FCS setup discussed in Section 3.3.3. The flow structure obtained from the FCS setup can be further compared with results from a compressible flow calculation performed by Danny Allgood, a collaborator on this work from NASA SSC. The CFD results are presented in Figure 36 (right). The CFD calculation used the identical geometry of the C-D nozzle with the inlet conditions specified according to experimental data. The images 
presented in Figure 36 are scaled in size so as to provide a one to one correspondence between the CFD and experimental results. Figure 36 (right) plots the density gradient magnitude contours on a linear scale starting from the nozzle throat and continuing into the ambient conditions external to the nozzle. The experimental result shown in Figure 36 (left) is a Schlieren image and as such illustrates the density gradients in the flow, allowing for good visual comparison to this sort of CFD visualization. The Schlieren image provides a good illustration of the flow structure similar to the schematic presented in Figure 11 for a supersonic overexpanded jet. As the nozzle generates an overexpanded gas flow, oblique shockwaves are present at a separation point inside the nozzle to allow the flow to recover to ambient pressure. This separation can be clearly seen in the CFD result in Figure 36 (right) and begins slightly above the exit plane of the nozzle. The separation results in the formation of a core supersonic flow surrounded by an annular subsonic flow with a recirculating region. The separation itself is a result of boundary layer separation due to an adverse pressure gradient imposed by the presence of the shock wave in the diverging section of the C-D nozzle [40]. If the overall pressure ratio $\frac{P_{o}}{P_{a m b}}$ were to be increased the shock would move downstream towards the nozzle exit and the nozzle would flow more "full".

The overexpansion of the flow is observed in the simulation result as well with the formation of oblique shocks close to the nozzle exit plane. This is followed by the shock train consisting of alternating expansion fans and oblique shock waves, which reflect off the free boundary surface, consistent with that of a supersonic overexpanded free jet. The iso-contours of Mach number plotted in Figure 37 indicate that the Mach number along the center line changes considerably as the flow proceeds downstream from the nozzle exit. Close to the exit plane, the Mach number is closer to about 2.5, which agrees well with the computed exit Mach number of 2.8 considering the early flow separation based on the total pressure in the chamber and the nozzle 
area ratio. Further downstream, the CFD results indicate that the Mach number in the core of the flow drops rapidly and the plume itself starts to disappear about 7-8 $\mathrm{cm}\left(7-8 \mathrm{~d}_{0}\right)$ downstream of the nozzle exit. Some parametric studies were conducted in the CFD code to understand the influence of chamber pressure and temperature on the jet structure. While the inlet pressure was found to have a strong influence on the jet structure, temperature within \pm 5 degrees was found to have a negligible influence.

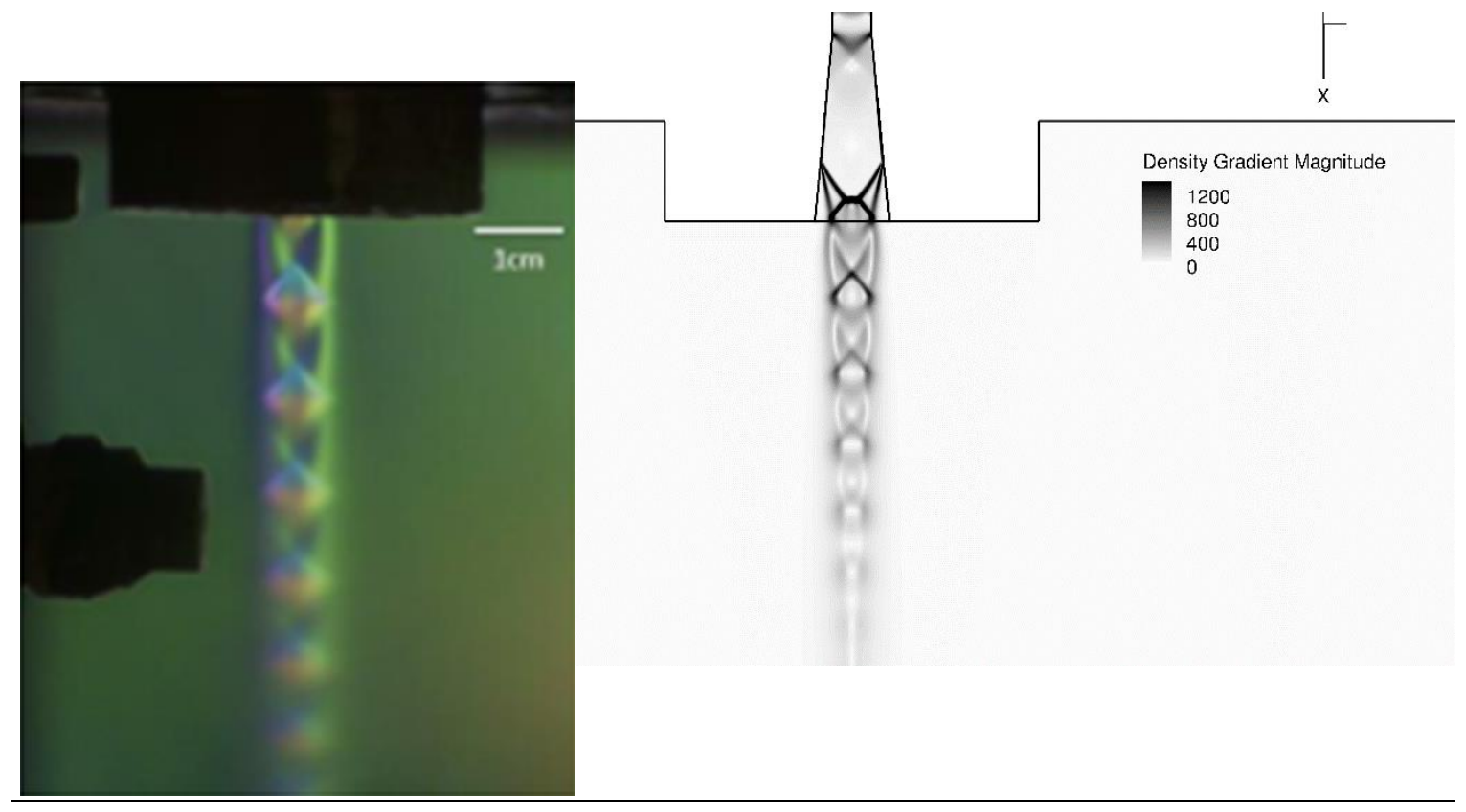

Figure 36. Left: Gas phase imaged using FCS, Right: Gas phase simulation results plotting density gradient magnitude (Source: Danny Allgood, NASA SSC) 


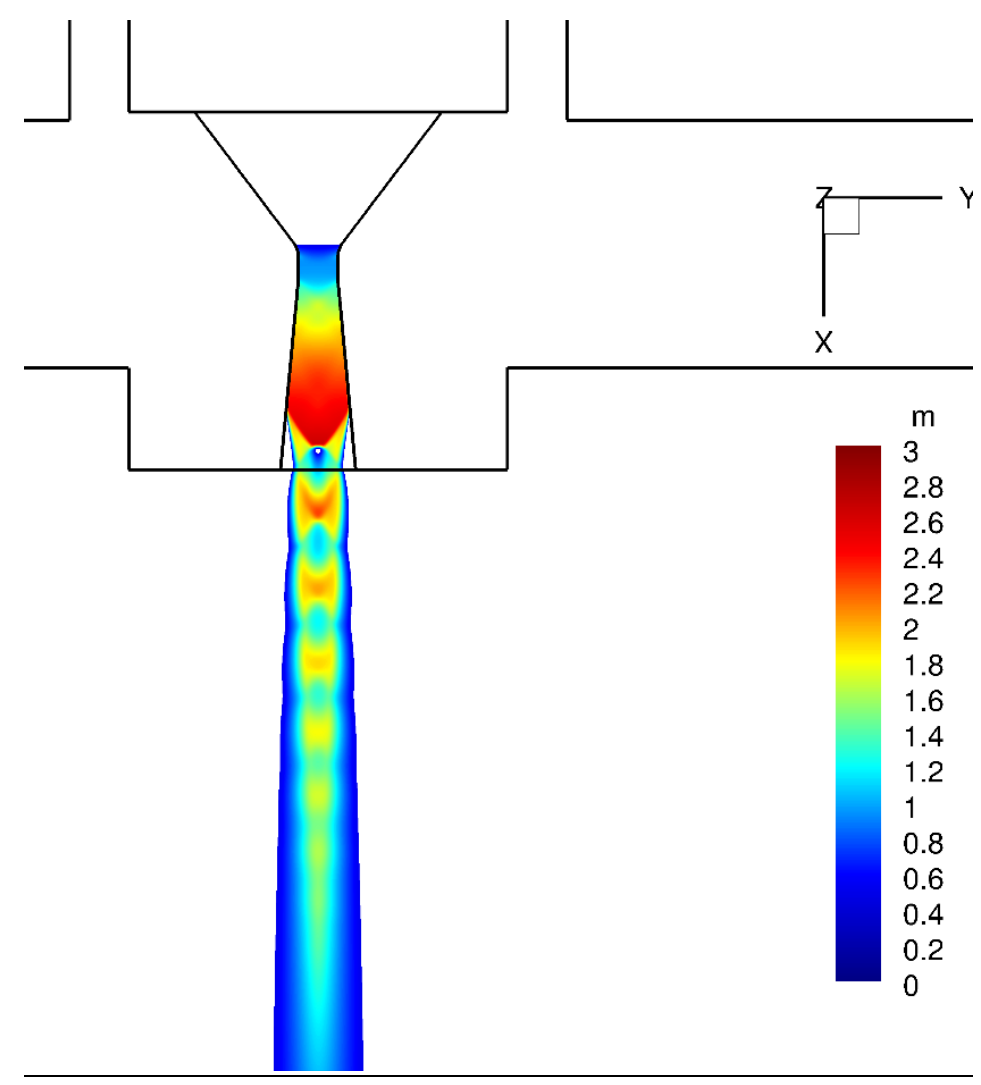

Figure 37. Gas phase simulation results plotting Mach number iso-contours (Source: Danny Allgood, NASA SSC)

A DSLR camera is used to acquire images in the FCS setup and is operated with a frame rate of 60 frames/second. The resulting image observed in Figure 36 (left) is time averaged over a period of about $17 \mathrm{~ms}$. A characteristic flow time scale can be calculated using the jet exit velocity and the nozzle exit diameter as shown in equation 4.1 below.

$$
t_{\text {flow }}=\frac{d_{\text {jet }}}{u_{\text {jet }}}=\frac{0.00965 \mathrm{~m}}{560 \frac{\mathrm{m}}{\mathrm{s}}}=17 \mu \mathrm{s}
$$

This indicates that the image acquired using the FCS setup is averaged roughly over 1000 flow time scales. Despite the averaging, the shock structures observed in the image are quite sharp indicating that the exhaust plume is steady and past an initial start-up transient, moves very little in space. The spacing of shockwave cells within each flow is a good indicator of proper agreement between the experimental and CFD results, this comes to be $\sim 9 \mathrm{~mm}$ for both the computational and experimental results. 


\subsection{Gas phase behavior with liquid injection}

After having characterized the supersonic air jet in isolation, the change in its structure due to interaction with a liquid jet injected in a normal direction is investigated. As mentioned earlier, the air flow conditions are maintained constant for all test cases with a total pressure 80psig at the inlet. Air pressure exerted upstream in the reservoir shown in Figure 20 is varied between 22-112 psig to supply the necessary back pressure at the nozzle. Water injection static pressure measured at the nozzle as shown in Figure 24 ranges between 20-100psig after losses between the reservoir and injector. Water flow rate is measured using the in-line flowmeter. In all cases investigated in this work, water is injected in a direction normal to the axis of the air exhaust plume.
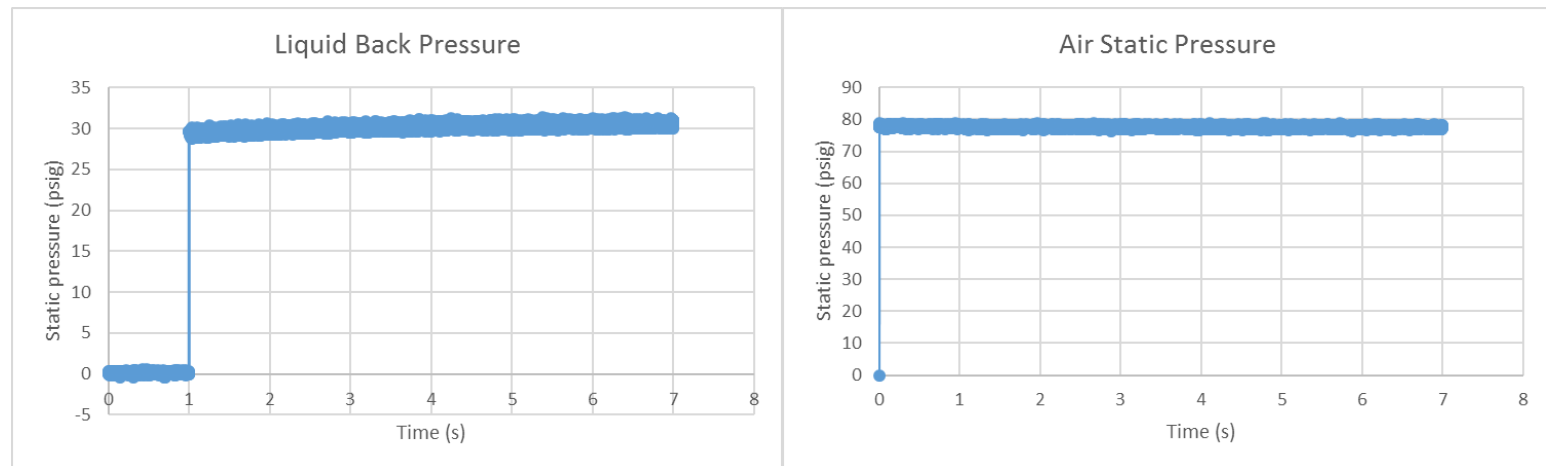

Figure 38. Left: Liquid back pressure for $q=0.937$, Right: Air inlet static pressure for $q=0.397$
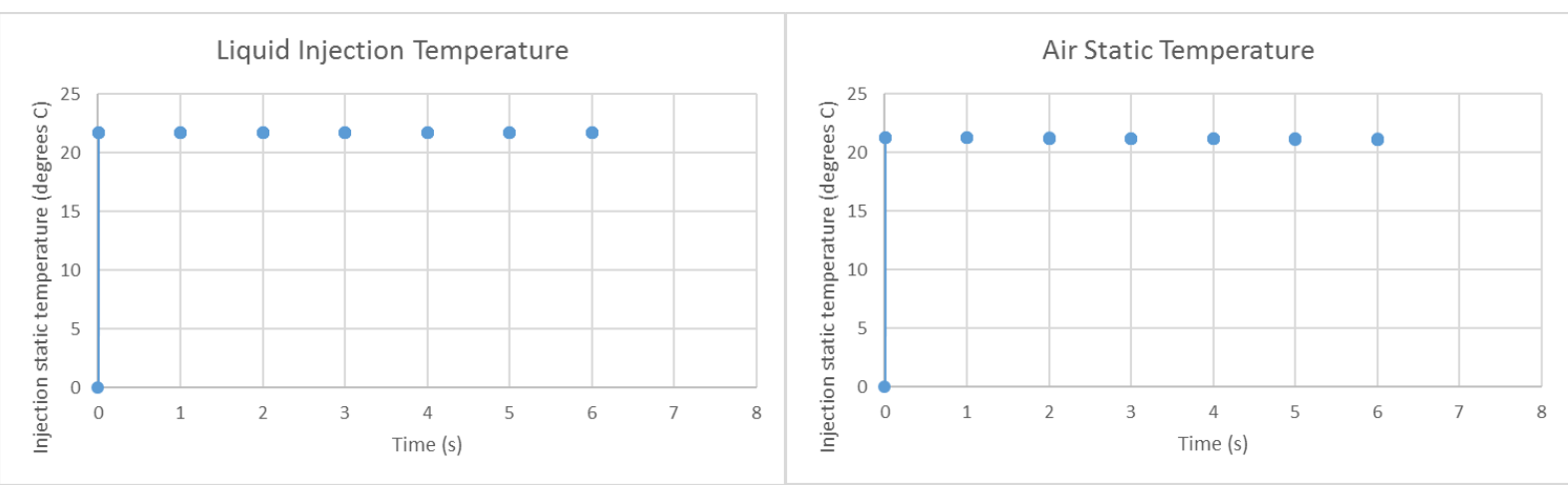

Figure 39. Left: Liquid static temperature for $q=0.937$, Right: Air inlet static temperature for $q=0.937$ 
Several dimensionless groups can be calculated to characterize the experimental conditions. This includes the gas Weber $\left(W_{G}\right)$ number, the Ohnesorge $(\mathrm{Oh})$ number, momentum ratio $(q)$, and the liquid jet Reynolds number based on diameter $\left(R e_{D}\right)$. While the gas Weber number and momentum ratios have been defined earlier, the Ohnesorge number is given by,

$$
O h=\frac{\mu_{l}}{\left(\rho_{l} d_{0} \sigma_{l}\right)^{1 / 2}}
$$

Where $\mu_{l}, \rho_{l}$, and $\sigma_{l}$ are the liquid dynamic viscosity, density, and surface tensions respectively and $d_{0}$ is the water jet diameter. The Ohnesorge number compares viscous forces to inertial and surface tension forces in the liquid. The liquid jet Reynolds number is given by,

$$
R e_{D}=\frac{\rho_{l} d_{0} V_{j e t}}{\mu_{l}}
$$

Where $V_{j e t}$ is the water jet exit velocity.

For the conditions investigated in this work, three nozzles have been examined and will be referred to as nozzle $1(.06 "(1.52 \mathrm{~mm})$ diameter), nozzle $2(.04 "(1.02 \mathrm{~mm})$ diameter), and nozzle $3(.03 "(0.76 \mathrm{~mm})$ diameter $)$. For each of the liquid injection cases examined in this work, tables are provided below listing the nozzle diameter, Ohnesorge number, and gas Weber number. Parameters corresponding to each test condition are also summarized in each table. Table 2 below shows the operating conditions for nozzle 1 . Total flowrate and velocities measured through the nozzle orifice for the range of injection pressures are examined. The measured velocity corresponds to an estimate based on the measured volume flow rate, liquid density, and nozzle exit flow area. The measured velocities were compared with a calculated velocity obtained using a traditional incompressible orifice flow calculation given by Equation 4.2 below. Note that a discharge coefficient is required to calculate the flow velocity based on Equation 4.2. By 
comparing the measured velocity with the calculated value, a discharge coefficient was estimated. For the remaining nozzles, the same discharge coefficient was applied along with Equation 4.2 to determine the flow velocity given that all nozzles are of the same design and the only difference is in the size of the orifice opening.

$$
v=C_{d} \sqrt{\frac{2 \Delta P}{\rho}}
$$

Given the small error of $<4 \%$ when comparing the calculated and measured exit velocities this discharge coefficient was used in the calculations for the smaller nozzles given the high turbulent Reynolds numbers for all injection cases.

Table 2. Nozzle characterization for nozzle 1

\begin{tabular}{|c|c|c|c|c|c|c|c|c|c|}
\hline $\begin{array}{l}\text { Nozzle 1 } \\
\mathrm{D}=.06 "\end{array}$ & $\mathrm{We}=6138$ & $\mathrm{Oh}=.0032$ & & & & & & & \\
\hline $\begin{array}{l}\text { Injection } \\
\text { Pressure } \\
\text { [psig] }\end{array}$ & 20 & 30 & 40 & 50 & 60 & 70 & 80 & 90 & 100 \\
\hline $\begin{array}{l}\text { Measured } \\
\text { Flowrate } \\
{[\text { GPM] }}\end{array}$ & 0.4753 & 0.593 & 0.693 & 0.791 & 0.865 & 0.918 & 1.00 & 1.05 & 1.12 \\
\hline $\begin{array}{l}\text { Measured } \\
\text { Velocity } \\
{[\mathrm{m} / \mathrm{s}]}\end{array}$ & 16.44 & 20.51 & 23.97 & 27.35 & 29.91 & 31.75 & 34.58 & 36.31 & 38.73 \\
\hline $\begin{array}{l}\text { Calculated } \\
\text { Velocity } \\
{[\mathrm{m} / \mathrm{s}]}\end{array}$ & 16.63 & 20.37 & 23.52 & 26.30 & 28.81 & 31.12 & 33.26 & 35.28 & 37.19 \\
\hline$\%$ Error & 1.18 & -0.67 & -1.86 & -3.87 & -3.70 & -1.99 & -3.82 & -2.84 & -3.99 \\
\hline$q$ & 0.624 & 0.937 & 1.249 & 1.561 & 1.873 & 2.185 & 2.497 & 2.810 & 3.122 \\
\hline$R e_{D}$ & 24064 & 29472 & 34032 & 38049 & 41680 & 45020 & 48128 & 51048 & 53810 \\
\hline
\end{tabular}

As flow velocity across each orifice is independent of the cross sectional area, the momentum ratio for each nozzle case does not change, though the Reynolds number and volume 
flow rate change. Table 3 and Table 4 below show the cases explored for the smaller nozzles nozzle 2 and nozzle 3 respectively.

Table 3. Nozzle 2 injection parameters

\begin{tabular}{|c|c|c|c|c|c|c|c|c|c|}
\hline $\begin{array}{l}\text { Nozzle } 2 \\
\mathrm{D}=.04 "\end{array}$ & $\mathrm{We}=4092$ & $\mathrm{Oh}=.0039$ & & & & & & & \\
\hline $\begin{array}{l}\text { Injection } \\
\text { Pressure } \\
\text { [psig] }\end{array}$ & 20 & 30 & 40 & 50 & 60 & 70 & 80 & 90 & 100 \\
\hline $\begin{array}{l}\text { Calculated } \\
\text { Velocity } \\
{[\mathrm{m} / \mathrm{s}]}\end{array}$ & 16.63 & 20.37 & 23.52 & 26.30 & 28.81 & 31.12 & 33.26 & 35.28 & 37.19 \\
\hline$q$ & 0.624 & 0.937 & 1.249 & 1.561 & 1.873 & 2.185 & 2.497 & 2.810 & 3.122 \\
\hline$R e_{D}$ & 16043 & 19649 & 22688 & 25366 & 27787 & 30014 & 32086 & 34032 & 35873 \\
\hline
\end{tabular}

Table 4. Nozzle 3 injection parameters

\begin{tabular}{|c|c|c|c|c|c|c|c|c|c|}
\hline $\begin{array}{l}\text { Nozzle } 3 \\
\mathrm{D}=.03 "\end{array}$ & $\mathrm{We}=3067$ & $\mathrm{Oh}=.0045$ & & & & & & & \\
\hline $\begin{array}{l}\text { Injection } \\
\text { Pressure } \\
\text { [psig] }\end{array}$ & 20 & 30 & 40 & 50 & 60 & 70 & 80 & 90 & 100 \\
\hline $\begin{array}{l}\text { Calculated } \\
\text { Velocity } \\
{[\mathrm{m} / \mathrm{s}]}\end{array}$ & 16.63 & 20.37 & 23.52 & 26.30 & 28.81 & 31.12 & 33.26 & 35.28 & 37.19 \\
\hline $\mathrm{q}$ & 0.624 & 0.937 & 1.249 & 1.561 & 1.873 & 2.185 & 2.497 & 2.810 & 3.122 \\
\hline$R e_{D}$ & 12032 & 14736 & 17016 & 19025 & 20840 & 22510 & 26064 & 25524 & 26904 \\
\hline
\end{tabular}

We now begin to examine the effect of liquid injection on the shockwave structure in the gas phase. The resulting behavior will be examined as a function of water jet injection location and jet momentum ratio for nozzle 1 . Figure 40 below shows the change induced in the shockwave structure by varying the water injection locations for a fixed momentum ratio of $\mathrm{q}=1.873$. The bottom row of images in Figure 40 are zoomed in versions of the images in the top row for closer inspection. The three images in Figure 40 roughly correspond to water injection: between the $2^{\text {nd }}$ 
and $3^{\text {rd }}$ Mach diamonds in the shock train, almost directly into the oblique shock waves for the 3rd Mach diamond in the shock train, and just below the oblique shocks of the $3^{\text {rd }}$ Mach diamond in the shock train. In all three cases, the images show the presence of a strong bow shock at the liquid gas interface. However, the separation shock present in traditional jet in crossflow as seen in Figure 5(left) is not observed. Note that the leading edge shock seen in Figure 5(left) is caused by the leading edge of the flat plate used in that experiment and should not be expected in this work. The presence of stationary Mach diamonds in this overexpanded gas flow yields some further interesting characteristics not evident in traditional confined supersonic jet in crossflow experiments. For the middle image in Figure 40, the bow shock directly interacts and appears to merge with the forward oblique shock waves for the $3^{\text {rd }}$ Mach diamond. For the right image in Figure 40, a flattening of the forward oblique shockwaves occurs as they are forced to follow the contour of the bow shock produced upstream of the water jet. The bow shock itself, which is formed near the liquid boundary after the liquid jet injection point enters the expansion region rear of the Mach diamonds is weakened due to the presence of the upstream shock waves. This general behavior is exhibited for water injection near each successive Mach diamond with further downstream interactions resulting in weaker shockwaves as total pressure present in the gas flow decreases due to viscous dissipation. Globally, the penetration distance of the liquid phase appears not to be affected by the location of the liquid injection point indicating that the penetration rather is a strong function of the jet momentum ratio. One other characteristic feature observed is the widening of the shock train structure upstream of the jet/spray boundary. The blockage of the flow path by the water jet/spray results in this widening effect. 


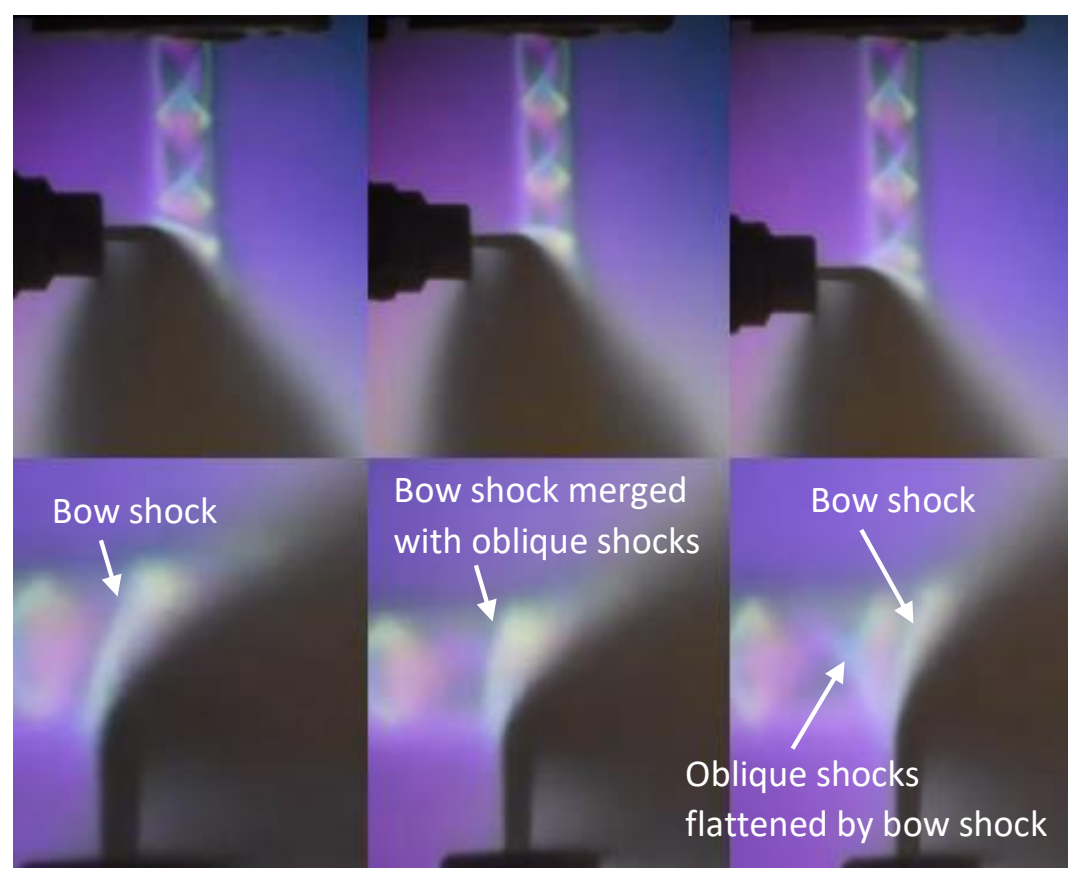

Figure 40. Shock structure behavior at varied injection locations

Next, we examine the shockwave structure for a low momentum liquid injection case. Figure 41 below shows an image obtained from the FCS setup for a water injection pressure of about $15 p s i g$ and a jet momentum ratio of $q=0.468$. The changes in the shock structure due to the water injection process are well captured by the FCS imaging. In this case, the water jet does not completely penetrate the air jet as is the case in the images shown in Figure 40. A strong, stationary bow shock is formed upstream of the injection location. A weak secondary shock, which is harder to observe is also formed very close to the leading edge of the injection point. Parts of the Mach diamonds downstream of the injection location are still visible in the images shown in Figure 9, but are considerably blurred presumably due to the spray mist of water formed in the field of view. The strong bow shock is a distinctive feature which is observed for all the cases studied for higher momentum ratio cases. The weak secondary shock is more obviously seen for the lower water injection pressure cases and is harder to detect at higher injection pressures. Figure 41 also appears to show the shock train being deviated slightly away from the injection plane past the injection 
location. No changes are observed in the shock train upstream of the injection location consistent with the supersonic nature of the flow. A significant difference between the current test configuration and the well-studied jet-in-crossflow is that there is no solid boundary constraining the air or liquid flows. This allows the injected water jet more flexibility in the path it can take once it impinges on the air jet. It is highly likely also that part of the liquid jet might try to move around the periphery of the air jet without penetrating into it. This is not currently observable given our present diagnostics but is of interest to pursue in future experiments. Finally, the image in Figure 41 shows a fine spray being formed upon impingement of the water jet on the air jet. The structure of the spray will be described in the next section.

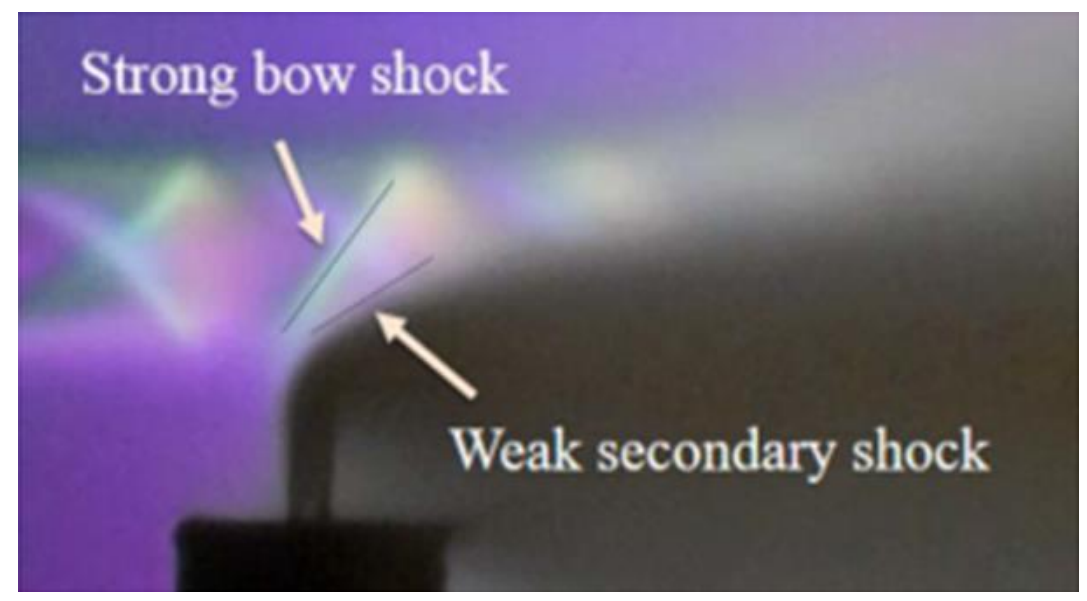

Figure 41. FCS of low momentum ratio liquid injection

\subsection{Spray penetration behavior and morphology}

\subsubsection{Liquid primary breakup process}

To begin examining the structure of the spray, the morphology of the initial spray breakup will be examined as it is of strong influence on the global behavior of the flow and on the spray particle size and velocity downstream. Figure 42 below shows a version of the regime diagram introduced in Figure 13 which has been extended to include the range of Weber numbers of interest in the current work and the locations of the current test cases for nozzles 1, 2 and 3 have been 
marked. Due to the location of the test cases of interest within this regime map we expect to see a combination of shear breakup and surface breakup phenomena with increasing momentum ratio. This behavior is explored for nozzle 1 using volume illuminated high-speed photography. The images for these test cases were taken at 20,000 fps using the same lighting orientation shown in Figure 29.

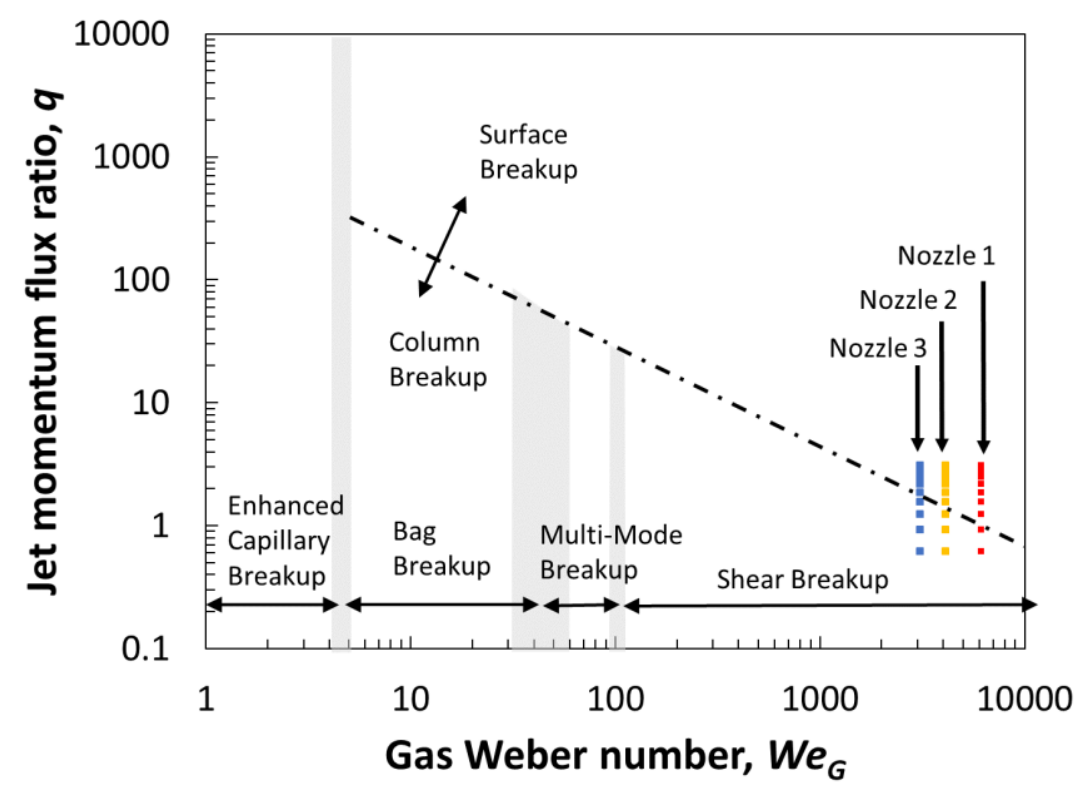

Figure 42. Modified regime diagram showing cases of interest

Figure 43 and Figure 44 below show still images taken from the high-speed video for nozzle 1 at momentum ratios $\mathrm{q}=0.624$ and $\mathrm{q}=2.497$ respectively. The behavior observed in the jet breakup process in Figure 43 is consistent with a shear breakup while the process observed in Figure 44 is more consistent with a surface stripping type breakup. The shear breakup process is characterized by shearing of liquid packets from the coherent jet without wave propagation along the jet surface and subsequent secondary breakup of these distinct liquid packets as shown by increased pixel intensity in the diffused liquid phase downstream. The surface stripping seen in Figure 44 is characterized by a higher level of initial liquid breakup as liquid is stripped in layers 
from the coherent jet surface, resulting in an initially smaller particle size near the gas/liquid interface and the extension of liquid ligaments not present at the lower momentum ratio case. The time difference between each frame shown below $\Delta t=.1 \mathrm{~ms}$.

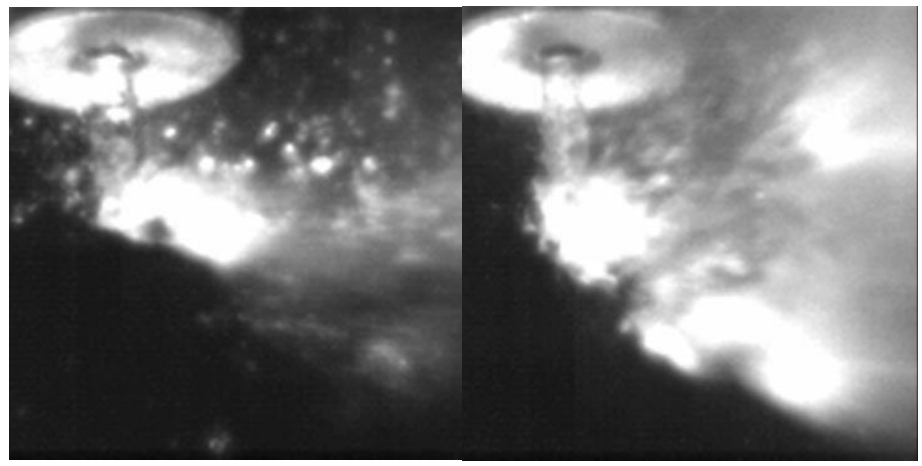

Figure 43. High-speed video still images showing shear breakup regime, nozzle $1 q=0.624$
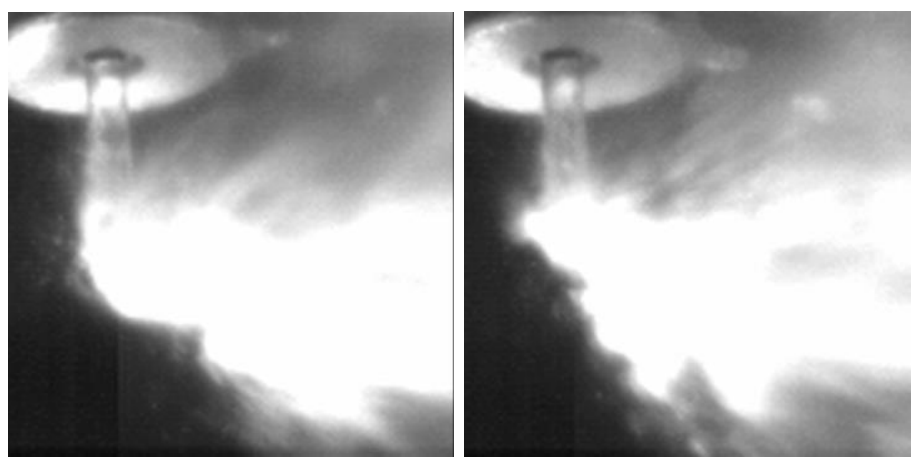

Figure 44. High-speed video still images showing surface stripping breakup regime nozzle $1 q=2.497$

To better examine the dynamic behavior of the primary jet breakup process we attempt to use the method of principle orthogonal decomposition (POD) discussed in Section 3.4. Figure 45 below shows a snapshot in time, the average of all snapshots and a selection of several of the most energetic orthogonal modes for nozzle 1 at $\mathrm{q}=0.624$, and Figure 46 shows the same for an increased momentum ratio of $\mathrm{q}=3.122$. The behavior in each case agrees qualitatively with the results presented in [38]. The more energetic lower order modes show a contrast in the scale of the turbulent structures, with an increase in the structure size at increased momentum ratio, showing 
the increased ability of the liquid jet to resist the influence of the gas jet; leading to the transition from gas dominated shear breakup to liquid dominated surface primary breakup modes.
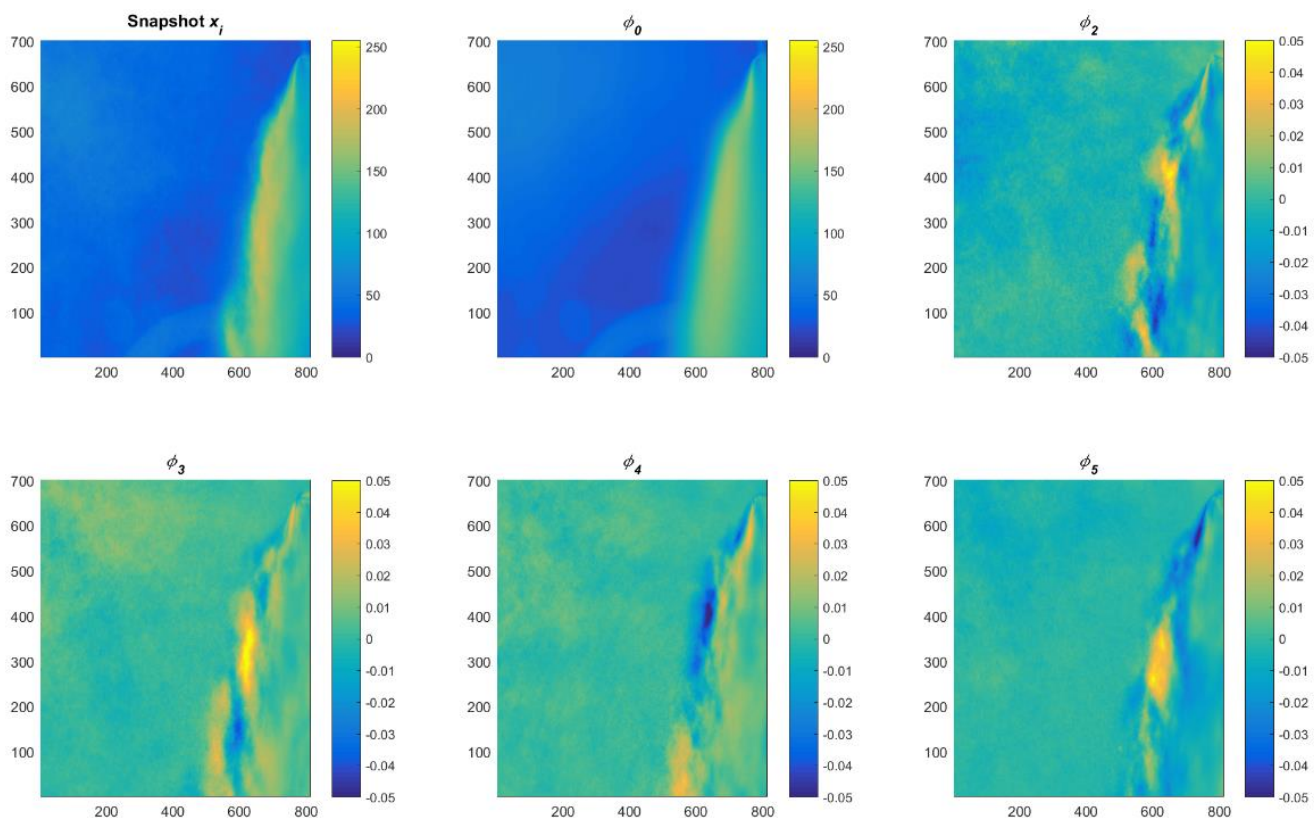

Figure 45. Snapshot, and 0th, 2nd, 3rd, 4th, and 5th orthogonal modes for $q=0.624$
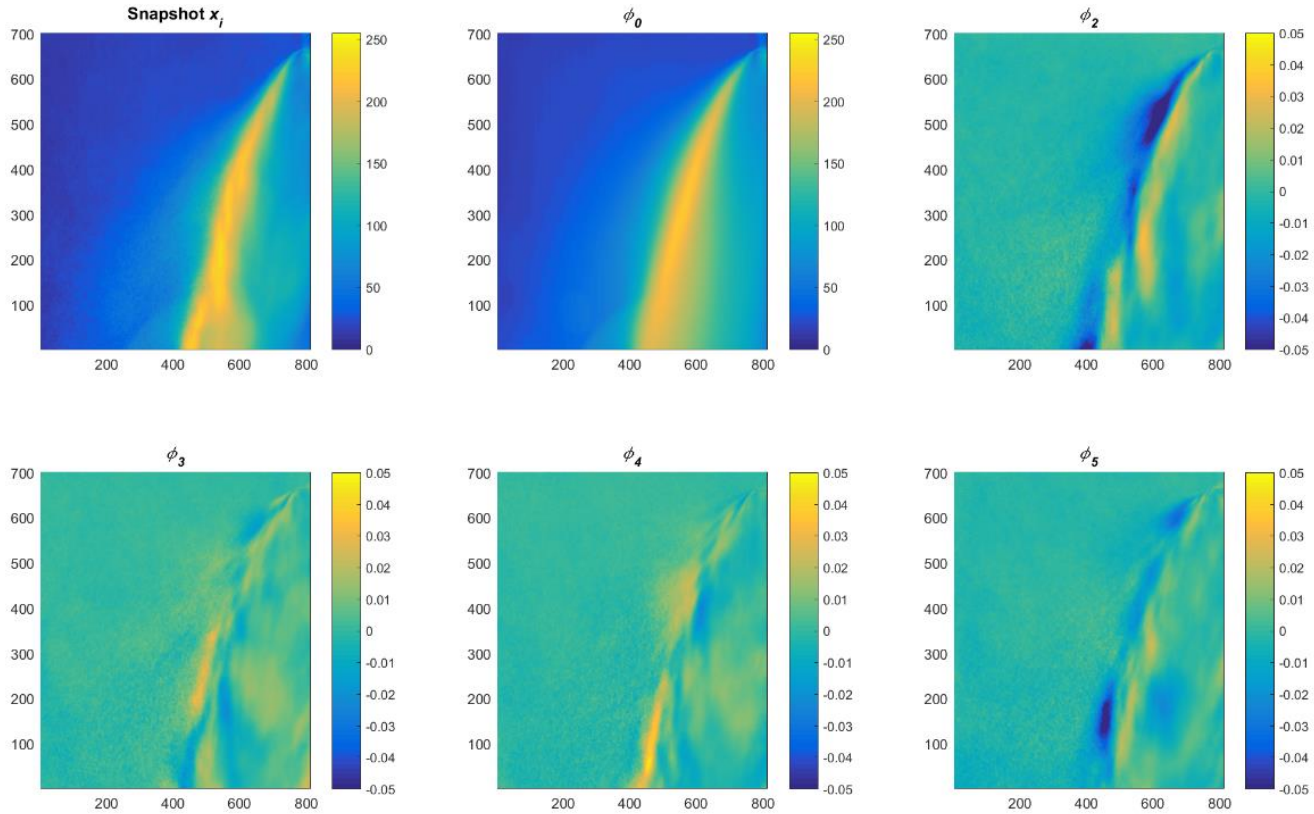

Figure 46. Snapshot, and 0th, 2nd, 3rd, 4th, and 5th orthogonal modes for $q=0.624$ 


\subsubsection{Liquid penetration distance}

The liquid penetration into the gas jet is of importance since it signifies the ability of the liquid jet to cool the supersonic air jet in applications relevant to NASA SSC. While jet penetration has been exhaustively investigated for the traditional jet-in-crossflow configuration, very little information is available for the penetration of a free liquid jet into a supersonic air jet. The liquid jet penetration distance in the global flow interactions of the two jets of interest as well as in the confined region local to the gas flow potential core will now be presented and discussed.

In order to investigate jet penetration, experiments were carried out for the various test cases mentioned in Table 2, Table 3, and Table 4. For each test case, high speed videos were obtained of the jet interaction at a frame rate of 20,000 fps. The images were then processed individually in MATLAB as illustrated in Figure 47. For each high-speed image obtained over an exposure time of $0.5 \mathrm{~ms}$, a smoothed image is first obtained using a Savitzky-Golay filter, a filter which fits a low order polynomial to the 2D pixel intensity data using the method of least squares to decrease noise in each frame. This smoothed image is then transformed to a binary image using a consistent threshold value chosen independently for each nozzle data set. The choice of this threshold value sets the level of pixel intensity chosen to represent the spray edge. Any small objects outside of the larger spray image generated by reflections or holes in the image are removed and the contour of this is overlaid on the original snapshot, with the furthest edge from the injection point representing the spray penetration edge. Figure 47 below shows this process for a single snapshot. 

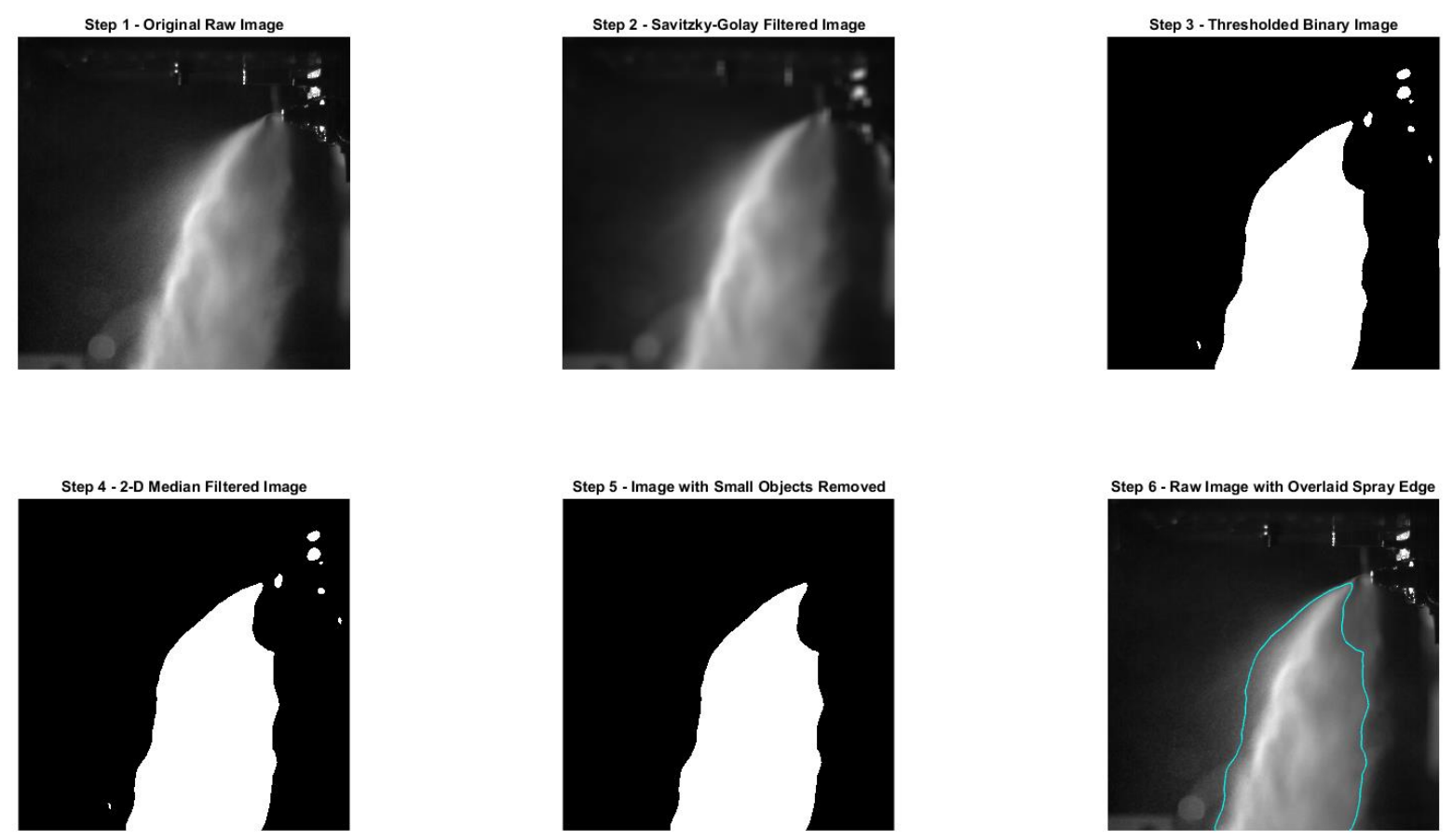

Figure 47. Spray edge detection process

To properly detect the average location of the spray boundary as the boundary changes with time, many of these identifications of the spray edge for consecutive snapshots must be averaged for each set of experimental parameters. Depending on the nature of the flow the random fluctuations seen in the edge location can be large or small as shown in Figure 48. Here the edge location for each snapshot is traced in light blue, and the ensemble average edge location is traced in dark blue. Additionally, the gas jet centerline and projected nozzle exit edges are traced in red and light green respectively. These edge locations are traced over an averaged image and are results for 100 snapshots. Here, the $(0 \mathrm{~mm}, 0 \mathrm{~mm})$ origin location corresponds to the bottom right edge of the air nozzle, this location will be used as a physical reference for the spatial location of the spray edge in the analysis to follow. 

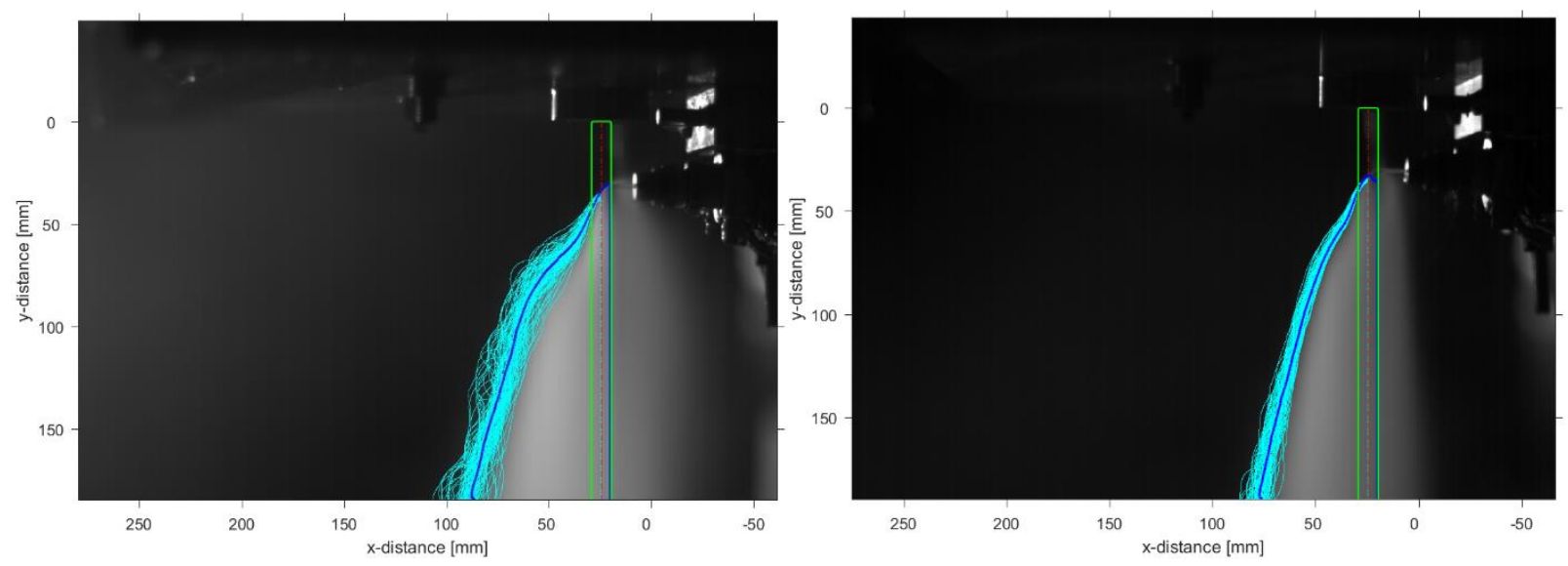

Figure 48. Left: Edge location tracking and average for Nozzle $1 q=0.62437$, Right: Edge location tracking and average for Nozzle 3 q=2.497

Using this located spray boundary location, we can now proceed to assess the penetration of the spray both in the gas phase and globally, as well as examine some of the general characteristics of each case. Figure 49 below shows averaged pixel intensity values for nozzles 1 , 2, and 3 for momentum ratios of $\mathrm{q}=0.624, \mathrm{q}=1.56$, and $\mathrm{q}=3.12$ averaged over 1000 snapshots. For increasing momentum ratio and increasing orifice size an increased global penetration distance is seen as expected. The largest penetration distance is evident in the top right image and smallest penetration distance is shown in bottom left image. For the larger diameter water nozzle cases the spray quickly penetrates fully through the gas jet exiting into the ambient where it is no longer influenced by the gas phase, even for a low momentum ratio, indicating a strong dependence for global spray penetration on the diameter ratio of the liquid and gas phase nozzle exit areas. Again, the projected edges of the gas nozzle are shown in light green, the gas flow centerline in red, and the computed average edge location in blue. 


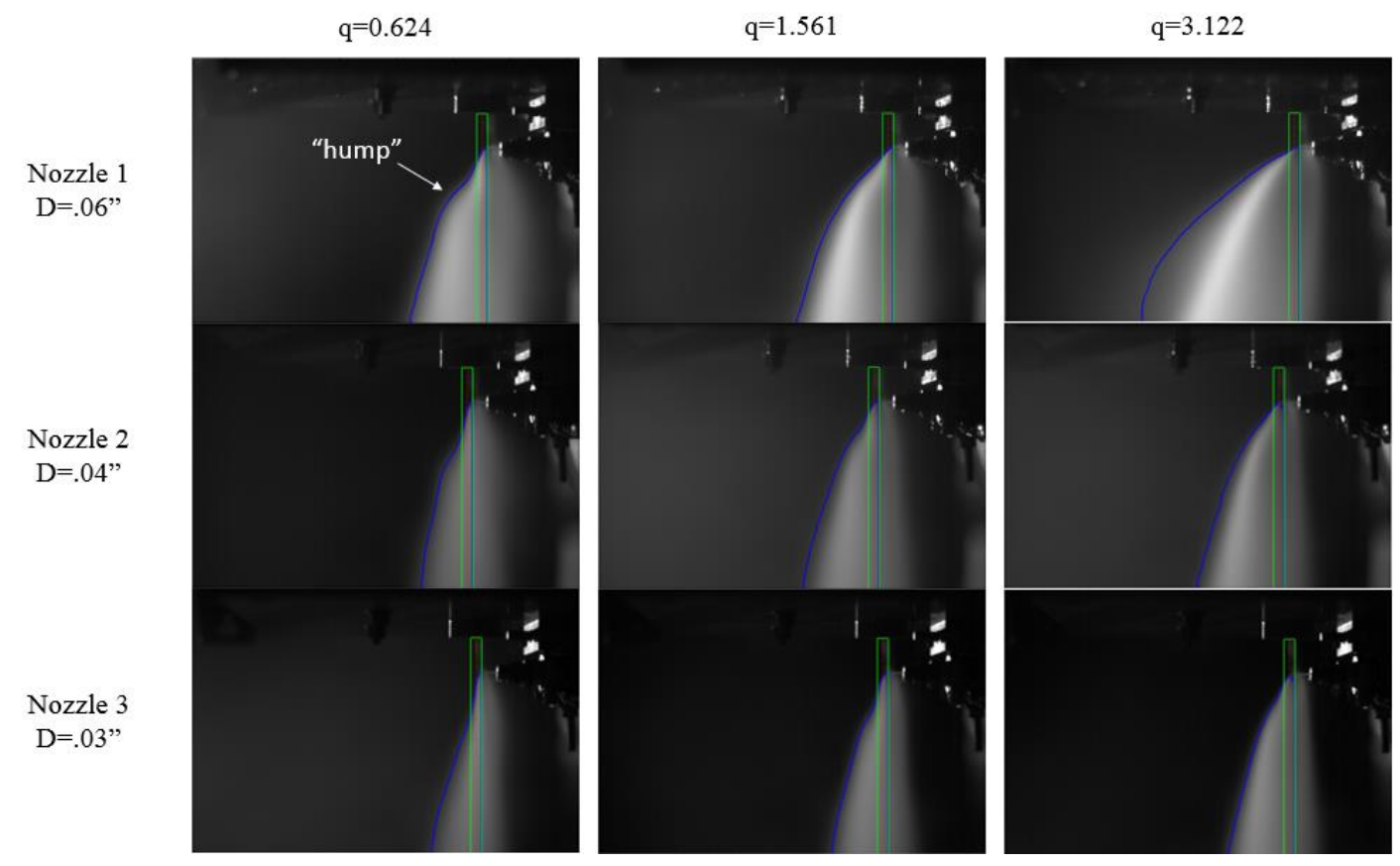

Figure 49. Global penetration data for all nozzles at selected momentum ratio

Another physical feature present in the averaged intensity plots shown in Figure 49 above is the presence of a "hump" in several of the cases, especially for smaller diameter and smaller momentum cases. The "hump" is observed at a distance downstream of the injection point where the contour of the spray edge experiences a sudden change in curvature. Physically this could represent a sudden change in particle size in the dispersed liquid phase, resulting in a sudden decrease in density and expansion. This edge feature was also observed in preliminary two-phase CFD results (conducted by NASA SSC collaborators). This feature will be examined in detail in future work using PDPA to examine the droplet sizes at this downstream location. The current PDPA data obtained has been taken at a distance too far downstream to capture this phenomenon.

Next we will examine the penetration distances obtained for each nozzle at various injection pressures. Both, the global penetration as well as the penetration solely within the projected air jet column envelope will be investigated. The jet penetration correlations developed for the traditional jet-in-crossflow configuration are expected to apply only within the air jet 
column envelope, where the water jet is actually moving through a supersonic crossflow. Figure 50 and Figure 51 show the global and gas jet penetration data for nozzle 1; Figure 52 and Figure 53 show global and gas jet penetration data for nozzle 2; and Figure 54 and Figure 55 show global and gas jet penetration data for nozzle 3, respectively. The downstream location and penetration height are normalized by the liquid jet diameter for each case, each figure also indicates the location of the gas jet projected edge location as a reference to locate the contact surface of the two jets.

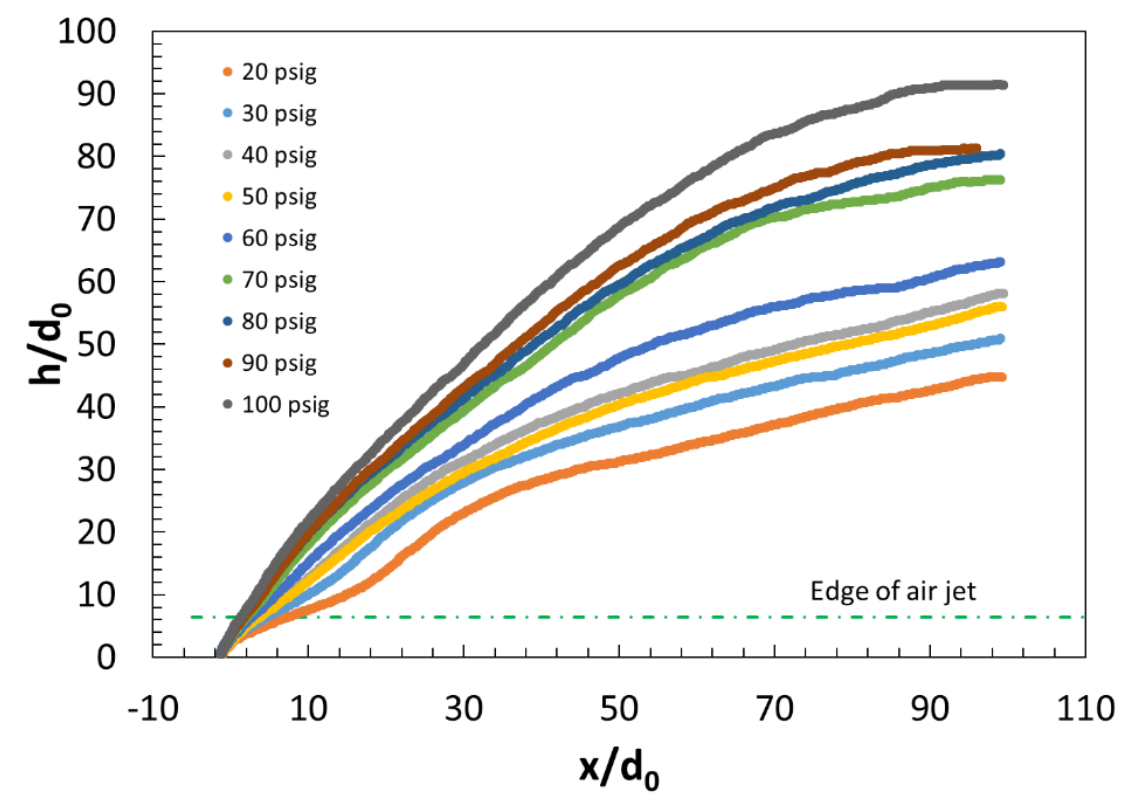

Figure 50. Nozzle 1 global penetration 


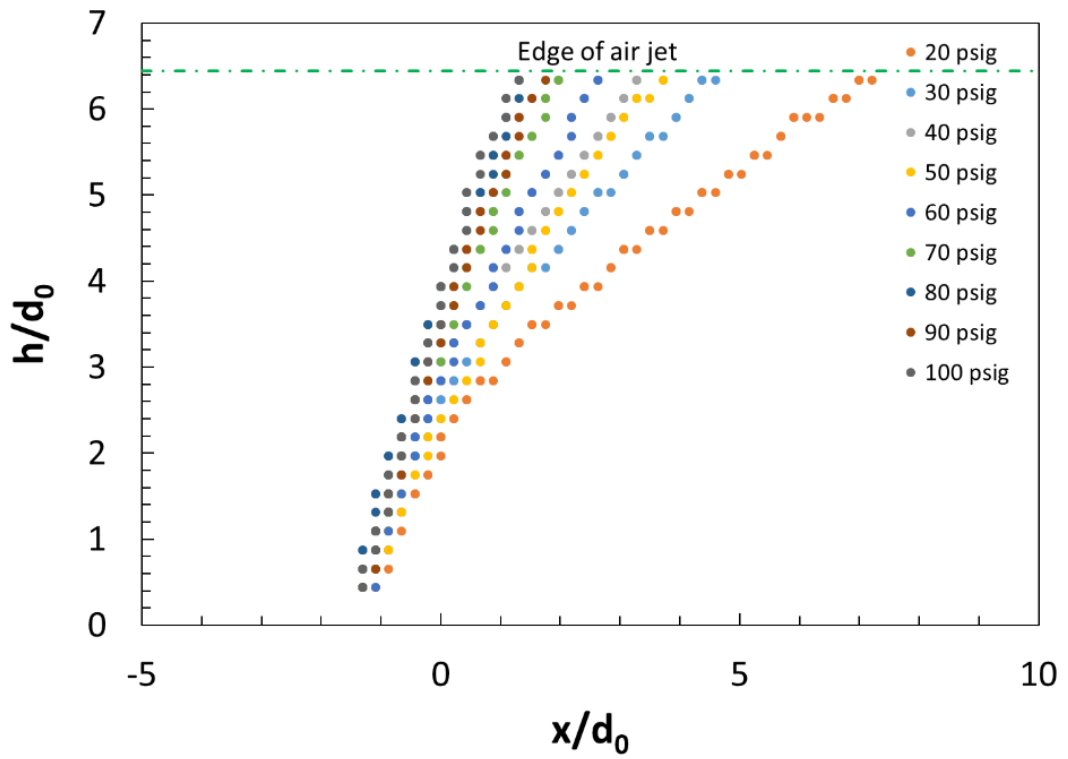

Figure 51. Nozzle 1 air jet penetration

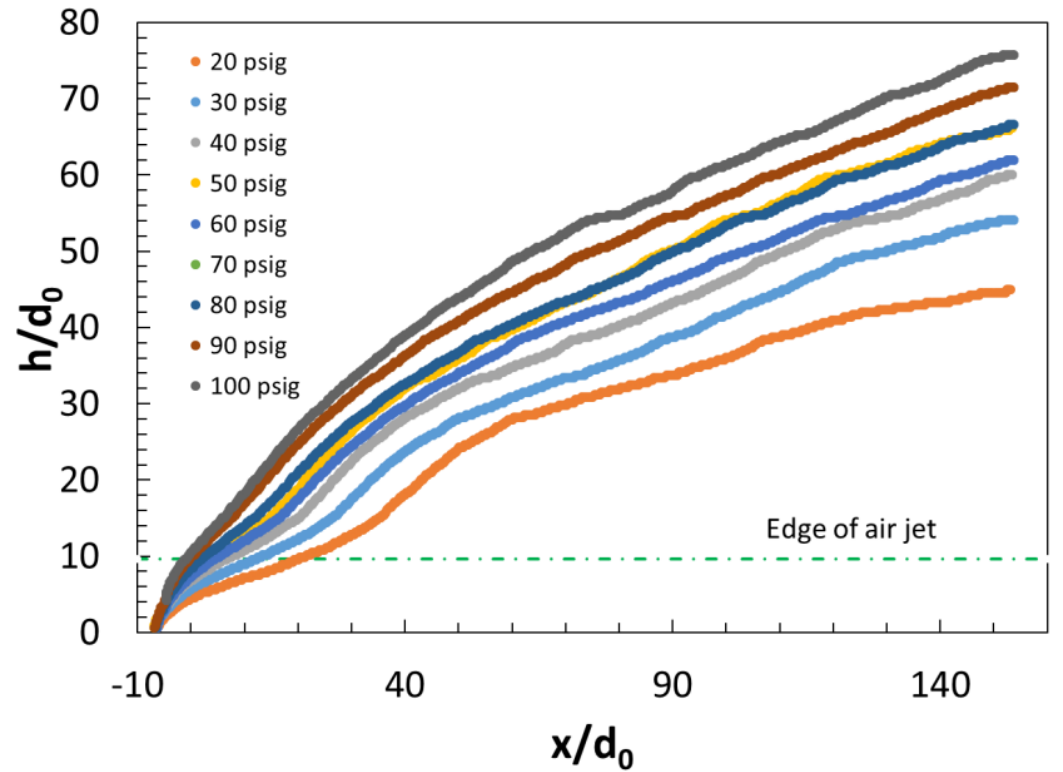

Figure 52. Nozzle 2 global penetration 


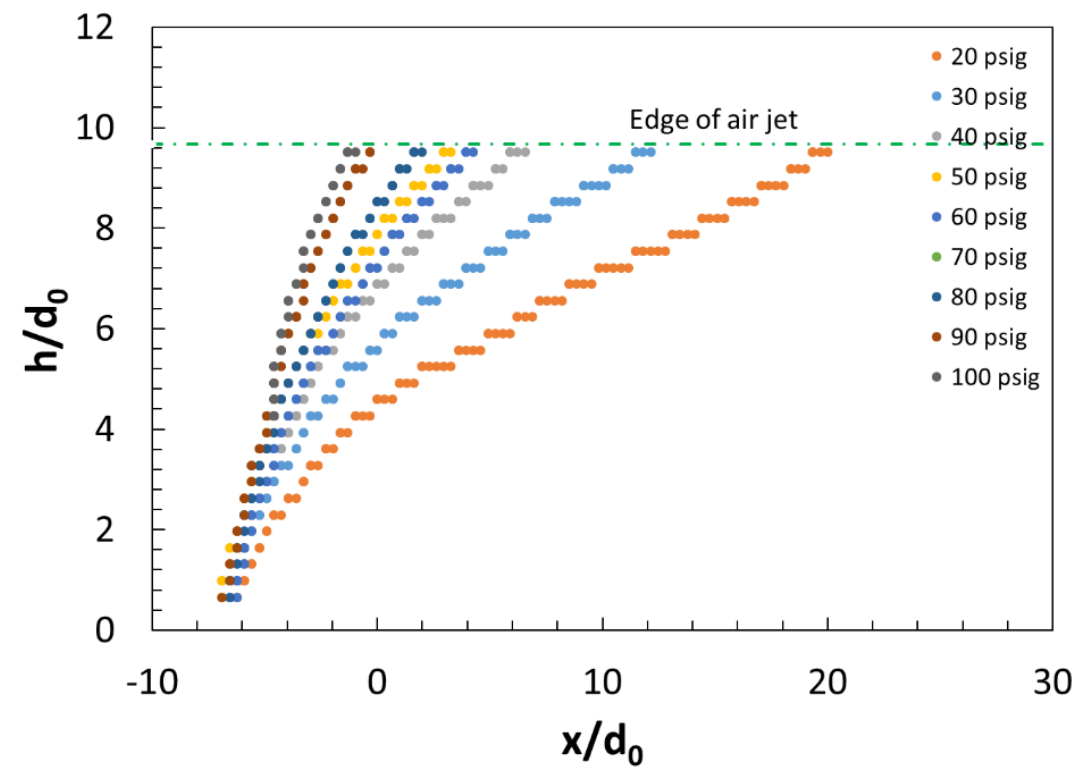

Figure 53. Nozzle 2 air jet penetration

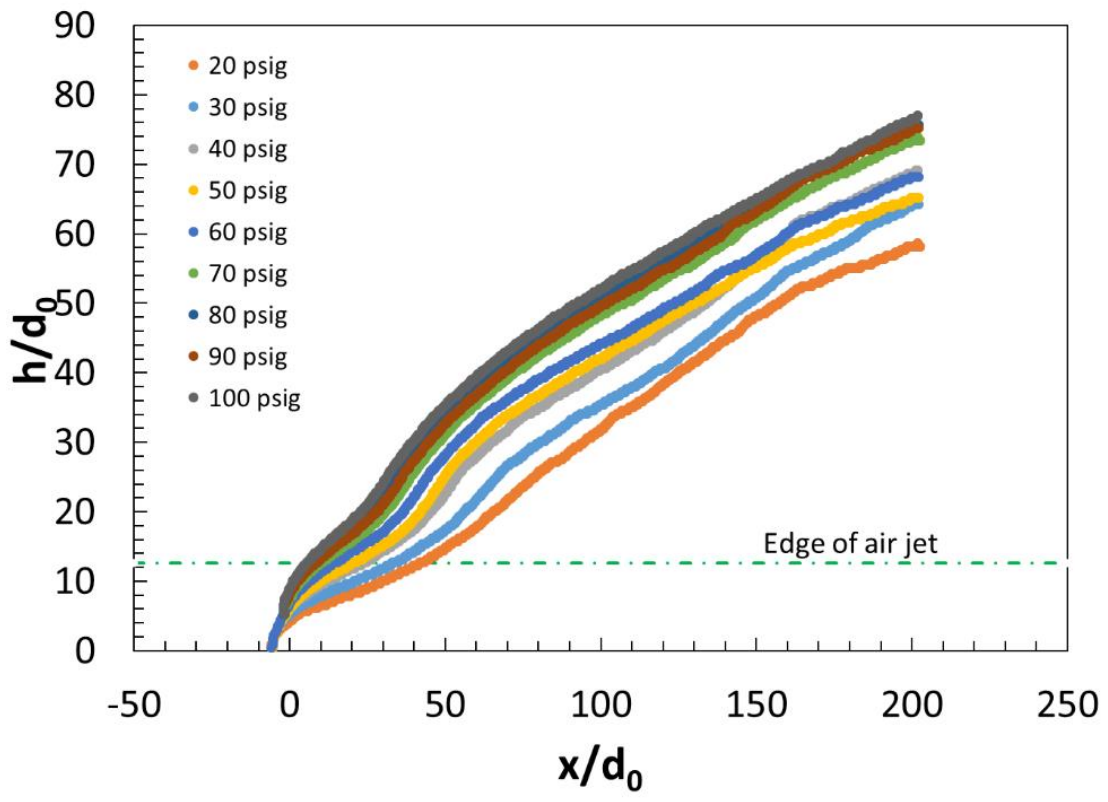

Figure 54. Nozzle 3 global penetration 


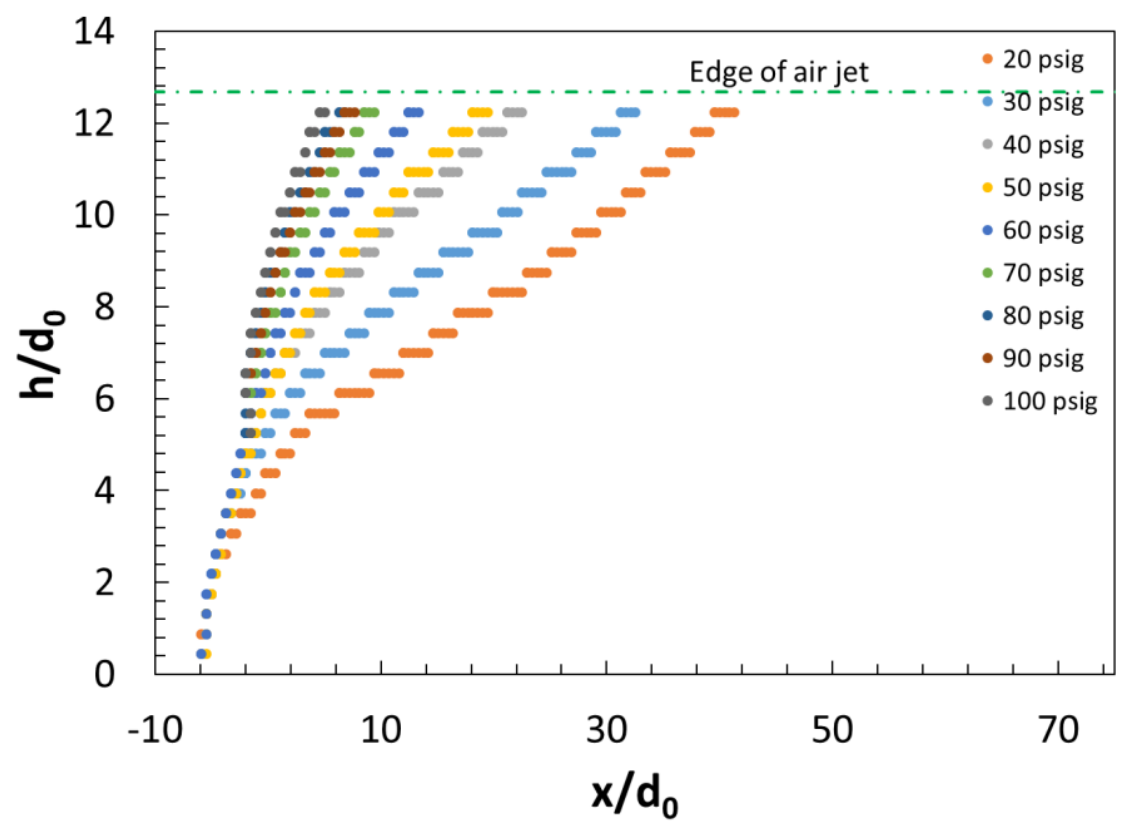

Figure 55. Nozzle 3 air jet penetration

Examining the global penetration data shows increased spreading and global penetration for larger nozzle sizes based on the same dynamic pressure-defined momentum ratio, suggesting a strong influence on nozzle diameter ratio for global penetration. Figure 54 shows a tight spacing of several of the edge locations for increasing momentum ratio for nozzle 3 displaying the evident ability of the air jet to act as a strong sink for the spray momentum at low diameter ratios. Additionally, an increased residence time in the air jet flow region is seen at lower diameter ratios. At low momentum ratios, the liquid flow paths remain within the air flow boundary for a reasonable amount of time and thus, can be compared with traditional correlations for edge penetration from literature. Figure 56 below shows a comparison of liquid jet penetration in the projected gas phase boundary for $\mathrm{q}=0.624$ for all nozzle sizes compared with the predicted penetration from the early correlation developed by Yates [19] along with a secondary comparison to a newer correlation developed by $\mathrm{Wu}$ [20] using more modern PDPA to determine the outer spray boundary location. Both correlations are power law fits in the momentum ratio q. 


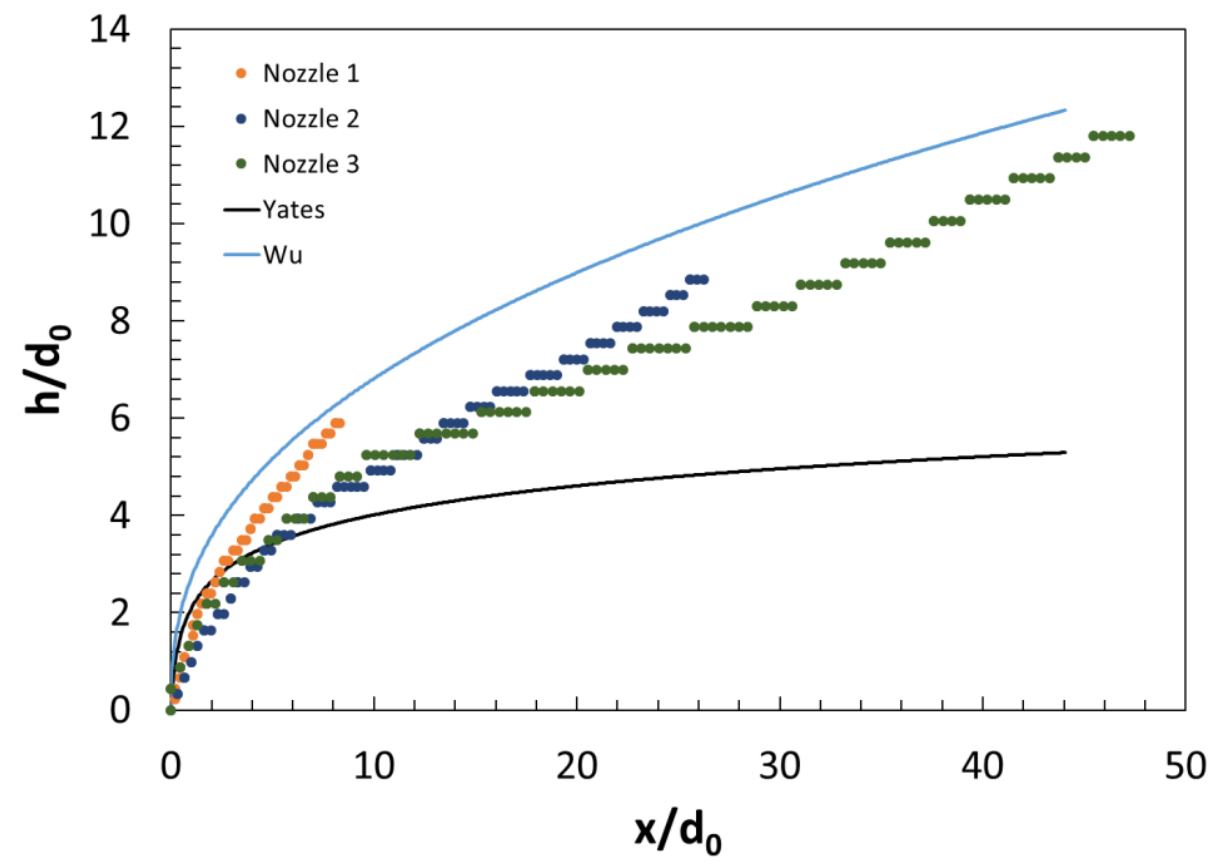

Figure 56. Liquid penetration in projected gas jet location for $q=0.624$ compared with correlation from [19]

The correlation shown above developed by Yates was generated for supersonic crossflows with higher Weber number than the correlation developed by $\mathrm{Wu}$, but as the work by $\mathrm{Wu}$ incorporated laser based diagnostics to locate the spray edge a more exact measure of the spray boundary is obtained; though the results obtained by Wu were for a lower range of Weber number. Equation 4.3 below shows the correlation developed by $\mathrm{Wu}$.

$$
\frac{\bar{y}_{B}}{d_{e}}=3.17 q^{0.33}\left(\frac{\bar{x}}{d_{e}}\right)^{0.04}
$$

As both of these correlations were developed for liquid injection into a continuous crossflow, the deviation from the penetration predicted by the low and high Weber number correlations is thought to be due to flow turning of the gas phase and the low resonance time in the gas phase. Additionally, the correlations were developed for much larger ranges of $\frac{x}{d_{o}}$ and the better 
agreement for the smaller nozzle case where this scale is larger shows the heavy dependence of the agreement to literature on the relative size of the nozzles.

\subsection{Detailed spray structure}

To further quantitatively examine the downstream breakup behavior in the disperse spray downstream of the liquid injection point, PDPA was used to gain statistical information about droplet diameter and velocity at various downstream locations along the gas jet projected centerline. The degree of breakup and ensuing atomization depends critically on the water injection pressure for a fixed air flow. For all the results presented in this work the injection location is maintained the same; at $25.4 \mathrm{~mm}$ below the exit plane of the C-D nozzle and $11 \mathrm{~mm}$ from the centerline of the injector. These dimensions are illustrated in the simulation result shown in

Figure 57. The various locations can also be expressed in terms of the jet diameter as $\bar{x} / d_{e}$, where $\bar{x}$ is the downstream distance from the nozzle exit plane and $d_{e}$ is the effective orifice diameter computed from the jet exit diameter $\left(d_{j}\right) . C_{D}$ is the discharge coefficient of the air nozzle and set equal to 0.7 for this case. The injection location corresponds to 0 jet diameters $\left(\bar{x} / d_{e}=0\right)$ downstream of the liquid injection point. Figure 57 also shows the locations where PDPA measurements were obtained as will be discussed, with Table 5 tabulating these locations along with the injection location $\bar{x} / d_{e}$. 


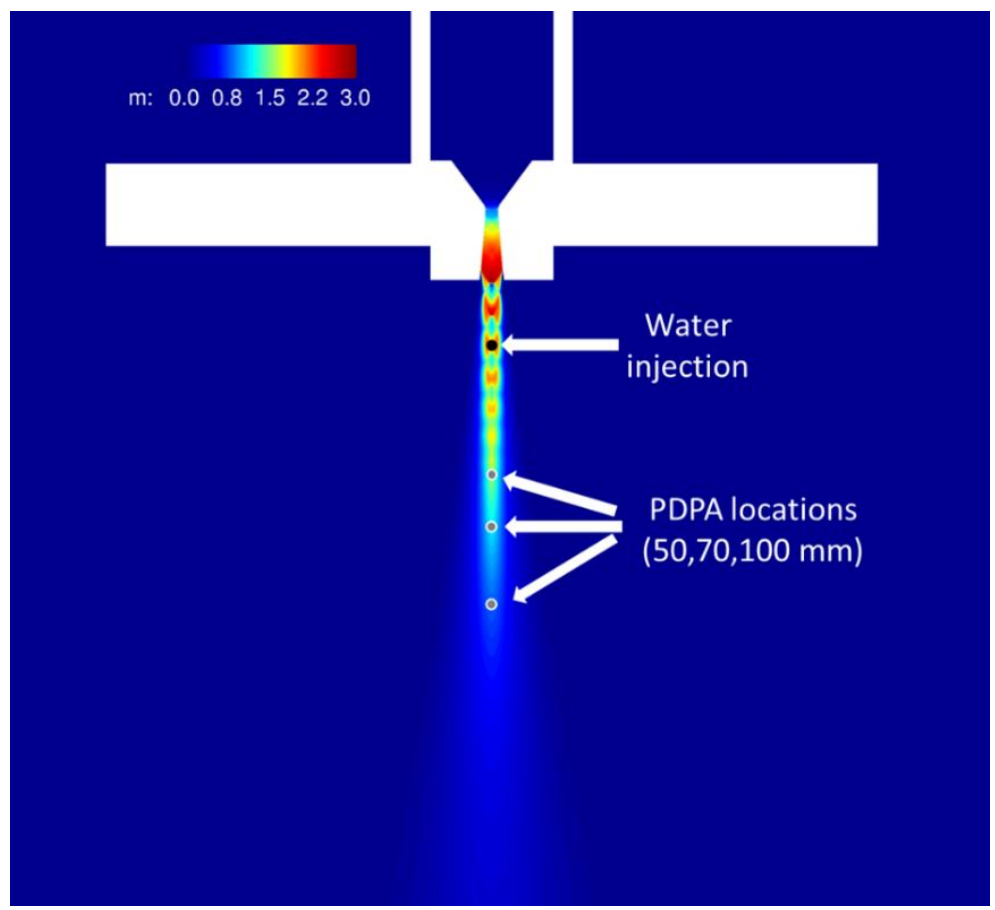

Figure 57. Centerline PDPA measurement locations(Source: Danny Allgood, NASA SSC)

Table 5. Significant locations during PDPA measurements

\begin{tabular}{|c|c|c|}
\hline Location & $\begin{array}{c}\text { Distance from nozzle exit plane } \\
(\mathbf{m m})\end{array}$ & $\overline{\boldsymbol{x}} / \boldsymbol{d}_{\boldsymbol{e}}$ \\
\hline Water injection & 25.4 & 0 \\
\hline PDPA probe location \# 1 & 75.4 & 39 \\
\hline PDPA probe location \# 2 & 95.4 & 55 \\
\hline PDPA probe location \# 3 & 125.4 & 78 \\
\hline
\end{tabular}

The PDPA setup illustrated in Figure 26 was used to obtain quantitative measurements in the water spray. One component velocity (vertical) and droplet size distributions were obtained along the centerline of the air jet. Measurements were obtained at various locations starting from $50 \mathrm{~mm}\left(\bar{x} / d_{e}=39\right)$ below the C-D nozzle exit plane to $100 \mathrm{~mm}\left(\bar{x} / d_{e}=78\right)$ below the exit plane. Figure 58 and Figure 59 below show the results from the PDPA measurements for velocity and droplet size distributions respectively. Results are presented in the form of histograms for three water injection pressures $(20,40$, and $80 \mathrm{psi})$ and at three locations downstream from the C-D nozzle exit plane $(50,70$, and $100 \mathrm{~mm})$. The distributions are primarily of a skewed nature and skewed to the right. 

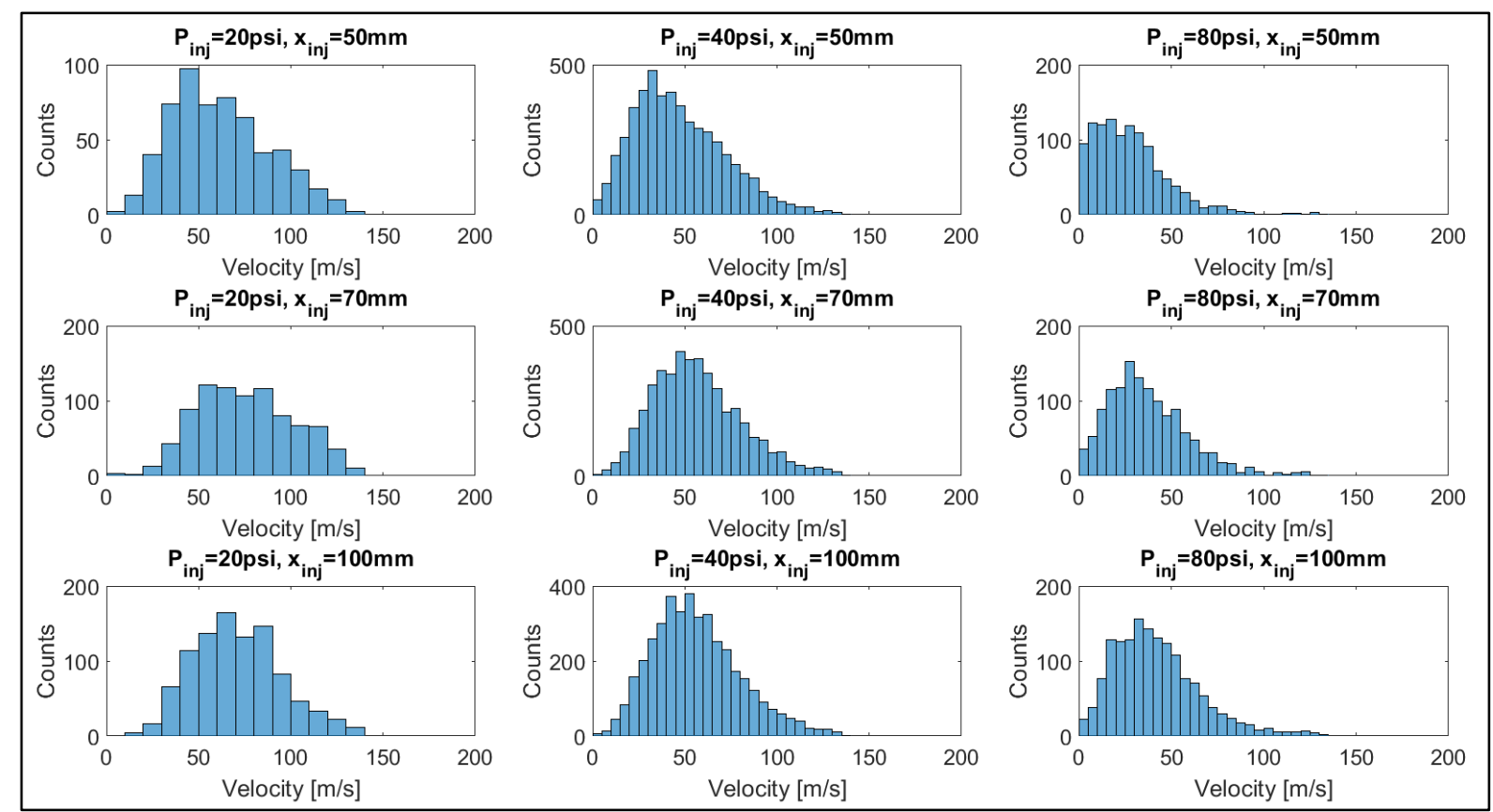

Figure 58. Histograms showing droplet velocity distributions at various injection pressures and locations downstream of the injection location

Figure 58 shows histograms for the downstream component of the droplet velocities with values ranging from 0 to about $150 \mathrm{~m} / \mathrm{s}$ depending on the specific case. Considering the effect of increasing pressure, at a fixed location of $50 \mathrm{~mm}$ (top row of Figure 58), it is observed that while the peak velocity for the 20 psi injection case is around $50 \mathrm{~m} / \mathrm{s}$, the same value shifts to a lower magnitude for the $40 \mathrm{psi}(\sim 30 \mathrm{~m} / \mathrm{s})$ and $80 \mathrm{psi}(\sim 25 \mathrm{~m} / \mathrm{s})$ cases. This is explained by the fact that for the same air flow velocity in all three cases, the air flow must expend more momentum to break up the water jet and turn the spray to make it move in a downward direction. Since the water jet momentum increases with increasing injection pressure, the induced velocity of the water spray droplets decreases for a constant air flow. Additionally, this shift in droplet velocity can be attributed to increased deformation of the mean liquid flow at increased pressure. The liquid stream is turned away from the centerline of the gas jet where measurements are taken, further decreasing the measured velocity as less gas phase momentum is imparted as the flow turns. Considering the same injection pressure but moving to downstream locations, the velocity is observed to increase. 
This is indicated by the histograms shifting towards the right in each column shown in Figure 58. This is caused by the acceleration of the entrained droplets by the air flow given that its momentum is between one to two orders of magnitude greater than that of the water jet as listed in Table 2. The acceleration of the entrained droplets is most evident while comparing the cases for each water injection pressure at 50 and $70 \mathrm{~mm}$ downstream from the exit of the C-D nozzle. From 70 to 100 $\mathrm{mm}$, the acceleration is not as evident likely due to the continuous drop in air flow Mach number as the supersonic jet dissipates energy in the ambient environment resulting in a corresponding decrease in the momentum of the air flow.

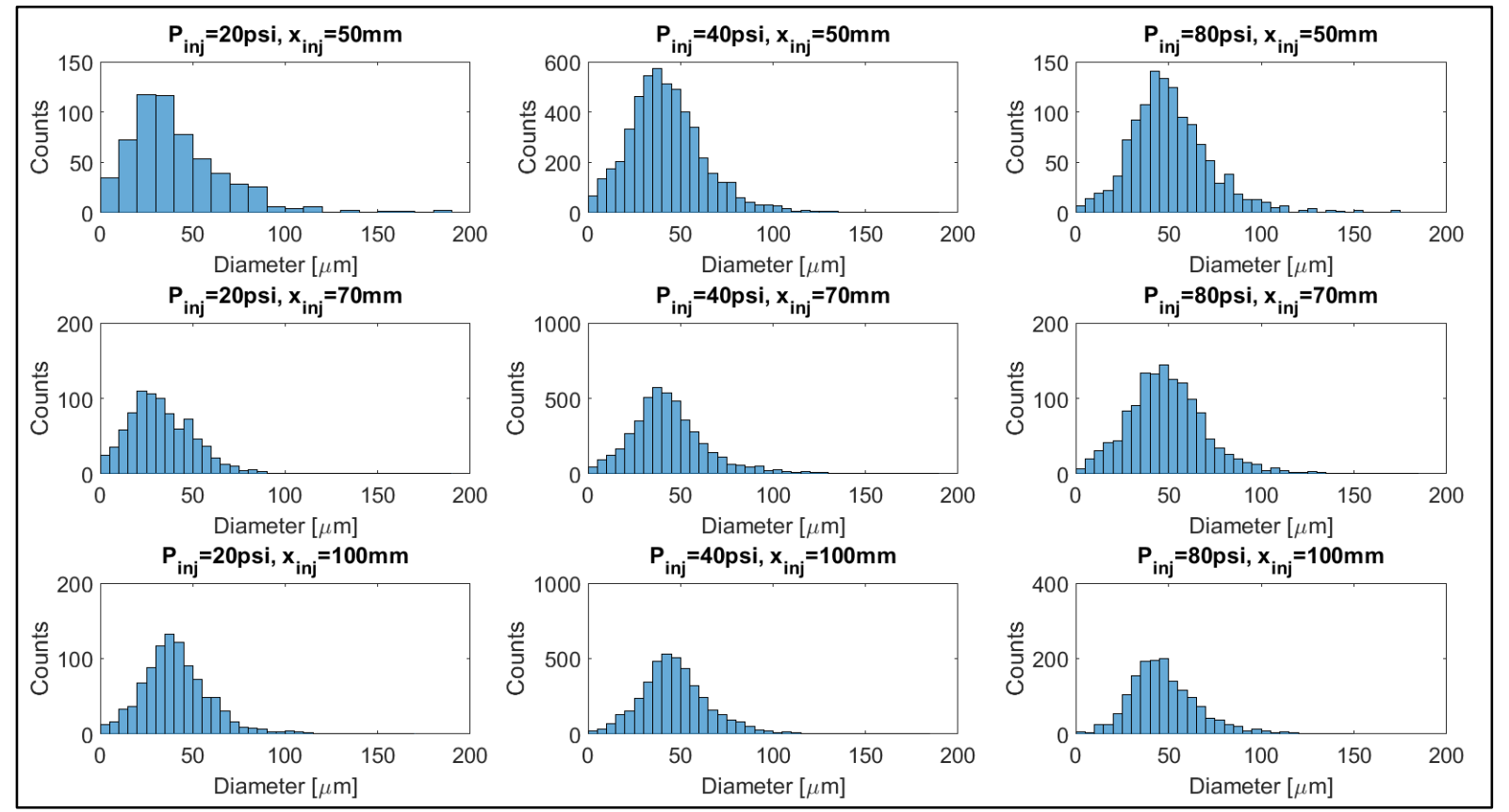

Figure 59. Histograms showing droplet diameter distributions at various injection pressures and locations downstream of the injection location

Considering the droplet size distributions shown in Figure 59, the sizes range from 0 to 100 $\mu \mathrm{m}$ depending on the specific case. As noted in Table 1, the biggest droplet that can be captured using the current PDPA settings has a diameter of about $180 \mu \mathrm{m}$. For the fixed observation location of $50 \mathrm{~mm}$, the effect of increasing the injection pressure is to shift the histogram to the right and 
increase the average size of the droplets. The distribution is also seen to become somewhat narrower. For the large Weber number, low momentum ratio cases investigated in this work, the primary mechanism for the downstream jet breakup is through aerodynamic shattering. As the injection pressure is increased, the higher momentum water jet becomes less susceptible to shattering, resulting in the formation of larger droplets, and thus shifting the distribution of the histograms towards the right. Once the droplets are formed and entrained in the air flow, given the ambient temperatures at which the tests are carried out, there are no significant mechanisms for reducing the size of the droplets such as evaporation. This suggests that the droplet size distribution might stay the same for the same injection pressure as the observation moves to different downstream locations (following a column of pictures in Figure 59). However, the distribution is observed to become somewhat narrower and there is a slight shift in the histograms towards the right. This might be caused by the motion of smaller droplets away from the centerline as the jet expands due to the growth of the shear layer as well as off-axis air flow that may be induced by the outer boundaries of the experiment. Larger droplets having a bigger momentum might be able to stay along the centerline even as smaller droplets are moved out. This can be further investigated using either simulation results or experimental results obtained using PIV.

Figure 60 below shows simulation results for velocity magnitude in the gas phase (left) and simulation results for vertical velocity (right). Due to the conical cross-section of the nozzle and the overexpanded nature of the flow there is a small portion of the gas flow which does not contribute to the axial fluid momentum. The simulation results are for a case with no water injection. Hence it does not account for any induced changes in the air flow structure or dissipation of the supersonic flow caused by the injection of the water jet. Given the finite size of the air jet, it can be assumed that the water injection and ensuing spray formation will result in a considerable 
change in the air jet velocity in the locations where the PDPA measurements were obtained relative to the results shown in Figure 60. However, it is still worthwhile to compare the measured droplet velocity with PDPA to the velocity of the air jet predicted by the simulations in the same spatial location. As can be seen in Figure 60, the vertical gas velocity remains in the range of up to 350 $\mathrm{m} / \mathrm{s}$ near the injection locations for nozzle 1 . At the location where PDPA measurements are obtained the gas velocity varies between $\sim 200-500 \mathrm{~m} / \mathrm{s}$ according to CFD results but is likely less in reality due to flow turning. Comparing this gas velocity with the measured particle velocity at the downstream locations shown in Figure 58 there appears a large discrepancy. As mentioned before, a true estimate can only be made by obtaining an estimate for gas flow velocity downstream of the water jet injection using some experimental or numerical techniques. Besides the disruption in the air jet caused by the water jet injection, additional discrepancies between the droplet velocity and air velocity predicted by Figure 60 could be linked to a large Stokes number Stk for the fluid particles passing through the measurement volume even after secondary breakup of the liquid phase has occurred. In addition, the water injection and subsequent interaction also results some deflection of the air jet resulting in a turning of the gas flow. 


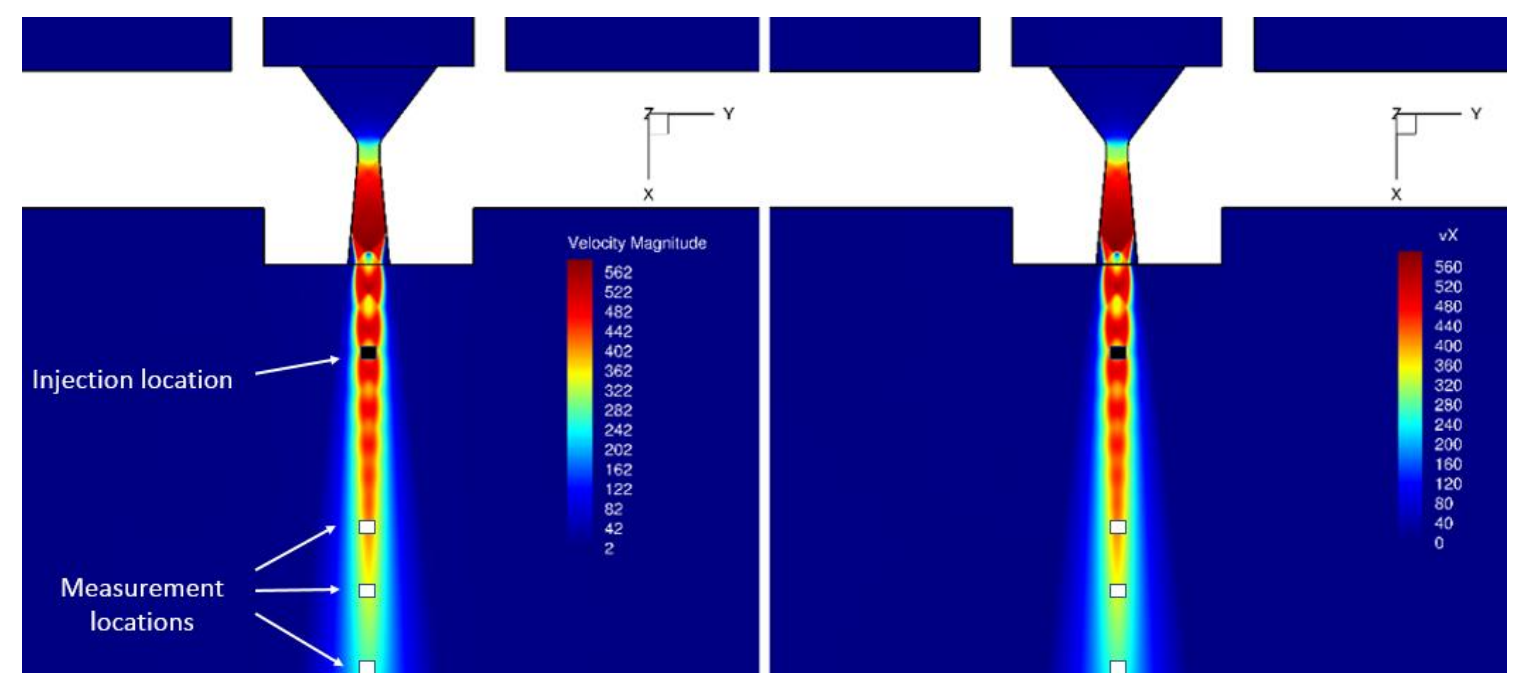

Figure 60. Left: Simulation results for gas phase velocity magnitude, Right: Simulation results for gas phase axial velocity (Source: Danny Allgood, NASA SSC) 


\section{Conclusions and Future Work}

\subsection{Conclusions}

The design challenges involved in the design of water spray rings for suppression of rocket exhaust acoustic and thermal loading on surround structure during testing has necessitated the use of CFD to solve the inherently complex multi-phase engineering problem. To aid in the proper design of these systems, a CFD code has been developed at NASA Stennis Space Center, which has shown good results in large scale testing, but further validation on the small scale is needed. The focus of this work was to examine the general behavior of the interaction of an incompressible liquid jet and a non-combusting supersonic free gas jet with inherent shock structure and draw comparisons to the analogous case of liquid jet in crossflow, a well-established field of study. The qualitative and quantitative measurements performed to characterize this interaction process serves as a direct source of data to validate the compressible, multi-phase CFD code developed at NASA SSC. The supersonic flow in the gas jet and the resulting dense spray upon interaction with the liquid jet necessitated the exclusive use of non-intrusive diagnostics for the measurements performed in this work.

The behavior of the shockwave structure in the gas phase was examined using focusing color schlieren for varied liquid injection velocities and locations. An oblique bow shock is present upstream of the liquid injection point at purely supersonic locations in the gas jet shockwave train. At subsonic locations in the flow slightly downstream of strong shockwaves in the gas phase the inherent shockwave pattern must adjust shape and location to conform to the liquid solid boundary and there is no secondary bow shock as the liquid jet is in a subsonic region. As the jet is injected at a slightly further downstream location where the gas flow is again able to expand to supersonic speed the bow shock reappears as the upstream inherent shock structure has nearly recovered to 
its normal geometry. Liquid jet penetration distance appears to be unaffected by the particular location of injection with reference to the shock train in the overexpanded jet. In contrast to the bounded jet-in-crossflow (JICF) traditionally studied, there is no separation region or shockwave present along the boundary between the potential flow core and the ambient air. Also in contrast to the traditional JICF, the air jet having a finite size with respect to the liquid jet exhibits a broadening due to the blockage by the water jet for a high momentum ratio case. For the low momentum ratio case, the air jet is deflected by the presence of the water jet.

When high-speed volume illuminated photography was used to examine the primary breakup regime of the coherent liquid jet at the injection point with the gas flow, the breakup regime was qualitatively identified to be within the same regime as would be suggested by the traditional regime map used with jet in crossflow. The high speed images reveal jet breakup to occur in a column mode with induced shear causing packets of the liquid jet to be broken off periodically for a low jet momentum ratio case. As the momentum ratio is increased, the breakup mode switches to a surface stripping mode consistent with the trends predicted by the regime diagram. The dynamic behavior of the jet was also explored using proper orthogonal decomposition of a set of snapshots and the major energy containing modes were identified. Further examination of these modes showed flapping and bending modes of the liquid jet consistent with what is expected from literature.

The liquid penetration distance globally and in the projected gas phase boundaries was next examined for varied liquid nozzle diameters and momentum ratios. Ratio of gas to liquid nozzle diameter was found to be an important factor in penetration distance in the global and local regions, even after examining in a normalized reference frame relative to liquid nozzle injection size as is common in literature. For a smaller liquid nozzle size, the penetration in the gas phase qualitatively 
approaches what is expected in literature for traditional JICF. This is expected as the behavior approaches that of liquid jet injected into a supersonic stream of much larger size. Additionally, a characteristic flow feature is identified at a downstream location in the spray as a distinct "hump" in the outer spray edge just past the air jet boundary. This feature is more prominently observed for the cases with the smaller liquid nozzle and lower momentum ratio. This flow feature was identified where there is a possible sudden change in the spray density and could be due to diversion of the gas flow or a sudden atomization phenomenon.

Next, PDPA was used to examine statistical information about droplet size and velocity at several locations along the projected gas phase centerline and thus obtain quantitative data to characterize the spray structure. Droplet sizes and axial velocities were measured at several locations downstream of the injection location. Droplet sizes at a fixed downstream location are found to be directly correlated to the momentum ratio indicating that larger droplets are formed for the cases with high liquid injection pressure where the liquid jet is less susceptible to aerodynamic breakdown by the supersonic air jet. A similar effect results in a shift in the histograms for droplet velocities to lower values with increasing jet momentum ratio. This is caused by the air jet having to expend greater energy to break up the liquid jet resulting in a lower momentum and hence velocity imparted to the spray droplets.

Beyond the characteristic flow features investigated in this work for the interaction of a finite diameter liquid jet with a supersonic free gas jet, the qualitative and quantitative information yielded by the non-intrusive diagnostics provide an extensive set of data that can be used for the validation of the CFD codes for compressible multi-phase flow developed at NASA SSC. Further, the ability to visualize the shock structure in the overexpanded jet and utilize different techniques 
to obtain global and local characteristics of the spray have yielded rich insights into how the liquid jet affects the supersonic free jet and vice versa.

\subsection{Future work}

Future work to better understand the physical processes involved in the liquid breakup should focus on better understanding the effect of liquid to gas diameter ratio, better characterizing the far field breakup pattern for varied injection parameters, and completion of incorporating the hybrid rocket as a means of generating a combusting gas phase flow.

As suggested in literature, alternate forms of the momentum ratio account for a ratio of diameters between the liquid jets as this is an important distinguishing factor when examining free jet interaction compared with liquid jet in crossflow. A larger set of diameter ratios should be examined by choosing both smaller liquid nozzles and larger gas jet nozzles to better span the parameter space to approach a very small liquid to gas jet diameter ratio. The goal of this would be to properly determine if this behavior approaches that traditionally found in jet in crossflow or if there is other physics involved.

Properly measuring downstream breakup physics would necessitate 2D PDPA or PIV to characterize the flow field after liquid injection. The current work only focused on gas centerline locations far downstream and shows a clear need to examine outside this envelope to properly capture the liquid behavior. In addition, a study on the droplet size near the "hump" which was imaged in the current work should be done as it is a clear indication of an important physical process, one that can be replicated by CFD and should be used as validation.

Finally, transition to combusting flow would serve to transition the experiment to a more representative flow regime as is seen on the larger scale test stand. Many of the same diagnostics 
may be used with the exception of FCS, and here phase change of the liquid phase and the corresponding cooling effects on the hot gas can be explored as powerful tools for CFD validation. 


\section{References}

[1] Paur, Jason. "Inside SpaceX's Texas Rocket-Testing Facility." Wired, 27 June 2018, www.wired.com/2012/10/spacex-texas-rocket-test/.

[2] Project Morpheus. (n.d.). Retrieved January 4, 2018, from https://en.wikipedia.org/wiki/Project_Morpheus

[3] Sachdev, J. S., Ahuja, V., Hosangadi, A., and Allgood, D. C., "Analysis of Flame Deflector Spray Nozzles in Rocket Engine Test Stands," 46th AIAA/ASME/SAE/ASEE Joint Propulsion Conference \& Exhibit, Nashville, TN, 2010.

[4] Allgood, D. and Ahuja, V., "Computational plume modeling of conceptual ares vehicle stage tests," 43rd AIAA/ASME/SAE/ASEE Joint Propulsion Conference \& Exhibit, Cincinnati, OH, 2007.

[5] Ferrell, J. (1967). Simulated Space Testing of Propulsion Units and Systems. 4th Space Congress Proceedings.

[6] Yi, J., Yanli, M., Weichen, W., \& Liwu, S. (2010). Inhibition Effect of Water Injection on Afterburning of Rocket Motor Exhaust Plume. Chinese Journal of Aeronautics, 23, 653-659.

[7] Li, J., Jiang, Y., Yu, S., \& Zhou, F. (2015). Cooling Effect of Water Injection on a HighTemperature Supersonic Jet. Energies, 8, 13194-13210.

[8] Risborg, A., \& Soria, J. (2009, February). High-speed optical measurements of an overexpanded su-personic jet impinging on an inclined plate. In 28th international congress on high-speed imaging and photonics (Vol. 7126, p. 71261F). International Society for Optics and Photonics.

[9] Karagozian, A. R. (2014). The jet in crossflow. Physics of Fluids, 26(10), 1-47.

[10] A. Mashayek, and N. Ashgriz, Atomization of a liquid jet in a crossflow, Chap. 29 fin Handbook of Atomization and Sprays (Ashgriz, N. ed.), Springer, 2011, 657-683.

[11] R. Ragucci, A. Bellofiore, and A. Cavaliere, Trajectory and momentum coherence breakdown of a liquid jet in high-density air cross-flow, Atomization and Sprays, 2007, 17, 47-70.

[12] Lin, K. C., Lai, M. C., Ombrello, T., \& Carter, C. D. (2017). Structures and Temporal Evolution of Liquid Jets in Supersonic Crossflow. In 55th AIAA Aerospace Sciences Meeting (p. 1958).

[13] J.N. Stenzler, J.G. Lee, D.A. Santavicca, and W. Lee, Penetration of liquid jets in a crossflow, Atomization and Sprays, 2006,16, 887-906.

[14] C.O. Iyogun, M. Birouk, and N. Popplewell, Trajectory of water jet exposed to low subsonic cross-flow, Atomization and Sprays, 2006, 16, 963-979.

[15] B.J. Masuda, and V.G. McDonell, Penetration of a recessed distillate liquid jet into a crossflow at elevated pressure and temperature, Proc. of 10th Int'l Conf. on Liquid Atomization and Spray Systems, Aug.27-Sept.1, 2006, Kyoto, Japan, Paper ID F4-05275.McDonnel 
[16] Y. Zheng, and A.W. Marshall, Characterization of the initial spray from low- Weber-number jets in crossflow, Atomization and Sprays, 2011, 21(7), 575-589.

[17] No, S. Y. (2015). A review on empirical correlations for jet/spray trajectory of liquid jet in uniform cross flow. International Journal of Spray and Combustion Dynamics, 7(4), 283-313.

[18] Aerospaceweb. Available online: http://www.aerospaceweb.org/question/propulsion/q0224.shtml; (accessed on 14 August 2015).

[19] Yates, C. (1971). Liquid injection into supersonic airstreams. In 7th Propulsion Joint Specialist Conference (p. 724).

[20] P-K. Wu, K.A. Kirkendall, and R.P. Fuller, Spray trajectories of liquid fuel jets in subsonic crossflows, Proceedings of ICLASS-97, Aug.18-22,1998, Seoul, Korea, 545-552.

[21] Sallam, K. A., Lin, K. C., Hammack, S. D., \& Carter, C. D. (2018, July). Effect of Nozzle Geometry on Aerated-Liquid Injection in Supersonic Crossflow. Paper presented at International Conference on Liquid Atomization and Spray Systems, Chicago, IL.

[22] Liu, H., Guo, Y., \& Lin, W. (2016). Numerical simulations of transverse liquid jet to a supersonic crossflow using a pure two-fluid model. Advances in Mechanical Engineering, 8(1), 1687814016629341.

[23] Xiao, F., Dianat, M., \& McGuirk, J. J. (2013). Large eddy simulation of liquid-jet primary breakup in air crossflow. AIAA journal, 51(12), 2878-2893.

[24] Krothapalli, A., Venkatakrishnan, L., Lourenco, L., Greska, B., \& Elavarasan, R. (2003). Turbulence and noise suppression of a high-speed jet by water injection. J. Fluid Mech, 491, 131-159.

[25] Norum, T. D. (n.d.). Reductions in Multi-component Jet Noise by Water Injection. American Institute of Aeronautics and Astronautics.

[26] Greska, B. J. (2005). Supersonic jet noise and its reduction using microjet injection.

[27] Zaman, K., \& Podboy, G. (2010, June). Effect of microjet injection on supersonic jet noise. In 16th AIAA/CEAS Aeroacoustics Conference (p. 4022).

[28] Madabhushi, R. K., Leong, M. Y., \& Arienti, M. (2006, May). ON THE BREAKUP REGIME MAP OF LIQUID JET IN CROSSFLOW. Paper presented at 19th Annual Conference on Liquid Atomization and Spray Systems, Toronto, Canada.

[29] Hybrid rocket engine [drawing]. (2011, November 30). Retrieved from https://www.sciencelearn.org.nz/images/412-hybrid-rocket-engine

[30] Snyder, C. A. (2016, April 2). NASA Chemical Equilibrium with Applications. Retrieved from https://www.grc.nasa.gov/WWW/CEAWeb/

[31] Landis, Geoffrey \& Oleson, Steven \& W. Packard, Thomas \& L. Linne, Diane \& Woytach, Jeffrey \& C. Martini, Michael \& E. Fittje, James \& Z. Gyekenyesi, John \& Colozza, Anthony 
\& Fincannon, James. (2017). Design Study of a Mars Ascent Vehicle for Sample Return Using In-Situ Generated Propellant. 10.2514/6.2017-0424.

[32] Karabeyoglu, A., Zilliac, G., Cantwell, B. J., DeZilwa, S., \& Castellucci, P. (2004). Scale-up tests of high regression rate paraffin-based hybrid rocket fuels. Journal of propulsion and power, 20(6), 1037-1045.

[33] Ostlund, J., \& Muhammad-Klingmann, B. (2005). Supersonic flow separation with application to rocket engine nozzles. Applied Mechanics Reviews, 58(3), 143-177.

[34] Fansler, T. D., \& Parrish, S. E. (2014). Spray measurement technology: a review. Measurement Science and Technology, 26(1), 012002.

[35] Dantec Dynamics A/S. (n.d.). Measurement Principles of PDA. Retrieved from https://www.dantecdynamics.com/measurement-principles-of-pda

[36] Schoegl, I., Pisano, AJ, \& Sedky, G. (2016). Development of a compact focusing color schlieren technique. In 54th AIAA aerospace sciences meeting, San Diego, CA, USA, AIAA (Vol. 1765).

[37] Fillingham, Patrick \& Murali, Harikrishnan \& Novosselov, Igor. (2017). Non-Dimensional Parameter for Characterization of Wall Shear Stress from Underexpanded Axisymmetric Impinging Jets. Journal of Fluids Engineering. 139. 10.1115/1.4037035.

[38] Arienti, M., \& Soteriou, M. C. (2009). Time-resolved proper orthogonal decomposition of liquid jet dynamics. Physics of Fluids, 21(11), 112104.

[39] Berkooz, G., Holmes, P., \& Lumley, J. L. (1993). The proper orthogonal decomposition in the analysis of turbulent flows. Annual review of fluid mechanics, 25(1), 539-575.

[40] Ostlund, J., \& Muhammad-Klingmann, B. (2005). Supersonic flow separation with application to rocket engine nozzles. Applied Mechanics Reviews, 58(3), 143-177.

[41] C. PACK, D. (1950). A note on Prandtl's formula for the wave length of a supersonic jet. Quarterly Journal of Mechanics and Applied Mathematics. 3. 10.1093/qjmam/3.2.173. 


\section{Appendices}

Appendix A- National Instruments DAQ hardware

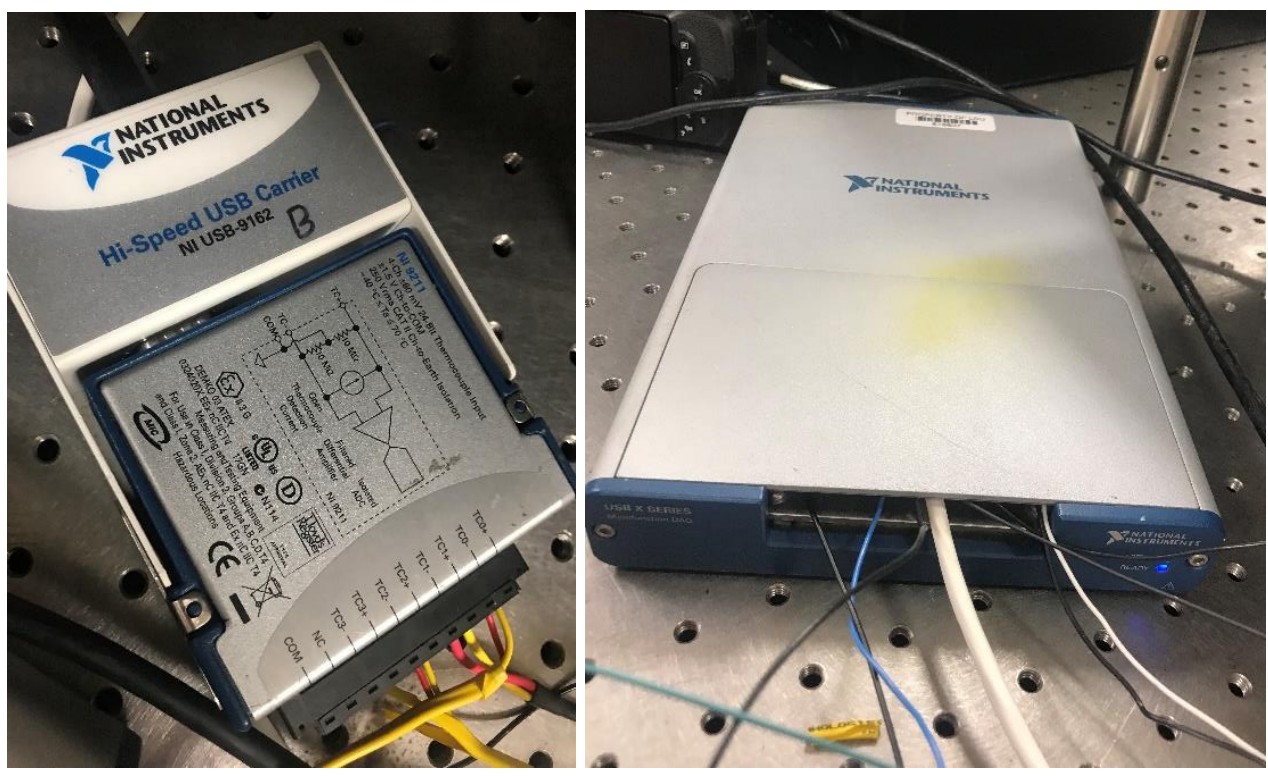

Figure 61. National Instruments DAQ hardware 


\section{Appendix B- PDPA system operating instructions}

\section{D LDA Measurement}

- Connect PC to BSA using the Ethernet port on the front panel of the BSA

If there are issues connecting to the PC, connect the PC to a wired Ethernet port and run the Ethernet diagnostic tool to reset the Ethernet port. Additionally, the Ethernet diagnostic tool can be run without connecting to the internet. This is a known issue when using the Photron high-speed camera as use of the camera requires reassignment of the Ethernet port

- Open the BSA Flow Software and begin a session of 1D LDA measurement and enter the settings shown below from Table 1

\begin{tabular}{|c|c|c|c|}
\hline \multicolumn{2}{|c|}{ Parameter } & Droplet Data & Units \\
\hline \multicolumn{2}{|c|}{ Scattering angle } & 60 & Degrees \\
\hline \multirow{3}{*}{$\begin{array}{l}\text { Probe } \\
\text { volume }\end{array}$} & $\mathrm{x}$-dimension & 0.1943 & $\mathrm{~mm}$ \\
\hline & y-dimension & 0.1941 & $\mathrm{~mm}$ \\
\hline & z-dimension & 4.091 & $\mathrm{~mm}$ \\
\hline \multicolumn{2}{|c|}{ Number of fringes } & 35 & \\
\hline \multicolumn{2}{|c|}{ Fringe spacing } & 5.422 & $\mu \mathrm{m}$ \\
\hline \multicolumn{2}{|c|}{ Beam diameter } & 1.35 & $\mathrm{~mm}$ \\
\hline \multicolumn{2}{|c|}{ Beam separation } & 38 & $\mathrm{~mm}$ \\
\hline \multicolumn{2}{|c|}{ Transmitter focal length } & 400 & $\mathrm{~mm}$ \\
\hline \multicolumn{2}{|c|}{ Receiver focal length } & 600 & $\mathrm{~mm}$ \\
\hline \multicolumn{2}{|c|}{ Maximum particle diameter } & 180 & $\mu \mathrm{m}$ \\
\hline
\end{tabular}

- Ensure a photomultiplier is connected to the "PM1" port on the rear panel of the BSA and the sensing head of the PM is attached to the backscattering probe on the Bragg cell

- Next, to properly align the laser for steady operation of the laser probe, ensure the laser source is properly aligned within the Bragg cell by using the black alignment fixture, this ensures the laser is set at the proper height. Next, set the Bragg cell to the alignment mode and ensure you are wearing laser safety goggles for the remainder of the alignment process. Use the fine 
adjustment wheels on the Bragg cell to align the incident beam and weaker reflected beam onto the center of the alignment window

- Connect the $40 \mathrm{MHz}$ signal output of the BSA to the Bragg cell and ensure the signal output set in the Dantec software is set to this frequency as well

- With the Dantec software running, the relay optics from the Bragg cell must be aligned. Place the laser probe on a fixed surface and use a laser power meter to measure the power of each laser beam being used. Adjust the alignment wheels on the relay optics fixed to the Bragg cell to maximize the power output of all wavelengths of light being used

- Next, with a single wavelength of light, align the probe measurement volume (intersection point of two wavelengths of light being used) to point of interest. To begin taking data, open the measurement pane and press record. Saving data can be done after test is completed

\section{D PDA measurement}

- No adjustments to the laser system need to be made to transition to 1D PDA from 1D LDA measurements. First, the software should be adjusted to begin measuring using 1D PDA. The optical receiver should next have 3 PMs connected to the "U1", "U2", and "U3" slots with each PM connecting to the PM1, PM2, and PM3 connection points on the rear of the BSA, respectively

- After arranging the laser probe and optical receiver at the proper separation distance and angle based on the desired measurement method (refraction, reflection, second order refraction) the optical receiver must be focused on the measurement volume. As this is 1D only one wavelength of light, the one with the most power, should be used; the optical receiver at LSU is set to receive $514.5 \mathrm{~nm}$ green light. Using the humidifier to visualize the measurement 
volume, use the fine adjustment on the optical receiver to focus on the measurement volume (intersection point of laser beams) as tightly as possible and place it on the crosshairs of the receiver

- Return to the Dantec Flow software and open the system monitor pane. As the system is set to 1D PDA, three monitors should be present for each of the 3 PMs used. Using the burst visualization in the system monitor pane, adjust the gain and sensitivity of each PM until the signal strengths are even and the spherical validation and burst validation of the system are maximized

- After closing the system monitor and setting the PM gains and sensitivities, the measurement pane can now be used to record data which may be visualized using a histogram

\section{D PDA measurement}

- First, a second wavelength of light should now be used from the Bragg cell, the one with the highest energy content should be used

- A fourth PM should be connected to the PM4 slot on the rear of the BSA and the sensing head on the PM should be attached to the backscattering probe connected to the Bragg cell. A band pass filter for the specific wavelength of light used for the second velocity component should be placed between the sensing head of the PM and the backscatter probe on the Bragg cell; at LSU there is a filter for the $476.5 \mathrm{~nm}$ (violet) wavelength as this wavelength contains the most energy from the Stabilite continuous laser in use

- After adjusting the software to begin using 2D PDA as the sensing method the same procedures for taking and storing data as well as adjusting the PM sensitivity and gain shown previously may be used 
Incorporating a traverse

- A solid beam should be used to first ensure the laser probe and optical receiver are again in the proper alignment for the measurement technique being used, this solid beam should be rigidly connected to the moving traverse to ensure alignment is kept throughout motion

- The option to add a traverse to the current project in the Dantec software should be chosen by right clicking on the project in the working pane. This will generate a new item in the list for the traverse. After the traverse is connected to the PC via an RS232 cable the traverse and the traverse is powered on, the traverse should be connected to the Dantec software by right clicking on the traverse module

- The traverse(s) can be manually moved using the control command window accessed by right clicking on the traverse module. Each traverse can be moved individually. The number of pulses/in must be set depending on the traverse for movement of the traverses to be accurate; this value can change depending on the model of the traverse being used and is critical if accurate placement of the traverses is important

- A 2D grid of traverse points can also be generated and the traverse can move through each point in the grid after a certain condition on particles measured is met, this can be accessed through by making a movement grid in the traverse module 


\section{Vita}

Hansen was born and raised in Baton Rouge, LA and received his Bachelor of Science in Mechanical Engineering from LSU in May 2016. Following graduation, he worked for 4 months at an aerospace startup in Austin, TX called Firefly Space Systems, and returned to Baton Rouge after the company furloughed its staff in late 2016. He then returned to LSU to begin a Master's degree in January 2017 under the guidance of Dr. Shyam Menon on the first project to come out of the new Energy and Propulsion Laboratory focusing on liquid jet breakup in supersonic free jet crossflows.

Following graduation in December 2018 Hansen will move to Hawthorne, CA to work as a propulsion engineer at Space Exploration Technologies working on propellant management for the company's Falcon 9 launch vehicle. 